for

\title{
Direct electrophilic $\alpha$-fluorination of imines: efficient synthesis of mono- and difluoroimines
}

\author{
Guido Verniest, Eva Van Hende, Riccardo Surmont and Norbert De Kimpe* \\ Department of Organic Chemistry, Faculty of Bioscience Engineering, \\ Ghent University, Coupure Links 653, 9000 Ghent, Belgium
}

TABLE OF CONTENTS:

I. General methods

II. Experimental procedures and spectroscopic data $\left({ }^{1} \mathrm{H}\right.$ NMR, ${ }^{13} \mathrm{C}$ NMR,

${ }^{19} \mathrm{~F}$ NMR, IR, MS, HRMS and elemental analysis) of all new compounds

2e, 2g, 2i, 3a, 3c-g, 5a-c, 6b, 9a-e and 10a-e

III. Copies of ${ }^{1} \mathrm{H}$ NMR and ${ }^{13} \mathrm{C}$ NMR spectra of $\mathbf{2 e}, \mathbf{2 g}, \mathbf{2 i}, \mathbf{3 a - c}, \mathbf{3 f}, \mathbf{3 g}$, 4a-e, 5a-c, 6b, 8a-c, 9a-e, 10a-e

\footnotetext{
* Corresponding author. Tel.: +32 (0)9 26459 51; fax: +32 (0)9 26462 43; e-mail: norbert.dekimpe@UGent.be.
} 


\section{General methods}

${ }^{1} \mathrm{H}$ NMR $(300 \mathrm{MHz}),{ }^{13} \mathrm{C}$ NMR $(75 \mathrm{MHz})$ and ${ }^{19} \mathrm{~F}$ NMR $(282 \mathrm{MHz})$ spectra were run with a Jeol JNM-EX 270 NMR spectrometer or with a Jeol Eclipse FT 300 NMR spectrometer. The compounds were diluted in $\mathrm{CDCl}_{3}$ with tetramethylsilane (TMS, $\delta=0 \mathrm{ppm}$ ) as a reference. ${ }^{19} \mathrm{~F}$ NMR spectra were recorded using $\mathrm{CDCl}_{3}$ as a lock solvent. IR spectra were obtained from a Perkin Elmer Spectrum One infrared spectrometer. Mass spectra were recorded on a Varian MAT 112 spectrometer $(70 \mathrm{eV})$, using either GC-MS coupling or a direct inlet system. Some volatile compounds were recorded on a HP 6890 GC coupled with a HP 5973 MSD (Mass selective detector). LC-MS was performed with an Agilent 1100 Series VS (ES, 4000V) mass spectrometer. HRMS analyses were performed using a Finnigan MAT 95 XP-API-GC Trap tandem mass spectrometer system. Melting points of crystalline compounds were measured with a Büchi 540 apparatus. The elemental analysis of several new compounds was performed on a Perkin Elmer 2400 Elemental Analyser.

\section{Synthetic procedures and spectral data}

\section{Synthesis of imines 2 and 6}

$N$-(1-(4-Chlorophenyl)ethylidene)isopropylamine 2e. The synthesis of imine $2 \mathrm{e}$ is given as a representative example for the synthesis of both imines $\mathbf{2}$ and $\mathbf{6}$ which are used as starting material for further fluorination reactions. Spectral data of known imines $\mathbf{2 a - d}, \mathbf{2} \mathbf{f}$ and $\mathbf{6 a}, \mathbf{b}$ were in accordance with literature data. ${ }^{11}$ To a cooled $\left(0^{\circ} \mathrm{C}\right)$ solution of $10.00 \mathrm{~g}$ of 4 'chloroacetophenone $(64.72 \mathrm{mmol})$ and $15.27 \mathrm{~g}$ of isopropylamine ( $0.26 \mathrm{~mol}, 4$ equiv. $)$ in 250 $\mathrm{ml}$ of dry diethyl ether is added $7.36 \mathrm{~g}$ of titanium(IV) chloride (38.83 mmol, 0.6 equiv.) in 10 $\mathrm{ml}$ of dry pentane. The mixture is stirred for $15 \mathrm{~h}$ allowing the temperature to rise to room temperature. After completion of the reaction, the mixture is poured in a biphasic mixture of $400 \mathrm{ml}$ of aqueous $0.5 \mathrm{M} \mathrm{NaOH}$ solution and $100 \mathrm{ml}$ of diethyl ether. After separation of the organic phase, the aqueous phase is again extracted with $200 \mathrm{ml}$ diethyl ether (2 times). The combined organic phases are dried over a mixture of $\mathrm{MgSO}_{4}$ and $\mathrm{K}_{2} \mathrm{CO}_{3}$ (10:1). Evaporation of the solvent, the crude imine $2 \mathrm{e}$ was distilled. $\mathrm{Bp} 85-90^{\circ} \mathrm{C}(1 \mathrm{mmHg})$, yield $80 \%$. E/Z ratio 92:8. ${ }^{1} \mathrm{H}$ NMR $\left(\mathrm{CDCl}_{3}\right): \delta_{\text {E-isomer }} 1.21\left(6 \mathrm{H}, \mathrm{d}, \mathrm{J}=6.3 \mathrm{~Hz}, 2 \times \mathrm{CH}_{3}\right), 2.22\left(3 \mathrm{H}, \mathrm{s}, \mathrm{CH}_{3}\right), 3.83$ $(1 \mathrm{H}$, sept, $\mathrm{J}=6.3 \mathrm{~Hz}, \mathrm{CHN}), 7.30-7.72(4 \mathrm{H}, \mathrm{m}, 4 \mathrm{x} \mathrm{CH}) . \delta_{\text {Z-isomer }} 1.07(6 \mathrm{H}, \mathrm{d}, \mathrm{J}=6.3 \mathrm{~Hz}, 2 \mathrm{x}$ $\left.\mathrm{CH}_{3}\right), 2.24\left(3 \mathrm{H}, \mathrm{s}, \mathrm{CH}_{3}\right), 3.38(1 \mathrm{H}$, sept, $\mathrm{J}=6.3 \mathrm{~Hz}, \mathrm{CHN}), 7.02-7.40(4 \mathrm{H}, \mathrm{m}, 4 \mathrm{x} \mathrm{CH}) .{ }^{13} \mathrm{C}$ 
NMR $\left(\mathrm{CDCl}_{3}\right)$ : $\delta_{\text {E-isomer }} 14.3\left(\mathrm{CH}_{3}\right), 23.2\left(2 \mathrm{x} \mathrm{CH}_{3}\right), 50.8(\mathrm{CHN}), 127.6\left(2 \times \mathrm{CH}_{\mathrm{ar}}\right), 127.8(2 \mathrm{x}$ $\left.\mathrm{CH}_{\text {ar }}\right), 134.7\left(\mathrm{C}_{\text {quat }}\right), 139.6(\mathrm{CCl}), 160.3(\mathrm{C}=\mathrm{N}) . \delta_{\mathrm{Z} \text {-isomer }} 1.07\left(6 \mathrm{H}, \mathrm{d}, \mathrm{J}=6.3 \mathrm{~Hz}, 2 \times \mathrm{CH}_{3}\right), 2.24$ $\left(3 \mathrm{H}, \mathrm{s}, \mathrm{CH}_{3}\right), 3.38(1 \mathrm{H}$, sept, $\mathrm{J}=\mathrm{CHN}), 7.02-7.40(4 \mathrm{H}, \mathrm{m}, 4 \mathrm{x} \mathrm{CH}) .{ }^{13} \mathrm{C} \mathrm{NMR}\left(\mathrm{CDCl}_{3}\right): \delta_{\mathrm{Z}-}$ isomer $13.9\left(\mathrm{CH}_{3}\right), 23.5\left(2 \times \mathrm{CH}_{3}\right), 51.9(\mathrm{CHN}), 126.8\left(2 \times \mathrm{CH}_{\mathrm{ar}}\right), 128.4\left(2 \times \mathrm{CH}_{\mathrm{ar}}\right), 133.4$ $\left(\mathrm{C}_{\text {quat }}\right), 137.4(\mathrm{CCl}), 164.2(\mathrm{C}=\mathrm{N}) . \mathrm{R}(\mathrm{NaCl}): v_{\max } 1633 \mathrm{~cm}^{-1} . \mathrm{MS}(\mathrm{ES}+) \mathrm{m} / z(\%): 196\left(\mathrm{M}+\mathrm{H}^{+}\right.$, 100). Copies of ${ }^{1} \mathrm{H}$ NMR and ${ }^{13} \mathrm{C}$ NMR spectra are included in chapter III of the supporting information file.

$\mathrm{N}$-((1E)-3,4-Dihydro-1(2H)-naphthalenylidene)isopropylamine $2 \mathrm{~g}$. Bp $133-138^{\circ} \mathrm{C}$ $(11 \mathrm{mmHg})$, yield 79\%. E/Z ratio 100:0. ${ }^{1} \mathrm{H} \mathrm{NMR}\left(\mathrm{CDCl}_{3}\right): \delta 1.20(6 \mathrm{H}, \mathrm{d}, \mathrm{J}=6.2 \mathrm{~Hz}, 2 \mathrm{x}$ $\left.\mathrm{CH}_{3}\right), 1.93\left(2 \mathrm{H}\right.$, quint, $\left.\mathrm{J}=6.3 \mathrm{~Hz}, \mathrm{CH}_{2}\right), 2.60\left(2 \mathrm{H}, \mathrm{t}, \mathrm{J}=6.3 \mathrm{~Hz}, \mathrm{CH}_{2}\right), 2.81(2 \mathrm{H}, \mathrm{t}, \mathrm{J}=6.3 \mathrm{~Hz}$, $\left.\mathrm{CH}_{2}\right), 3.89(1 \mathrm{H}$, sept, $\mathrm{J}=6.2 \mathrm{~Hz}, \mathrm{CHN}), 7.09-7.12(1 \mathrm{H}, \mathrm{m}, \mathrm{CH}), 7.18-7.30(2 \mathrm{H}, \mathrm{m}, 2 \times \mathrm{CH})$, $8.19(1 \mathrm{H}, \mathrm{dd}, \mathrm{J}=7.7 \mathrm{~Hz}, 1.7 \mathrm{~Hz}, \mathrm{CH}) .{ }^{13} \mathrm{C} \mathrm{NMR}\left(\mathrm{CDCl}_{3}\right): \delta 23.1\left(\mathrm{CH}_{2}\right), 23.9\left(2 \mathrm{x} \mathrm{CH}_{3}\right), 27.2$ $\left(\mathrm{CH}_{2}\right), 30.0\left(\mathrm{CH}_{2}\right), 49.9(\mathrm{CHN}), 126.0\left(\mathrm{CH}_{\mathrm{ar}}\right), 126.4\left(\mathrm{CH}_{\mathrm{ar}}\right), 128.4\left(\mathrm{CH}_{\mathrm{ar}}\right), 129.5\left(\mathrm{CH}_{\mathrm{ar}}\right), 135.4$ $\left(\mathrm{C}_{\text {quat }}\right), 140.3\left(\mathrm{C}_{\text {quat }}\right), 161.4(\mathrm{C}=\mathrm{N})$. IR $(\mathrm{NaCl}): v_{\max } 1628,1597,1453,1297 \mathrm{~cm}^{-1} . \mathrm{MS} \mathrm{m} / z(\%)$ : $187\left(\mathrm{M}^{+}, 41\right), 172\left(\mathrm{M}^{+}-\mathrm{CH}_{3}, 100\right), 117$ (43). Copies of ${ }^{1} \mathrm{H}$ NMR and ${ }^{13} \mathrm{C}$ NMR spectra are included in chapter III of the supporting information file.

$N$-(1-Phenylpentylideen)isopropylamine 2 i. $\mathrm{Bp} 80^{\circ} \mathrm{C}(1 \mathrm{mmHg})$, yield $94 \%$. E:Z ratio 59:41 (determined by ${ }^{1} \mathrm{H}$ NMR). ${ }^{1} \mathrm{H}$ NMR $\left(300 \mathrm{MHz}, \mathrm{CDCl}_{3}\right)$ : $\delta_{E \text {-isomer }} 0.90(3 \mathrm{H}, \mathrm{t}$, $\mathrm{J}=7.2 \mathrm{~Hz}, \mathrm{Me}), 1.22(6 \mathrm{H}, \mathrm{d}, \mathrm{J}=6.1 \mathrm{~Hz}, 2 \mathrm{xMe}), 1.25-1.50\left(4 \mathrm{H}, \mathrm{m}, 2 \mathrm{xCH}_{2}\right), 2.64-2.71(2 \mathrm{H}, \mathrm{m}$, $\left.\mathrm{CH}_{2} \mathrm{C}=\mathrm{N}\right), 3.89\left(1 \mathrm{H}\right.$, sept, $\left.\mathrm{J}=6.1 \mathrm{~Hz}, \underline{\mathrm{HMe}}_{2}\right), 7.32-7.42\left(3 \mathrm{H}, \mathrm{m}, 3 \mathrm{xCH}_{\mathrm{ar}}\right), 7.66-7.71(2 \mathrm{H}, \mathrm{m}$, $\left.2 \mathrm{xCH}_{\text {ar,ortho }}\right) . \delta_{Z \text {-isomer }} 0.87(3 \mathrm{H}, \mathrm{t}, \mathrm{J}=7.2 \mathrm{~Hz}, \mathrm{Me}), 1.06(6 \mathrm{H}, \mathrm{d}, \mathrm{J}=6.1 \mathrm{~Hz}, 2 \mathrm{xMe}), 1.25-1.50(4 \mathrm{H}$, $\left.\mathrm{m}, 2 \mathrm{xCH}_{2}\right), 2.46-2.53\left(2 \mathrm{H}, \mathrm{m}, \mathrm{CH}_{2} \mathrm{C}=\mathrm{N}\right), 3.37\left(1 \mathrm{H}\right.$, sept, J=6.1Hz, $\left.\underline{\mathrm{HMe}}_{2}\right), 7.04-7.09(2 \mathrm{H}$, m, $\left.2 \mathrm{xCH}_{\mathrm{ar}, \text { ortho }}\right), 7.32-7.42\left(3 \mathrm{H}, \mathrm{m}, 3 \mathrm{xCH}_{\mathrm{ar}}\right) .{ }^{13} \mathrm{C} \mathrm{NMR}\left(75 \mathrm{MHz}, \mathrm{CDCl}_{3}\right): \delta_{E \text {-isomer }} 13.8(\mathrm{Me})$, $23.0\left(\underline{\mathrm{CH}}_{2} \mathrm{Me}\right), 24.0(2 \mathrm{xMe}), 28.6\left(\underline{\mathrm{CH}}_{2} \mathrm{C}=\mathrm{N}\right), 29.9\left(\underline{\mathrm{CH}}_{2} \mathrm{CH}_{2} \mathrm{Me}\right), 50.7\left(\underline{\mathrm{CHMe}}_{2}\right), 127.0$ (2xCH $\left.\mathrm{xar}_{\text {,ortho }}\right), 128.2\left(2 \mathrm{xCH}_{\mathrm{ar} \text {,meta }}\right), 128.9\left(\mathrm{CH}_{\mathrm{ar}, \text { para }}\right), 141.0\left(\mathrm{C}_{\mathrm{ar}, \text { quat }}\right), 166.5(\mathrm{C}=\mathrm{N}) \cdot \delta_{Z \text {-isomer }} 13.9$ (Me), $22.6\left(\underline{\mathrm{CH}}_{2} \mathrm{Me}\right), 23.9$ (2xMe), $28.7\left(\underline{\mathrm{CH}}_{2} \mathrm{CH}_{2} \mathrm{Me}\right), 42.3\left(\underline{\mathrm{CH}}_{2} \mathrm{C}=\mathrm{N}\right), 52.0\left(\underline{\mathrm{C}} \mathrm{HMe}_{2}\right), 126.2$ (2xCH $\left.\mathrm{xar}_{\text {ortho }}\right), 127.3\left(\mathrm{CH}_{\mathrm{ar}, \text { para }}\right), 128.3\left(2 \mathrm{xCH}_{\text {ar,meta }}\right), 139.1\left(\mathrm{C}_{\mathrm{ar}, \text { quat }}\right), 169.6(\mathrm{C}=\mathrm{N}) . \mathrm{IR}(\mathrm{NaCl})$ : $v_{\max } 1627 \mathrm{~cm}^{-1}$. MS (ES+): m/z(\%): $204\left(\mathrm{M}+\mathrm{H}^{+}, 100\right)$. Copies of ${ }^{1} \mathrm{H}$ NMR and ${ }^{13} \mathrm{C} \mathrm{NMR}$ spectra are included in chapter III of the supporting information file.

$N$-(1-Butylpentylidene)isopropylamine 6b. Bp $45-47^{\circ} \mathrm{C}(0.4 \mathrm{mmHg})$, yield (91\%). ${ }^{1} \mathrm{H}$ NMR $\left(\mathrm{CDCl}_{3}\right): \delta 0.91\left(3 \mathrm{H}, \mathrm{t}, \mathrm{J}=6.9 \mathrm{~Hz}, \mathrm{CH}_{3}\right), 0.93\left(3 \mathrm{H}, \mathrm{t}, \mathrm{J}=6.9 \mathrm{~Hz}, \mathrm{CH}_{3}\right), 1.09(6 \mathrm{H}, \mathrm{d}, \mathrm{J}$ $\left.=6.3 \mathrm{~Hz}, 2 \times \mathrm{CH}_{3}\right), 1.27-1.53\left(8 \mathrm{H}, \mathrm{m}, 4 \times \mathrm{CH}_{2}\right), 2.81\left(2 \mathrm{H}, \mathrm{t}, \mathrm{J}=7.8 \mathrm{~Hz}, \mathrm{CH}_{2 \mathrm{a}} \mathrm{C}=\mathrm{N}\right), 2.82(2 \mathrm{H}$, $\left.\mathrm{t}, \mathrm{J}=7.8 \mathrm{~Hz}, \mathrm{CH}_{2 \mathrm{~b}} \mathrm{C}=\mathrm{N}\right), 3.68(1 \mathrm{H}, \mathrm{sept}, \mathrm{J}=6.3 \mathrm{~Hz}, \mathrm{CHN}) .{ }^{13} \mathrm{C} \mathrm{NMR}\left(\mathrm{CDCl}_{3}\right): \delta 13.8\left(\mathrm{CH}_{3}\right)$, 
$14.0\left(\mathrm{CH}_{3}\right), 22.7\left(\mathrm{CH}_{2}\right), 23.1\left(\mathrm{CH}_{2}\right), 23.9\left(2 \times \mathrm{CH}_{3}\right), 29.4\left(\mathrm{CH}_{2}\right), 29.6\left(\mathrm{CH}_{2}\right), 30.2\left(\mathrm{CH}_{2}\right), 40.3$ $\left(\mathrm{CH}_{2}\right), 49.6(\mathrm{CHN}), 170.4(\mathrm{C}=\mathrm{N})$. IR $(\mathrm{NaCl}): v_{\max } 1655,1466 \mathrm{~cm}^{-1}$. MS m/z $(\%): 183\left(\mathrm{M}^{+}, 2\right)$, 168 (2), 154 (18), 99 (86), 84 (100). Copies of ${ }^{1} \mathrm{H}$ NMR and ${ }^{13} \mathrm{C}$ NMR spectra are included in chapter III of the supporting information file.

\section{2. $\alpha$-Monofluorination of imines 2}

$N$-(2-Fluoro-1-phenylethylidene)isopropylamine 3a. In a flame dried $100 \mathrm{ml}$ flask, a heterogeneous mixture of $2.35 \mathrm{~g} \quad(7.45 \mathrm{mmol}, 1.2$ equiv. $)$ of NFSI $(N-$ fluorobenzenesulfonimide) and $1.72 \mathrm{~g}$ (12.42 mmol, 2 equiv.) of $\mathrm{K}_{2} \mathrm{CO}_{3}$ and $8 \mathrm{~g}$ of $4 \AA$ molecular sieves (dried at $120^{\circ} \mathrm{C}$ ) in $50 \mathrm{ml}$ of acetonitrile and $10 \mathrm{ml}$ of DMF was vigorously stirred under $\mathrm{N}_{2}$ atmosphere for 15 minutes at $0^{\circ} \mathrm{C}$. At the same temperature, $1.00 \mathrm{~g}(6.21$ mmol) of freshly distilled $N$-(1-phenylethylidene)isopropylamine 2a was added dropwise via a syringe. The mixture was stirred for 3 hours at $0^{\circ} \mathrm{C}$. Subsequently, an excess of triethylamine $(5 \mathrm{ml})$ was added at $0^{\circ} \mathrm{C}$ and stirring was continued for 2 minutes. The reaction mixture was filtered over Celite and the solids were washed 3 times with $20 \mathrm{ml}$ of diethyl ether. Subsequently, the filtrate was poured in $100 \mathrm{ml}$ of aqueous $0.5 \mathrm{M} \mathrm{NaOH}$. After separation of the organic layer, the aqueous phase was extracted with $3 \times 25 \mathrm{ml}$ of diethyl ether. To remove the DMF, the combined organic phases were washed with a saturated aqueous NaCl-solution $\left(2 \times 100 \mathrm{ml}\right.$ ) and subsequently dried with $\mathrm{MgSO}_{4} / \mathrm{K}_{2} \mathrm{CO}_{3}$ (10:1). Filtration of the drying agents and evaporation of the solvents in vacuo yielded virtually pure monofluorinated imine 3a (containing only traces of difluorinated imine 9a). Although the crude product is pure enough to be used in further reactions, a further purification could be established by column chromatography (short column) using dry solvents and silica that was dried at high vacuum $(0.01 \mathrm{mmHg})$ at $100^{\circ} \mathrm{C}$ for $5 \mathrm{~h}$ and treated with $\mathrm{Et}_{3} \mathrm{~N}$ directly after cooling to room temperature. Flash chromatography (hexane/EtOAc 9:1, $\mathrm{R}_{\mathrm{f}}=0.37$ ), yield 62\%. E/Z ratio 62:38. ${ }^{1} \mathrm{H} \mathrm{NMR}\left(\mathrm{CDCl}_{3}\right): \delta_{\text {E-isomer }} 1.12\left(6 \mathrm{H}, \mathrm{d}, \mathrm{J}=6.2 \mathrm{~Hz}, 2 \times \mathrm{CH}_{3}\right), 3.57(1 \mathrm{H}$, sept, $\mathrm{J}=6.2 \mathrm{~Hz}, \mathrm{CHN}), 5.06\left(2 \mathrm{H}, \mathrm{d}, \mathrm{J}=47.3 \mathrm{~Hz}, \mathrm{CH}_{2} \mathrm{~F}\right), 7.09-7.90\left(5 \mathrm{H}, \mathrm{m}, \mathrm{C}_{6} \mathrm{H}_{5}\right) . \delta_{\mathrm{Z} \text {-isomer }} 1.25$ $\left(6 \mathrm{H}, \mathrm{d}, \mathrm{J}=6.2 \mathrm{~Hz}, 2 \times \mathrm{CH}_{3}\right), 4.01\left(1 \mathrm{H}\right.$, sept $\left.\mathrm{x} \mathrm{d}, \mathrm{J}_{\mathrm{HH}}=6.2 \mathrm{~Hz}, \mathrm{~J}_{\mathrm{HF}}=1.4 \mathrm{~Hz}, \mathrm{CHN}\right), 5.35(2 \mathrm{H}, \mathrm{d}$, $\left.\mathrm{J}=47.3 \mathrm{~Hz}, \mathrm{CH}_{2} \mathrm{~F}\right), 7.15-7.75\left(5 \mathrm{H}, \mathrm{m}, \mathrm{C}_{6} \mathrm{H}_{5}\right) .{ }^{13} \mathrm{C} \mathrm{NMR}\left(\mathrm{CDCl}_{3}\right): \delta_{\text {E-isomer }} 23.7\left(2 \mathrm{x} \mathrm{CH}_{3}\right), 52.1$ $(\mathrm{CHN}), 85.7\left(\mathrm{~d}, \mathrm{~J}=176.5 \mathrm{~Hz}, \mathrm{CH}_{2} \mathrm{~F}\right), 126.8\left(2 \times \mathrm{CH}_{\mathrm{ar}}\right), 128.6\left(2 \times \mathrm{CH}_{\mathrm{ar}}\right), 128.9\left(\mathrm{CH}_{\mathrm{ar}}\right), 134.69$ $\left(\mathrm{C}_{\text {quat }}\right), 164.0(\mathrm{~d}, \mathrm{~J}=18.5 \mathrm{~Hz}, \mathrm{C}=\mathrm{N}) . \delta_{\mathrm{Z}-\text { isomer }} 24.2\left(2 \mathrm{x} \mathrm{CH}_{3}\right), 51.6(\mathrm{CHN}), 75.7(\mathrm{~d}, \mathrm{~J}=167.3 \mathrm{~Hz}$, $\left.\mathrm{CH}_{2} \mathrm{~F}\right), 127.3\left(2 \times \mathrm{CH}_{\mathrm{ar}}\right), 128.4\left(2 \times \mathrm{CH}_{\mathrm{ar}}\right), 129.8\left(\mathrm{CH}_{\mathrm{ar}}\right), 138.5\left(\mathrm{C}_{\text {quat }}\right), 160.3(\mathrm{~d}, \mathrm{~J}=13.9 \mathrm{~Hz}$, $\mathrm{C}=\mathrm{N}) .{ }^{19} \mathrm{~F} \mathrm{NMR}\left(\mathrm{CDCl}_{3}\right): \delta_{\text {E-isomer }}-220.4\left(\mathrm{t}, \mathrm{J}=47.3 \mathrm{~Hz}, \mathrm{CH}_{2} \mathrm{~F}\right) . \delta_{\text {Z-isomer }}-219.0(\mathrm{t}, \mathrm{J}=47.3 \mathrm{~Hz}$, $\left.\mathrm{CH}_{2} \mathrm{~F}\right)$. IR (NaCl): $v_{\max } 1646 \mathrm{~cm}^{-1}$. MS (ES+) $m / z(\%): 180\left(\mathrm{M}+\mathrm{H}^{+}, 100\right)$. HRMS calcd for 
$\mathrm{C}_{11} \mathrm{H}_{15} \mathrm{NF}\left(\mathrm{M}+\mathrm{H}^{+}\right)$180.11830, found 180.11815. Copies of ${ }^{1} \mathrm{H} \mathrm{NMR}$ and ${ }^{13} \mathrm{C}$ NMR spectra are included in chapter III of the supporting information file.

$\mathrm{N}$-(2-Fluoro-1-phenylpropylidene)isopropylamine $3 \mathbf{b}^{12} \mathrm{Bp} 67-74^{\circ} \mathrm{C}(2.0 \mathrm{mmHg})$, yield $74 \%$. E/Z ratio 97:3. Spectroscopic data were in according to literature data. ${ }^{12}$ HRMS calcd for $\mathrm{C}_{12} \mathrm{H}_{17} \mathrm{NF}\left(\mathrm{M}+\mathrm{H}^{+}\right)$194.13395, found 194.13329. Copies of ${ }^{1} \mathrm{H} \mathrm{NMR}$ and ${ }^{13} \mathrm{C} \mathrm{NMR}$ spectra are included in chapter III of the supporting information file.

$\mathbf{N}$-(2-Fluoro-1-phenylethylidene)butylamine 3c. Not purified, yield 92\% (purity 84\%). E/Z ratio 67:33. ${ }^{1} \mathrm{H}$ NMR $\left(\mathrm{CDCl}_{3}\right): \delta_{\mathrm{E}-\text { isomer }} 0.85\left(3 \mathrm{H}, \mathrm{t}, \mathrm{J}=7.4 \mathrm{~Hz}, \mathrm{CH}_{3}\right), 1.28(2 \mathrm{H}$, sext, $\left.\mathrm{J}=7.4 \mathrm{~Hz}, \mathrm{CH}_{2}\right), 1.60\left(2 \mathrm{H}\right.$, quint $\left., \mathrm{J}=7.4 \mathrm{~Hz}, \mathrm{CH}_{2}\right), 3.33(2 \mathrm{H}, \mathrm{tt}, \mathrm{J}=7.4 \mathrm{~Hz}, 1.1 \mathrm{~Hz}, \mathrm{NCH}), 5.10$ $\left(2 \mathrm{H}, \mathrm{dt}, \mathrm{J}=47.2 \mathrm{~Hz}, 1.1 \mathrm{~Hz}, \mathrm{CH}_{2} \mathrm{~F}\right), 7.17-7.59\left(5 \mathrm{H}, \mathrm{m}, 5 \times \mathrm{CH}_{2}\right) . \delta_{\text {Z-isomer }} 0.97(3 \mathrm{H}, \mathrm{t}, \mathrm{J}=$ 7.4Hz, $\left.\mathrm{CH}_{3}\right), 1.45\left(2 \mathrm{H}\right.$, sext, $\left.\mathrm{J}=7.4 \mathrm{~Hz}, \mathrm{CH}_{2}\right), 1.74\left(2 \mathrm{H}\right.$, quint, $\left.\mathrm{J}=7.4 \mathrm{~Hz}, \mathrm{CH}_{2}\right), 3.64(2 \mathrm{H}, \mathrm{td}$, $\left.\mathrm{J}_{\mathrm{HH}}=7.4 \mathrm{~Hz}, \mathrm{~J}_{\mathrm{HF}}=2.7 \mathrm{~Hz}, \mathrm{NCH}\right), 5.37\left(2 \mathrm{H}, \mathrm{d}, \mathrm{J}=47.2 \mathrm{~Hz}, \mathrm{CH}_{2} \mathrm{~F}\right), 7.17-7.59\left(5 \mathrm{H}, \mathrm{m}, 5 \mathrm{x} \mathrm{CH}_{2}\right)$. ${ }^{13} \mathrm{C} \mathrm{NMR}\left(\mathrm{CDCl}_{3}\right): \delta_{\text {E-isomer }} 24.4\left(2 \times \mathrm{CH}_{2}\right), 25.5\left(\mathrm{CH}_{2}\right), 33.70\left(2 \mathrm{x} \mathrm{CH}_{2}\right), 60.6(\mathrm{NCH}), 85.8(\mathrm{~d}$, $\left.\mathrm{J}=175.4 \mathrm{~Hz}, \mathrm{CH}_{2} \mathrm{~F}\right), 126.9\left(2 \times \mathrm{CH}_{\mathrm{ar}}\right), 128.7\left(2 \times \mathrm{CH}_{\mathrm{ar}}\right), 128.9\left(\mathrm{CH}_{\mathrm{ar}}\right) 134.7\left(\mathrm{C}_{\text {quat }}\right), 164.3(\mathrm{~d}, \mathrm{~J}$ $=18.5 \mathrm{~Hz}, \mathrm{C}=\mathrm{N}) . \delta_{\mathrm{Z} \text {-isomer }} 24.6\left(2 \times \mathrm{CH}_{2}\right), 25.6\left(\mathrm{CH}_{2}\right), 34.1\left(2 \times \mathrm{CH}_{2}\right), 60.1(\mathrm{CHN}), 75.6(\mathrm{~d}, \mathrm{~J}=$ 175.4Hz, $\left.\mathrm{CH}_{2} \mathrm{~F}\right), 127.3\left(2 \times \mathrm{CH}_{\mathrm{ar}}\right), 128.3\left(2 \times \mathrm{CH}_{\mathrm{ar}}\right), 129.7\left(\mathrm{CH}_{\mathrm{ar}}\right), 132.4\left(\mathrm{C}_{\text {quat }}\right), 162.3(\mathrm{~d}, \mathrm{~J}=$ $18.5 \mathrm{~Hz}, \mathrm{C}=\mathrm{N}) .{ }^{19} \mathrm{~F} \mathrm{NMR}\left(\mathrm{CDCl}_{3}\right): \delta_{\mathrm{E} \text {-isomer }}-220.4\left(\mathrm{t}, \mathrm{J}=47.2 \mathrm{~Hz}, \mathrm{CH}_{2} \mathrm{~F}\right) . \delta_{\text {Z-isomer }}-220.7(\mathrm{t}, \mathrm{J}=$ $\left.47.2 \mathrm{~Hz}, \mathrm{CH}_{2} \mathrm{~F}\right)$. IR (NaCl): $v_{\max } 1671 \mathrm{~cm}^{-1}$. MS (ES+) $m / z(\%): 220\left(\mathrm{M}+\mathrm{H}^{+}, 100\right)$. Copies of ${ }^{1} \mathrm{H}$ NMR and ${ }^{13} \mathrm{C}$ NMR spectra are included in chapter III of the supporting information file.

N-(2-Fluoro-1-phenylethylidene)cyclohexylamine 3d. Recrystallization (diethyl ether-petroleum ether), $\mathrm{mp} 56^{\circ} \mathrm{C}$, yield $37 \%$. E/Z ratio 74:26. ${ }^{1} \mathrm{H} \mathrm{NMR}\left(\mathrm{CDCl}_{3}\right)$ : $\delta_{\mathrm{E} \text {-isomer }} 1.02-$ $1.91\left(10 \mathrm{H}, \mathrm{m}, 5 \times \mathrm{CH}_{2}\right), 3.22(1 \mathrm{H}$, quint, $\mathrm{J}=7.4 \mathrm{~Hz}, \mathrm{CHN}), 5.07\left(2 \mathrm{H}, \mathrm{d}, \mathrm{J}=47.3 \mathrm{~Hz}, \mathrm{CH}_{2} \mathrm{~F}\right)$, 7.15-7.20 (2H, m, $\left.2 \times \mathrm{CH}_{\mathrm{ar}}\right), 7.38-7.44\left(3 \mathrm{H}, \mathrm{m}, 3 \mathrm{x} \mathrm{CH}_{\mathrm{ar}}\right) . \delta_{\mathrm{Z} \text {-isomer }} 1.02-191(10 \mathrm{H}, \mathrm{m}, 5 \mathrm{x}$ $\left.\mathrm{CH}_{2}\right), 3.59-3.70(1 \mathrm{H}, \mathrm{m}, \mathrm{CHF}), 5.36\left(2 \mathrm{H}, \mathrm{d}, \mathrm{J}=47.3 \mathrm{~Hz}, \mathrm{CH}_{2} \mathrm{~F}\right), 7.38-7.44\left(3 \mathrm{H}, \mathrm{m}, 3 \mathrm{x} \mathrm{CH} \mathrm{CH}_{\mathrm{ar}}\right)$, 7.72-7.77 (2H, m, $\left.2 \times \mathrm{CH}_{\mathrm{ar}}\right) .{ }^{13} \mathrm{C} \mathrm{NMR}\left(\mathrm{CDCl}_{3}\right)$ : $\delta_{\mathrm{E}-\text { isomer }} 24.4\left(2 \times \mathrm{CH}_{2}\right), 25.5\left(\mathrm{CH}_{2}\right), 33.70(2$ x $\left.\mathrm{CH}_{2}\right), 60.6(\mathrm{NCH}), 85.8\left(\mathrm{~d}, \mathrm{~J}=175.4 \mathrm{~Hz}, \mathrm{CH}_{2} \mathrm{~F}\right), 126.9\left(2 \times \mathrm{CH}_{\mathrm{ar}}\right), 128.7\left(2 \times \mathrm{CH}_{\mathrm{ar}}\right), 128.9$ $\left(\mathrm{CH}_{\mathrm{ar}}\right) 134.7\left(\mathrm{C}_{\text {quat }}\right), 164.3(\mathrm{~d}, \mathrm{~J}=18.5 \mathrm{~Hz}, \mathrm{C}=\mathrm{N}) . \delta_{\mathrm{Z} \text {-isomer }} 24.6\left(2 \mathrm{x} \mathrm{CH}_{2}\right), 25.6\left(\mathrm{CH}_{2}\right), 34.1(2$ x $\left.\mathrm{CH}_{2}\right), 60.1(\mathrm{CHN}), 75.6\left(\mathrm{~d}, \mathrm{~J}=175.4 \mathrm{~Hz}, \mathrm{CH}_{2} \mathrm{~F}\right), 127.3\left(2 \times \mathrm{CH}_{\mathrm{ar}}\right), 128.3\left(2 \times \mathrm{CH}_{\mathrm{ar}}\right), 129.7$ $\left(\mathrm{CH}_{\mathrm{ar}}\right), 132.4\left(\mathrm{C}_{\text {quat }}\right), 162.3(\mathrm{~d}, \mathrm{~J}=18.5 \mathrm{~Hz}, \mathrm{C}=\mathrm{N}) .{ }^{19} \mathrm{~F}$ NMR $\left(\mathrm{CDCl}_{3}\right)$ : $\delta_{\mathrm{E}-\text { isomer }}-220.1(\mathrm{t}, \mathrm{J}=$ $\left.47.3 \mathrm{~Hz}, \mathrm{CH}_{2} \mathrm{~F}\right) . \delta_{\mathrm{Z} \text {-isomer }}-218.6\left(\mathrm{t}, \mathrm{J}=47.3 \mathrm{~Hz}, \mathrm{CH}_{2} \mathrm{~F}\right) . \mathrm{IR}(\mathrm{NaCl}): v_{\max } 1671 \mathrm{~cm}^{-1} . \mathrm{MS}(\mathrm{ES}+)$ $m / z(\%): 220\left(\mathrm{M}+\mathrm{H}^{+}, 100\right)$. Anal. Calcd. for $\mathrm{C}_{14} \mathrm{H}_{18} \mathrm{NF}$ : C, 76.68; H, 8.27; N, 6.39. Found: C, $76.89 ; \mathrm{H}, 8.41 ; \mathrm{N}, 6.26$.

$\mathrm{N}$-(1-(4-Chlorophenyl)-2-fluoroethylidene)isopropylamine

3e. Flash chromatography (hexane/EtOAc 9:1, $\mathrm{R}_{\mathrm{f}}=0.29$ ), yield $68 \%$. E/Z ratio 56:44. ${ }^{1} \mathrm{H}$ NMR 
$\left(\mathrm{CDCl}_{3}\right): \delta_{\text {E-isomer }} 1.11\left(6 \mathrm{H}, \mathrm{d}, \mathrm{J}=6.1 \mathrm{~Hz}, 2 \mathrm{x} \mathrm{CH}_{3}\right), 3.54(1 \mathrm{H}$, sept, $\mathrm{J}=6.1 \mathrm{~Hz}, \mathrm{CHN}), 5.05$ $\left(2 \mathrm{H}, \mathrm{d}, \mathrm{J}=47.3 \mathrm{~Hz}, \mathrm{CH}_{2} \mathrm{~F}\right), 7.12\left(2 \mathrm{H}, \mathrm{d}, \mathrm{J}=8.5 \mathrm{~Hz}, 2 \times \mathrm{CH}_{\mathrm{ar}}\right), 7.42(2 \mathrm{H}, \mathrm{d}, \mathrm{J}=8.5 \mathrm{~Hz}, 2 \mathrm{x}$ $\left.\mathrm{CH}_{\mathrm{ar}}\right) . \delta_{\mathrm{Z} \text {-isomer }} 1.25\left(6 \mathrm{H}, \mathrm{d}, \mathrm{J}=6.1 \mathrm{~Hz}, 2 \mathrm{x} \mathrm{CH}_{3}\right), 3.99\left(1 \mathrm{H}\right.$, sept x d, $\mathrm{J}_{\mathrm{HH}}=6.1 \mathrm{~Hz}, \mathrm{~J}_{\mathrm{HF}}=1.3 \mathrm{~Hz}$, CHN), $5.34\left(2 \mathrm{H}, \mathrm{d}, \mathrm{J}=47.3 \mathrm{~Hz}, \mathrm{CH}_{2} \mathrm{~F}\right), 7.36\left(2 \mathrm{H}, \mathrm{d}, \mathrm{J}=8.8 \mathrm{~Hz}, 2 \times \mathrm{CH}_{\mathrm{ar}}\right), 7.69(2 \mathrm{H}, \mathrm{d}, \mathrm{J}=$ $\left.8.8 \mathrm{~Hz}, 2 \times \mathrm{CH}_{\mathrm{ar}}\right) \cdot{ }^{13} \mathrm{C} \mathrm{NMR}\left(\mathrm{CDCl}_{3}\right): \delta_{\mathrm{E}-\text { isomer }} 23.7\left(2 \times \mathrm{CH}_{3}\right), 52.4(\mathrm{CHN}), 85.7(\mathrm{~d}, \mathrm{~J}=$ 175.4Hz, $\left.\mathrm{CH}_{2} \mathrm{~F}\right), 128.4\left(2 \times \mathrm{CH}_{\mathrm{ar}}\right), 129.0\left(2 \times \mathrm{CH}_{\mathrm{ar}}\right), 133.0\left(\mathrm{C}_{\text {quat }}\right), 135.0(\mathrm{CCl}), 163.0(\mathrm{~d}, \mathrm{~J}=$ 18.4Hz, C=N). $\delta_{\text {Z-isomer }} 24.1\left(2 \times \mathrm{CH}_{3}\right), 51.6(\mathrm{CHN}), 75.3\left(\mathrm{~d}, \mathrm{~J}=170.7 \mathrm{~Hz}, \mathrm{CH}_{2} \mathrm{~F}\right), 128.5(2 \mathrm{x}$ $\left.\mathrm{CH}_{\mathrm{ar}}\right), 128.7\left(2 \times \mathrm{CH}_{\mathrm{ar}}\right), 135.9\left(\mathrm{C}_{\text {quat }}\right), 136.8(\mathrm{CCl}), 159.3(\mathrm{~d}, \mathrm{~J}=15.0 \mathrm{~Hz}, \mathrm{C}=\mathrm{N}) .{ }^{19} \mathrm{~F}$ NMR $\left(\mathrm{CDCl}_{3}\right): \delta_{\text {E-isomer }}-220.5\left(\mathrm{t}, \mathrm{J}=47.3 \mathrm{~Hz}, \mathrm{CH}_{2} \mathrm{~F}\right) . \delta_{\text {Z-isomer }}-219.5\left(\mathrm{t}, \mathrm{J}=47.3 \mathrm{~Hz}, \mathrm{CH}_{2} \mathrm{~F}\right) . \mathrm{IR}$

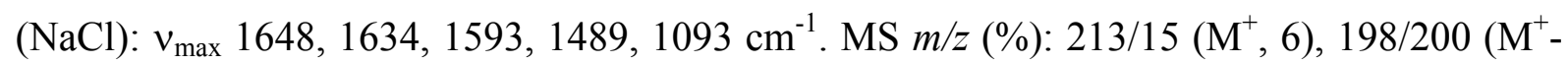
$\left.\mathrm{CH}_{3}, 10\right), 180 / 82$ (30), 138/40(100). Anal. Calcd. for $\mathrm{C}_{11} \mathrm{H}_{13} \mathrm{NClF}$ : C, 61.83; H, 6.13; N, 6.56. Found: C, 61.67; H, 6.30; N, 6.40.

$\mathrm{N}$-(2-Fluoro-1-phenylbutylidene)cyclohexylamine 3f. $\mathrm{Bp} 96^{\circ} \mathrm{C}(0.02 \mathrm{mmHg})$, yield 71\%. E/Z ratio 100:0. ${ }^{1} \mathrm{H}$ NMR $\left(\mathrm{CDCl}_{3}\right): \delta$ 0.91-1.72 $\left(10 \mathrm{H}, \mathrm{m}, 5 \times \mathrm{xCH}_{2}\right), 1.00(3 \mathrm{H}, \mathrm{t}, \mathrm{J}=$ 7.6Hz, $\left.\mathrm{CH}_{3}\right), 1.74-1.84\left(2 \mathrm{H}, \mathrm{m}, \mathrm{CH}_{2}\right), 3.09(1 \mathrm{H}$, quint, $\mathrm{J}=7.3 \mathrm{~Hz}, \mathrm{NCH}), 5.01\left(1 \mathrm{H}, \mathrm{dt}, \mathrm{J}_{\mathrm{HF}}=\right.$ $\left.48.6 \mathrm{~Hz}, \mathrm{~J}_{\mathrm{HH}}=6.3 \mathrm{~Hz}, \mathrm{CHF}\right), 7.10-7.13\left(2 \mathrm{H}, \mathrm{m}, 2 \times \mathrm{CH}_{\mathrm{ar}}\right), 7.33-7.42\left(3 \mathrm{H}, \mathrm{m}, 3 \mathrm{x} \mathrm{CH}_{\mathrm{ar}}\right){ }^{13} \mathrm{C}$ NMR $\left(\mathrm{CDCl}_{3}\right): \delta 9.2\left(\mathrm{~d}, \mathrm{~J}=5.3 \mathrm{~Hz}, \mathrm{CH}_{3}\right), 24.4\left(2 \times \mathrm{CH}_{2}\right), 25.6\left(\mathrm{CH}_{2}\right), 26.0\left(\mathrm{CH}_{2}\right), 26.3\left(\mathrm{CH}_{2}\right)$, $33.8\left(\mathrm{~d}, \mathrm{~J}=13.8 \mathrm{~Hz}, \mathrm{CH}_{2} \mathrm{CHF}\right), 60.7(\mathrm{NCH}), 97.6(\mathrm{~d}, \mathrm{~J}=175.0 \mathrm{~Hz}, \mathrm{CHF}), 127.2\left(2 \times \mathrm{CH}_{\mathrm{ar}}\right)$, $128.4\left(2 \times \mathrm{CH}_{\mathrm{ar}}\right), 128.5\left(\mathrm{CH}_{\mathrm{ar}}\right), 134.8\left(\mathrm{C}_{\text {quat }}\right), 167.0(\mathrm{~d}, \mathrm{~J}=22.3 \mathrm{~Hz}, \mathrm{C}=\mathrm{N}) .{ }^{19} \mathrm{~F} \mathrm{NMR}\left(\mathrm{CDCl}_{3}\right)$ : $\delta-183.8(\mathrm{dt}, \mathrm{J}=48.6 \mathrm{~Hz}, 21.7 \mathrm{~Hz}, \mathrm{CHF}) . \mathrm{IR}(\mathrm{NaCl}): v_{\max } 1647,1449 \mathrm{~cm}^{-1} . \mathrm{MS} \mathrm{m} / z(\%): 247$ $\left(\mathrm{M}^{+}\right.$, 4), 186 (33), 104 (100). HRMS calcd for $\mathrm{C}_{16} \mathrm{H}_{23} \mathrm{NF}\left(\mathrm{M}+\mathrm{H}^{+}\right)$247.17308, found 247.17317. Copies of ${ }^{1} \mathrm{H}$ NMR and ${ }^{13} \mathrm{C}$ NMR spectra are included in chapter III of the supporting information file.

$\mathrm{N}$-((1E)-2-Fluoro-3,4-dihydro-1(2H)-naphthalenylidene)isopropylamine $3 \mathrm{~g} . \quad \mathrm{Bp}$ $94^{\circ} \mathrm{C}(0.3 \mathrm{mmHg})$, yield $71 \% . E / Z$ ratio $0: 100 .{ }^{1} \mathrm{H}$ NMR $\left(\mathrm{CDCl}_{3}\right): \delta 1.22(3 \mathrm{H}, \mathrm{d}, \mathrm{J}=6.2 \mathrm{~Hz}$, $\left.\mathrm{CH}_{3}\right), 1.28\left(3 \mathrm{H}, \mathrm{d}, \mathrm{J}=6.2 \mathrm{~Hz}, \mathrm{CH}_{3}\right), 1.87-2.12\left(1 \mathrm{H}, \mathrm{m}, \mathrm{C} H_{a} \mathrm{H}_{\mathrm{b}} \mathrm{CHF}\right), 2.38-2.51(1 \mathrm{H}, \mathrm{m}$, $\left.\mathrm{CH}_{\mathrm{a}} \mathrm{H}_{b} \mathrm{CHF}\right), 2.71\left(1 \mathrm{H}, \mathrm{dt}, \mathrm{J}=15.8 \mathrm{~Hz}, 4.1 \mathrm{~Hz}, \mathrm{CH}_{a} \mathrm{H}_{\mathrm{b}} \mathrm{CH}_{2} \mathrm{CHF}\right), 3.09$ ( $1 \mathrm{H}$, ddd, J=15.8Hz, $\left.12.1 \mathrm{~Hz}, 4.1 \mathrm{~Hz}, \mathrm{CH}_{\mathrm{a}} \mathrm{H}_{b} \mathrm{CH}_{2} \mathrm{CHF}\right), 4.19\left(1 \mathrm{H}\right.$, sept x d, $\left.\mathrm{J}_{\mathrm{HH}}=6.2 \mathrm{~Hz}, \mathrm{~J}_{\mathrm{HF}}=2.4 \mathrm{~Hz}, \mathrm{CHN}\right), 5.69$ $\left(1 \mathrm{H}, \mathrm{dt}, \mathrm{J}_{\mathrm{HF}}=43.8 \mathrm{~Hz}, \mathrm{~J}_{\mathrm{HH}}=3.5 \mathrm{~Hz}, \mathrm{CHF}\right), 7.14\left(1 \mathrm{H}, \mathrm{d}, \mathrm{J}=7.4 \mathrm{~Hz}, \mathrm{CH}_{\mathrm{ar}}\right), 7.22-7.33(2 \mathrm{H}, \mathrm{m}, 2$ $\mathrm{x} \mathrm{CH}$ ar $), 8.18\left(1 \mathrm{H}, \mathrm{dd}, 7.6 \mathrm{~Hz}, 1.5 \mathrm{~Hz}, \mathrm{CH}_{\mathrm{ar}}\right) .{ }^{13} \mathrm{C} \mathrm{NMR}\left(\mathrm{CDCl}_{3}\right): \delta 23.9\left(\mathrm{~d}, \mathrm{~J}=4.6 \mathrm{~Hz}, \mathrm{CH}_{2}\right)$, $24.4\left(\mathrm{CH}_{3}\right), 24.5\left(\mathrm{CH}_{3}\right), 29.5\left(\mathrm{~d}, \mathrm{~J}=20.8 \mathrm{~Hz}, \mathrm{CH}_{2}\right), 51.1(\mathrm{NCH}), 81.2(\mathrm{~d}, \mathrm{~J}=171.9 \mathrm{~Hz}, \mathrm{CHF})$, 126.6 $\left(\mathrm{CH}_{\mathrm{ar}}\right), 126.8\left(\mathrm{CH}_{\mathrm{ar}}\right), 128.2\left(\mathrm{CH}_{\mathrm{ar}}\right), 130.0\left(\mathrm{CH}_{\mathrm{ar}}\right), 133.4\left(\mathrm{C}_{\text {quat }}\right), 139.4\left(\mathrm{C}_{\text {quat }}\right), 156.5(\mathrm{~d}, \mathrm{~J}$ $=13.8 \mathrm{~Hz}, \mathrm{C}=\mathrm{N}) .{ }^{19} \mathrm{~F}$ NMR $\left(\mathrm{CDCl}_{3}\right): \delta-183.2(\mathrm{dt}, \mathrm{J}=43.8 \mathrm{~Hz}, 7.0 \mathrm{~Hz}, \mathrm{CHF}) . \mathrm{IR}(\mathrm{NaCl}): v_{\max }$ 1629, 1599, $1455 \mathrm{~cm}^{-1}$. MS m/z (\%): $205\left(\mathrm{M}^{+}, 18\right), 190$ (77), 117 (100). HRMS calcd for 
$\mathrm{C}_{13} \mathrm{H}_{17} \mathrm{NF}\left(\mathrm{M}+\mathrm{H}^{+}\right)$206.13395, found 206.13411. Copies of ${ }^{1} \mathrm{H} \mathrm{NMR}$ and ${ }^{13} \mathrm{C} \mathrm{NMR}$ spectra are included in chapter III of the supporting information file.

\section{Synthesis of $\alpha$-fluoroketones 4}

2-Fluoroacetophenone $4 \mathbf{a}^{14}$ To a solution of 0.5 g of $N$-(2-fluoro-1phenylethylidene)isopropylamine 3a in $20 \mathrm{ml}$ of dichloromethane was added $20 \mathrm{ml}$ of aqueous $2 \mathrm{M} \mathrm{HCl}$. The mixture was stirred at room temperature for 2 hours and subsequently, the organic layer was separated. After extraction of the aqueous phase with $\mathrm{CH}_{2} \mathrm{Cl}_{2}(2 \times 25$ $\mathrm{ml})$, the organic phase was dried $\left(\mathrm{MgSO}_{4}\right)$, filtered and the solvent evaporated, yielding almost pure 2-fluoroacetophenone 4a. A further purification was possible via chromatography over a very short plug of silica gel. Flash chromatography (hexane/EtOAc 9:1, $\mathrm{R}_{\mathrm{f}}=0.52$ ) yielded $91 \%$ of 2 -fluoroacetophenone. Spectral data were in accordance with literature data. ${ }^{14}$ Copies of ${ }^{1} \mathrm{H}$ NMR and ${ }^{13} \mathrm{C}$ NMR spectra as an indication of purity are included in chapter III of the supporting information file.

2-Fluoropropiophenone $4 \mathbf{b} .^{8}$ Flash chromatography (hexane/EtOAc 9:1, $\mathrm{R}_{\mathrm{f}}=0.39$ ), yield $85 \%$. Spectral data were in accordance with literature data. ${ }^{8}$ Copies of ${ }^{1} \mathrm{H}$ NMR and ${ }^{13} \mathrm{C}$ NMR spectra as an indication of purity are included in chapter III of the supporting information file.

1-(4-Chlorophenyl)-2-fluoroethanone $4 c{ }^{14}$ Flash chromatography (hexane/EtOAc 9:1, $\mathrm{R}_{\mathrm{f}}=0.53$ ), yield $94 \%$. Spectral data were in accordance with literature data. ${ }^{14}$ Copies of ${ }^{1} \mathrm{H}$ NMR and ${ }^{13} \mathrm{C}$ NMR spectra as an indication of purity are included in chapter III of the supporting information file.

2-Fluorobutyrophenone 4d. ${ }^{15}$ Flash chromatography (hexane/EtOAc 9:1, $\mathrm{R}_{\mathrm{f}}=0.40$ ), yield $90 \%$. Spectral data were in accordance with literature data. ${ }^{15}$ Copies of ${ }^{1} \mathrm{H}$ NMR and ${ }^{13} \mathrm{C}$ NMR spectra as an indication of purity are included in chapter III of the supporting information file.

2-Fluoro- $\alpha$-tetralone 4e. ${ }^{6 \mathrm{i}}$ Flash chromatography (hexane/EtOAc 9:1, $\mathrm{R}_{\mathrm{f}}=0.43$ ), yield $86 \%$. Spectral data were in accordance with literature data. ${ }^{6 \mathrm{i}}$ Copies of ${ }^{1} \mathrm{H}$ NMR and ${ }^{13} \mathrm{C}$ NMR spectra as an indication of purity are included in chapter III of the supporting information file.

\section{Synthesis of $\beta$-fluorinated amines 5}

$\boldsymbol{N}$-(2-Fluoro-1-phenylethyl)- $\boldsymbol{N}$-isopropylamine 5a. To a solution of $0.5 \mathrm{~g}$ (2.79 mmol) of imine 3a in $25 \mathrm{ml}$ of absolute methanol was added $0.19 \mathrm{~g}$ (3.07 mmol, 1.1 equiv.) of 
acetic acid and $0.18 \mathrm{~g}\left(3.07 \mathrm{mmol}, 1.1\right.$ equiv.) of sodium cyanoborohydride at $0^{\circ} \mathrm{C}$. The mixture was stirred for $15 \mathrm{~h}$ while the temperature reached room temperature. After completion of the reaction, the mixture was poured in $50 \mathrm{ml}$ of a saturated aqueous $\mathrm{NaHCO}_{3}$ solution and was subsequently extracted with diethyl ether $(3 \times 50 \mathrm{ml})$. The combined organic layers were washed with brine and dried over $\mathrm{MgSO}_{4}$. Filtration of the solids and evaporation of the solvent yielded almost pure $N$-(2-fluoro-1-phenylethyl)- $N$-isopropylamine 5a which could be further purified by flash chromatography (hexane/EtOAc 98:2, $\mathrm{R}_{\mathrm{f}}=0.08$ ), yield 72\%. ${ }^{1} \mathrm{H}$ NMR $\left(\mathrm{CDCl}_{3}\right): \delta 1.01(3 \mathrm{H}, \mathrm{d}, \mathrm{J}=6.3 \mathrm{~Hz}, \mathrm{Me}), 1.04(3 \mathrm{H}, \mathrm{d}, \mathrm{J}=6.3 \mathrm{~Hz}, \mathrm{Me}), 2.69$ $(1 \mathrm{H}$, sept, $\mathrm{J}=6.3 \mathrm{~Hz}, \mathrm{NCH}), 4.11\left(1 \mathrm{H}, \mathrm{ddd}, \mathrm{J}_{\mathrm{HF}}=12.3 \mathrm{~Hz}, \mathrm{~J}_{\mathrm{HH}}=7.8 \mathrm{~Hz}, \mathrm{~J}_{\mathrm{HH}}=4.2 \mathrm{~Hz}, \mathrm{NCH}\right)$, $4.35\left(1 \mathrm{H}, \mathrm{ddd}, \mathrm{J}_{\mathrm{HF}}=47.3 \mathrm{~Hz}, \mathrm{~J}_{\mathrm{HH}}=8.9 \mathrm{~Hz}, \mathrm{~J}_{\mathrm{HH}}=7.8 \mathrm{~Hz}, \mathrm{C}(\mathrm{H}) H \mathrm{~F}\right), 4.48\left(1 \mathrm{H}, \mathrm{ddd}, \mathrm{J}_{\mathrm{HF}}=47.3 \mathrm{~Hz}\right.$, $\left.\mathrm{J}_{\mathrm{HH}}=8.9 \mathrm{~Hz}, \mathrm{~J}_{\mathrm{HH}}=4.2 \mathrm{~Hz}, \mathrm{C}(H) \mathrm{HF}\right), 7.01-7.63\left(5 \mathrm{H}, \mathrm{m}, 5 \mathrm{x} \mathrm{CH} \mathrm{Cr}_{\mathrm{ar}}\right){ }^{13} \mathrm{C} \mathrm{NMR}\left(\mathrm{CDCl}_{3}\right): \delta 22.2$ $\left(\mathrm{CH}_{3}\right), 24.3\left(\mathrm{CH}_{3}\right), 45.9(\mathrm{NCH}), 60.2(\mathrm{~d}, \mathrm{~J}=18.5 \mathrm{~Hz}, \mathrm{NCH}), 87.2\left(\mathrm{~d}, \mathrm{~J}=175.4 \mathrm{~Hz}, \mathrm{CH}_{2} \mathrm{~F}\right)$, $127.8\left(2 \times \mathrm{CH}_{\mathrm{ar}}\right), 127.9\left(\mathrm{CH}_{\mathrm{ar}}\right), 128.7\left(2 \times \mathrm{CH}_{\mathrm{ar}}\right), 139.4\left(\mathrm{~d}, \mathrm{~J}=6.9 \mathrm{~Hz}, \mathrm{C}_{\text {quat }}\right) .{ }^{19} \mathrm{~F} \mathrm{NMR}$ $\left(\mathrm{CDCl}_{3}\right): \delta-219.3(\mathrm{dt}, \mathrm{J}=12.3 \mathrm{~Hz}, 47.3 \mathrm{~Hz}, \mathrm{CHF})$. IR $(\mathrm{NaCl}): v_{\max } 3331 \mathrm{~cm}^{-1}$. MS (ES+) $\mathrm{m} / \mathrm{z}$ (\%): $182\left(\mathrm{M}+\mathrm{H}^{+}, 100\right)$. HRMS calcd for $\mathrm{C}_{11} \mathrm{H}_{17} \mathrm{NF}\left(\mathrm{M}+\mathrm{H}^{+}\right)$182.13395, found 182.13422. Copies of ${ }^{1} \mathrm{H}$ NMR and ${ }^{13} \mathrm{C}$ NMR spectra are included in chapter III of the supporting information file.

$\boldsymbol{N}$-(2-Fluoro-1-(4-chlorophenyl)ethyl)- $\mathbf{N}$-isopropylamine 5b. Flash chromatography (hexane/EtOAc/Et $3 \mathrm{~N}$ 95:4.9:0.1, $\left.\mathrm{R}_{\mathrm{f}}=0.16\right)$, yield 68\%. ${ }^{1} \mathrm{H}$ NMR $\left(\mathrm{CDCl}_{3}\right): \delta 1.00(3 \mathrm{H}, \mathrm{d}, \mathrm{J}=$ 6.3Hz, Me), $1.04(3 \mathrm{H}, \mathrm{d}, \mathrm{J}=6.3 \mathrm{~Hz}, \mathrm{Me}), 1.5(1 \mathrm{H}, \mathrm{s}(\mathrm{b}), \mathrm{NH}), 2.66(1 \mathrm{H}, \mathrm{sept}, \mathrm{J}=6.3 \mathrm{~Hz}, \mathrm{NCH})$, $4.09\left(1 \mathrm{H}, \mathrm{ddd}, \mathrm{J}_{\mathrm{HF}}=12.6 \mathrm{~Hz}, \mathrm{~J}_{\mathrm{HH}}=7.7 \mathrm{~Hz}, \mathrm{~J}_{\mathrm{HH}}=4.3 \mathrm{~Hz}, \mathrm{NCH}\right), 4.32\left(1 \mathrm{H}, \mathrm{ddd}, \mathrm{J}_{\mathrm{HF}}=46.9 \mathrm{~Hz}\right.$, $\left.\mathrm{J}_{\mathrm{HH}}=8.9 \mathrm{~Hz}, \mathrm{~J}_{\mathrm{HH}}=7.7 \mathrm{~Hz}, \mathrm{C}(\mathrm{H}) H \mathrm{~F}\right), 4.42\left(1 \mathrm{H}, \mathrm{ddd}, \mathrm{J}_{\mathrm{HF}}=46.9 \mathrm{~Hz}, \mathrm{~J}_{\mathrm{HH}}=8.9 \mathrm{~Hz}, \mathrm{~J}_{\mathrm{HH}}=4.3 \mathrm{~Hz}\right.$, $\mathrm{C}(H) \mathrm{HF}), 7.31\left(4 \mathrm{H}, \mathrm{s}, 4 \times \mathrm{CH}_{\mathrm{ar}}\right) .{ }^{13} \mathrm{C} \mathrm{NMR}\left(\mathrm{CDCl}_{3}\right): \delta 22.1\left(\mathrm{CH}_{3}\right), 24.3\left(\mathrm{CH}_{3}\right), 45.9(\mathrm{NCH})$, $59.7(\mathrm{~d}, \mathrm{~J}=18.5 \mathrm{~Hz}, \mathrm{NCH}), 86.9\left(\mathrm{~d}, \mathrm{~J}=175.4 \mathrm{~Hz}, \mathrm{CH}_{2} \mathrm{~F}\right), 128.8\left(2 \times \mathrm{CH}_{\mathrm{ar}}\right), 129.1\left(2 \times \mathrm{CH}_{\mathrm{ar}}\right)$, $133.5\left(\mathrm{C}_{\text {quat }}\right), 138.1\left(\mathrm{~d}, \mathrm{~J}=6.9 \mathrm{~Hz}, \mathrm{C}_{\text {quat }}\right) .{ }^{19} \mathrm{~F} \mathrm{NMR}\left(\mathrm{CDCl}_{3}\right): \delta-219.6(\mathrm{dt}, \mathrm{J}=12.6 \mathrm{~Hz}, 46.9 \mathrm{~Hz}$,

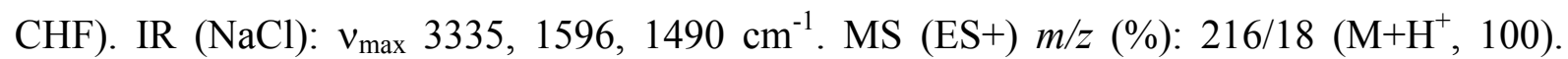
HRMS calcd for $\mathrm{C}_{11} \mathrm{H}_{16} \mathrm{~N}^{35} \mathrm{ClF}\left(\mathrm{M}+\mathrm{H}^{+}\right)$216.09498, found 216.09481.

$\boldsymbol{N}$-Butyl- $\boldsymbol{N}$-(2-fluoro-1-phenylethyl)amine 5c. Flash chromatography (hexane/EtOAc/Et ${ }_{3} \mathrm{~N}$ 95:4.9:0.1, $\left.\mathrm{R}_{\mathrm{f}}=0.18\right)$, yield 76\%. ${ }^{1} \mathrm{H} \mathrm{NMR}\left(\mathrm{CDCl}_{3}\right): \delta 0.87(3 \mathrm{H}, \mathrm{t}, \mathrm{J}=$ 7.1Hz, Me), 1.26-1.38 (2H, m, $\left.\mathrm{CH}_{2}\right), 1.46\left(2 \mathrm{H}\right.$, quint, $\left.\mathrm{J}=7.1 \mathrm{~Hz}, \mathrm{CH}_{2}\right), 1.70(1 \mathrm{H}, \mathrm{s}(\mathrm{b}), \mathrm{NH})$, $2.49\left(2 \mathrm{H}, \mathrm{t}, \mathrm{J}=7.0 \mathrm{~Hz}, \mathrm{NCH}_{2}\right), 4.01\left(1 \mathrm{H}, \mathrm{ddd}, \mathrm{J}_{\mathrm{HF}}=13.0 \mathrm{~Hz}, \mathrm{~J}_{\mathrm{HH}}=8.8 \mathrm{~Hz}, \mathrm{~J}_{\mathrm{HH}}=4.2 \mathrm{~Hz}, \mathrm{NCH}\right)$, $4.37\left(1 \mathrm{H}, \mathrm{dt}, \mathrm{J}_{\mathrm{HF}}=47.4 \mathrm{~Hz}, \mathrm{~J}_{\mathrm{HH}}=8.8 \mathrm{~Hz}, \mathrm{C}(\mathrm{H}) H \mathrm{~F}\right), 4.45\left(1 \mathrm{H}, \mathrm{ddd}, \mathrm{J}_{\mathrm{HF}}=47.4 \mathrm{~Hz}, \mathrm{~J}_{\mathrm{HH}}=8.8 \mathrm{~Hz}\right.$, $\left.\mathrm{J}_{\mathrm{HH}}=4.2 \mathrm{~Hz}, \mathrm{C}(H) \mathrm{HF}\right), 7.26-7.36\left(5 \mathrm{H}, \mathrm{m}, 5 \mathrm{x} \mathrm{CH} \mathrm{Cr}_{\mathrm{ar}}\right){ }^{13} \mathrm{C} \mathrm{NMR}\left(\mathrm{CDCl}_{3}\right): \delta 14.1\left(\mathrm{CH}_{3}\right), 20.5$ $\left(\mathrm{CH}_{2}\right), 32.4\left(\mathrm{CH}_{2}\right), 47.3\left(\mathrm{NCH}_{2}\right), 63.2(\mathrm{~d}, \mathrm{~J}=18.5 \mathrm{~Hz}, \mathrm{NCH}), 87.2\left(\mathrm{~d}, \mathrm{~J}=174.1 \mathrm{~Hz}, \mathrm{CH}_{2} \mathrm{~F}\right)$, 
$127.8\left(2 \times \mathrm{CH}_{\mathrm{ar}}\right), 128.0\left(\mathrm{CH}_{\mathrm{ar}}\right), 128.7\left(2 \times \mathrm{CH}_{\mathrm{ar}}\right), 138.9\left(\mathrm{~d}, \mathrm{~J}=8.1 \mathrm{~Hz}, \mathrm{C}_{\text {quat }}\right) .{ }^{19} \mathrm{~F} \mathrm{NMR}$ $\left(\mathrm{CDCl}_{3}\right): \delta-218.4(\mathrm{dt}, \mathrm{J}=13.0 \mathrm{~Hz}, 47.4 \mathrm{~Hz}, \mathrm{CHF}) . \mathrm{IR}(\mathrm{NaCl}): v_{\max } 3339,1454 \mathrm{~cm}^{-1} . \mathrm{MS}(\mathrm{ES}+)$ $m / z(\%): 196\left(\mathrm{M}+\mathrm{H}^{+}, 100\right)$. HRMS calcd for $\mathrm{C}_{12} \mathrm{H}_{19} \mathrm{NF}\left(\mathrm{M}+\mathrm{H}^{+}\right)$196.14960, found 196.14986.

\section{Synthesis of $\alpha$-fluoroketones 8}

2-Fluorocyclohexanone $8 \mathbf{a}^{6 \mathrm{~m}}$ For the synthetic procedure is referred to the synthesis of fluorinated imines 3 and the hydrolysis to fluorinated ketones 4 (see above). Flash chromatography (hexane/EtOAc 9:1, $\mathrm{R}_{\mathrm{f}}=0.13$ ), yield 86\%. Spectral data were in accordance with literature data. ${ }^{6 \mathrm{~m}}$ Copies of ${ }^{1} \mathrm{H}$ NMR and ${ }^{13} \mathrm{C}$ NMR spectra as an indication of purity are included in chapter III of the supporting information file.

2-Fluoro-5-nonanone 8b. ${ }^{17}$ Flash chromatography (hexane/EtOAc 94:6, $\mathrm{R}_{\mathrm{f}}=0.52$ ), yield $65 \%$. Spectral data were in accordance with literature data. ${ }^{17}$ Copies of ${ }^{1} \mathrm{H}$ NMR and ${ }^{13} \mathrm{C}$ NMR spectra as an indication of purity are included in chapter III of the supporting information file.

1-Fluoro-1-phenylacetone 8c. ${ }^{16}$ Flash chromatography (hexane/EtOAc 9:1, $\mathrm{R}_{\mathrm{f}}=$ 0.32 ), yield $61 \%$. Spectral data were in accordance with literature data. ${ }^{16}$ Copies of ${ }^{1} \mathrm{H}$ NMR and ${ }^{13} \mathrm{C}$ NMR spectra as an indication of purity are included in chapter III of the supporting information file.

\section{Synthesis of $\alpha, \alpha$-difluoroimines 9}

$N$-(2,2-Difluoro-1-phenylethylidene)isopropylamine 9a. In a flame dried $100 \mathrm{ml}$ flask, a heterogeneous mixture of $5.87 \mathrm{~g}$ (18.63 mmol, 3 equiv.) of NFSI ( $N$ fluorobenzenesulfonimide) and $1.72 \mathrm{~g}$ (12.42 mmol, 2 equiv.) of $\mathrm{K}_{2} \mathrm{CO}_{3}$ and $8 \mathrm{~g}$ of $4 \AA$ molecular sieves (dried at $120^{\circ} \mathrm{C}$ ) in $50 \mathrm{ml}$ of acetonitrile and $10 \mathrm{ml}$ of DMF was vigorously stirred under $\mathrm{N}_{2}$ atmosphere for 15 minutes at room temperature. Subsequently, $1.00 \mathrm{~g}$ (6.21 mmol) of freshly distilled $N$-(1-phenylethylidene)isopropylamine $\mathbf{2 a}$ was added dropwise via a syringe. The mixture was stirred for 15 hours. Subsequently, an excess of triethylamine (5 $\mathrm{ml}$ ) was added at $0^{\circ} \mathrm{C}$ and stirring was continued for 2 minutes. The reaction mixture was filtered over Celite and the solids were washed 3 times with $20 \mathrm{ml}$ of diethyl ether. Subsequently, the filtrate was poured in $100 \mathrm{ml}$ of aqueous $0.5 \mathrm{M} \mathrm{NaOH}$. After separation of the organic layer, the aqueous phase was extracted with $3 \times 25 \mathrm{ml}$ of diethyl ether. To remove the DMF, the combined organic phases were washed with a saturated aqueous $\mathrm{NaCl}$-solution $(2 \times 100 \mathrm{ml})$ and subsequently dried with $\mathrm{MgSO}_{4} / \mathrm{K}_{2} \mathrm{CO}_{3}$ (10:1). Filtration of the drying agents and evaporation of the solvents in vacuo yielded difluoroimine 9a (together with 18\% 
of 2-fluoroacetophenone). Other imines 9b-9e were obtained using an analogous procedure and were obtained without significant amounts of the corresponding monofluoroimines or ketones. Purification of imines $9 \mathbf{9 b - 9 e}$ could be established by column chromatography (short column) using dry solvents and silica that was dried at high vacuum $(0.01 \mathrm{mmHg})$ at $100^{\circ} \mathrm{C}$ for $5 \mathrm{~h}$ and treated with $\mathrm{Et}_{3} \mathrm{~N}$ directly after cooling to room temperature. Yield of $9 \mathrm{a} 74 \%$. Mp $41^{\circ} \mathrm{C} .{ }^{1} \mathrm{H}$ NMR $\left(\mathrm{CDCl}_{3}\right): \delta 1.12(6 \mathrm{H}, \mathrm{d}, \mathrm{J}=6.2 \mathrm{~Hz}, 2 \mathrm{xMe}), 3.59\left(1 \mathrm{H}, \mathrm{sept}, \mathrm{J}=6.2 \mathrm{~Hz}, \mathrm{CHMe}_{2}\right)$, $6.13\left(1 \mathrm{H}, \mathrm{t}, \mathrm{J}=55.4 \mathrm{~Hz}, \mathrm{CHF}_{2}\right), 7.20-7.25\left(2 \mathrm{H}, \mathrm{m}, 2 \mathrm{xCH}_{\mathrm{ar}}\right), 7.41-7.48\left(3 \mathrm{H}, \mathrm{m}, 3 \mathrm{xCH}_{\mathrm{ar}}\right) .{ }^{13} \mathrm{C}$ NMR $\left(\mathrm{CDCl}_{3}\right): \delta 2 \times 23.2\left(2 \times \mathrm{CH}_{3}\right), 52.3(\mathrm{CH}), 115.4\left(\mathrm{t}, \mathrm{J}=244.0 \mathrm{~Hz}, \mathrm{CHF}_{2}\right), 127.6(2 \mathrm{x}$ $\left.\mathrm{CH}_{\mathrm{ar}}\right), 128.4\left(2 \times \mathrm{CH}_{\mathrm{ar}}\right), 129.2\left(\mathrm{CH}_{\mathrm{ar}}\right), 131.2\left(\mathrm{C}_{\text {quat }}\right), 161.1(\mathrm{t}, \mathrm{J}=27.7 \mathrm{~Hz}, \mathrm{C}=\mathrm{N}) .{ }^{19} \mathrm{~F}$ NMR $\left(\mathrm{CDCl}_{3}\right): \delta-117.8(2 \mathrm{~F}, \mathrm{~d}, \mathrm{~J}=55.4 \mathrm{~Hz})$. IR (KBr) $v_{\max } 1660 \mathrm{~cm}^{-1}$. MS (ES+) $\mathrm{m} / z(\%): 198$ $\left(\mathrm{M}+\mathrm{H}^{+}, 100\right)$. Copies of ${ }^{1} \mathrm{H}$ NMR and ${ }^{13} \mathrm{C}$ NMR spectra are included in chapter III of the supporting information file.

$N$-(2,2-Difluoro-1-phenylpropylidene)isopropylamine 9b. Flash chromatography (hexane/EtOAc/Et ${ }_{3} \mathrm{~N}$ 95:4.8:0.2, $\left.\mathrm{R}_{\mathrm{f}}=0.6\right)$, yield 54\%. ${ }^{1} \mathrm{H}$ NMR $\left(\mathrm{CDCl}_{3}\right): \delta 1.08(6 \mathrm{H}, \mathrm{d}$, $\left.\mathrm{J}=6.1 \mathrm{~Hz}, \mathrm{CH}(\underline{\mathrm{Me}})_{2}\right), 1.86\left(3 \mathrm{H}, \mathrm{t}, \mathrm{J}=17.9 \mathrm{~Hz}, \mathrm{CH}_{3}\right), 3.44\left(1 \mathrm{H}\right.$, sept, J=6.1Hz, $\left.\mathrm{C} \underline{\mathrm{H}}(\mathrm{Me})_{2}\right), 7.15-$ $7.18\left(2 \mathrm{H}, \mathrm{m}, 2 \mathrm{xCH}_{\mathrm{ar}}\right), 7.39-7.46\left(3 \mathrm{H}, \mathrm{m}, 3 \mathrm{xCH}_{\mathrm{ar}}\right) .{ }^{13} \mathrm{C} \mathrm{NMR}\left(\mathrm{CDCl}_{3}\right): \delta 21.6(\mathrm{t}, \mathrm{J}=26.5 \mathrm{~Hz}$, $\left.\mathrm{CH}_{3}\right), 23.4\left(\mathrm{CH}(\underline{\mathrm{Me}})_{2}\right), 52.6\left(\underline{\mathrm{CH}}(\mathrm{Me})_{2}\right), 120.9\left(\mathrm{t}, \mathrm{J}=239.4 \mathrm{~Hz}, \mathrm{CF}_{2}\right), 127.7\left(2 \mathrm{xCH}_{\mathrm{ar}}\right), 128.4$ $\left(2 \mathrm{xCH}_{\mathrm{ar}}\right), 128.9\left(\mathrm{CH}_{\mathrm{ar}}\right), 133.1\left(\mathrm{C}_{\mathrm{ar}, \text { quat }}\right), 162.5(\mathrm{t}, \mathrm{J}=31.2 \mathrm{~Hz}, \mathrm{C}=\mathrm{N}) .{ }^{19} \mathrm{~F} \mathrm{NMR}\left(\mathrm{CDCl}_{3}\right): \delta-92.4$ (2F, q, J=17.9Hz). IR ( $\mathrm{NaCl}): v_{\max } 1657 \mathrm{~cm}^{-1}$. MS (ES+): m/z(\%): $212\left(\mathrm{M}+\mathrm{H}^{+}, 100\right)$. Copies of ${ }^{1} \mathrm{H}$ NMR and ${ }^{13} \mathrm{C}$ NMR spectra are included in chapter III of the supporting information file.

N-(2,2-Difluoro-1-phenylbutylidene)isopropylamine 9c. Flash chromatography (hexane/EtOAc/Et ${ }_{3} \mathrm{~N}$ 94:4:2, $\left.\mathrm{R}_{\mathrm{f}}=0.3\right)$, yield 63\%. ${ }^{1} \mathrm{H} \mathrm{NMR}\left(\mathrm{CDCl}_{3}\right): \delta 1.07(3 \mathrm{H}, \mathrm{t}, \mathrm{J}=7.5 \mathrm{~Hz}$, $\left.\mathrm{CH}_{2} \underline{\mathrm{Me}}\right), 1.09\left(6 \mathrm{H}, \mathrm{d}, \mathrm{J}=6.1 \mathrm{~Hz}, \mathrm{CH}(\underline{\mathrm{Me}})_{2}\right), 2.18\left(2 \mathrm{H}, \mathrm{tq}, \mathrm{J}_{\mathrm{HF}}=16.7 \mathrm{~Hz}, \mathrm{~J}_{\mathrm{HH}}=7.5 \mathrm{~Hz}, \mathrm{C}_{2} \mathrm{Me}\right)$, $3.49\left(1 \mathrm{H}\right.$, sept, J=6.1Hz, $\left.\mathrm{C} \underline{\mathrm{H}}(\mathrm{Me})_{2}\right), 7.14-7.17\left(2 \mathrm{H}, \mathrm{m}, 2 \mathrm{xCH}_{\mathrm{ar}}\right), 7.38-7.44\left(3 \mathrm{H}, \mathrm{m}, 3 \mathrm{xCH}_{\mathrm{ar}}\right)$. ${ }^{13} \mathrm{C} \mathrm{NMR}\left(\mathrm{CDCl}_{3}\right.$, int. ref.=77.13ppm): $\delta 6.5\left(\mathrm{t}, \mathrm{J}=5.2 \mathrm{~Hz}, \mathrm{CH}_{2} \underline{\mathrm{Me}}\right), 23.5\left(\mathrm{CH}(\underline{\mathrm{Me}})_{2}\right), 27.8(\mathrm{t}$, $\left.\mathrm{J}=25.4 \mathrm{~Hz}, \underline{\mathrm{CH}}_{2} \mathrm{CF}_{2}\right), 52.7\left(\underline{\mathrm{CH}}(\mathrm{Me})_{2}\right), 121.3\left(\mathrm{t}, \mathrm{J}=242.9 \mathrm{~Hz}, \mathrm{CF}_{2}\right), 127.6\left(2 \mathrm{xCH}_{\mathrm{ar}}\right), 128.4$ $\left(2 \mathrm{xCH}_{\mathrm{ar}}\right), 128.9\left(\mathrm{CH}_{\mathrm{ar}}\right), 133.3\left(\mathrm{C}_{\mathrm{ar} \text {,quat }}\right), 162.2(\mathrm{t}, \mathrm{J}=30.0 \mathrm{~Hz}, \mathrm{C}=\mathrm{N}) \cdot{ }^{19} \mathrm{~F} \mathrm{NMR}\left(\mathrm{CDCl}_{3}\right): \delta-101.6$ (2F, s(br)). IR (NaCl): $v_{\max } 1663 \mathrm{~cm}^{-1}$. MS (ES+): m/z(\%): $226\left(\mathrm{M}+\mathrm{H}^{+}, 100\right)$. Copies of ${ }^{1} \mathrm{H}$ NMR and ${ }^{13} \mathrm{C}$ NMR spectra are included in chapter III of the supporting information file.

$N$-(2,2-Difluoro-1-phenylbutylidene)cyclohexylamine 9d. Flash chromatography (hexane/EtOAc/Et $3 \mathrm{~N}$ 95:4.8:0.2, $\left.\mathrm{R}_{\mathrm{f}}=0.5\right)$, yield 63\%. ${ }^{1} \mathrm{H} \mathrm{NMR}\left(\mathrm{CDCl}_{3}\right): \delta$ 0.82-1.29 $(4 \mathrm{H}, \mathrm{m}$, $\left.4 \times \mathrm{CH}_{\text {cyclohexyl }}\right), 1.06\left(3 \mathrm{H}, \mathrm{t}, \mathrm{J}=7.4 \mathrm{~Hz}, \mathrm{CH}_{3}\right), 1.49-1.59$ (4H, m, 4 x $\left.\mathrm{CH}_{\text {cyclohexyl }}\right), 1.67-1.73$ $\left(2 \mathrm{H}, \mathrm{m}, 2 \times \mathrm{CH}_{\text {cyclohexyl }}\right), 2.17\left(2 \mathrm{H}, \mathrm{tq}, \mathrm{J}_{\mathrm{HH}}=7.4 \mathrm{~Hz}, \mathrm{~J}_{\mathrm{HF}}=16.7 \mathrm{~Hz}, \mathrm{CH}_{2}\right), 3.12$ (1H, quint, 
$\mathrm{J}=7.1 \mathrm{~Hz}, \mathrm{NCH}), 7.13-7.18\left(2 \mathrm{H}, \mathrm{m}, 2 \mathrm{xCH}_{\mathrm{ar}}\right), 7.38-7.43\left(3 \mathrm{H}, \mathrm{m}, 3 \mathrm{xCH}_{\mathrm{ar}}\right) .{ }^{13} \mathrm{C} \mathrm{NMR}\left(\mathrm{CDCl}_{3}\right.$, int. ref.=77.00ppm): $\delta 6.4\left(\mathrm{t}, \mathrm{J}=4.6 \mathrm{~Hz}, \mathrm{CH}_{2} \underline{\mathrm{Me}}\right), 24.0\left(2 \times \mathrm{CH}_{2, \mathrm{cHex}}\right), 25.5\left(\mathrm{CH}_{2, \mathrm{cHex}}\right), 27.7$ (t, $\left.\mathrm{J}=25.4 \mathrm{~Hz}, \underline{\mathrm{CH}}_{2} \mathrm{CF}_{2}\right), 32.3(\mathrm{NCH}), 121.2\left(\mathrm{t}, \mathrm{J}=242.3 \mathrm{~Hz}, \mathrm{CF}_{2}\right), 127.5\left(2 \mathrm{xCH}_{\mathrm{ar}}\right), 128.3\left(2 \mathrm{xCH}_{\mathrm{ar}}\right)$, $128.7\left(\mathrm{CH}_{\mathrm{ar}}\right), 133.2\left(\mathrm{C}_{\mathrm{ar} \text {,quat }}\right), 162.1(\mathrm{t}, \mathrm{J}=30.6 \mathrm{~Hz}, \mathrm{C}=\mathrm{N}) .{ }^{19} \mathrm{~F}$ NMR $\left(\mathrm{CDCl}_{3}\right): \delta-101.3(2 \mathrm{~F}$, s(br)). IR (NaCl): $v_{\max } 1660 \mathrm{~cm}^{-1}$. MS (ES+): m/z(\%): $266\left(\mathrm{M}+\mathrm{H}^{+}, 100\right)$. Copies of ${ }^{1} \mathrm{H}$ NMR and ${ }^{13} \mathrm{C}$ NMR spectra are included in chapter III of the supporting information file.

$\boldsymbol{N}$-(2,2-Difluoro-1-phenylpentylidene)isopropylamine 9e. $\mathrm{Bp} 80^{\circ} \mathrm{C}(1 \mathrm{mmHg})$, yield: $49 \%$. ${ }^{1} \mathrm{H}$ NMR $\left(\mathrm{CDCl}_{3}\right): \delta 0.98(3 \mathrm{H}, \mathrm{t}, \mathrm{J}=7.8 \mathrm{~Hz}, \mathrm{Me}), 1.09(6 \mathrm{H}, \mathrm{d}, \mathrm{J}=6.3 \mathrm{~Hz}, 2 \mathrm{xMe})$, $1.58\left(2 \mathrm{H}\right.$, sext, $\left.\mathrm{J}=7.8 \mathrm{~Hz}, \mathrm{CH}_{2} \mathrm{Me}\right), 2.13\left(2 \mathrm{H}, \mathrm{tt}, \mathrm{J}_{\mathrm{HF}}=17,0 \mathrm{~Hz}, \mathrm{~J}_{\mathrm{HH}}=7,8 \mathrm{~Hz}, \mathrm{CH}_{2} \mathrm{CF}_{2}\right), 3.44(1 \mathrm{H}$, sept, J=6.3Hz, $\left.\underline{\mathrm{HMe}}_{2}\right), 7.12-7.18\left(2 \mathrm{H}, \mathrm{m}, 2 \mathrm{xCH}_{\mathrm{ar}}\right), 7.38-7.43\left(3 \mathrm{H}, \mathrm{m}, 3 \mathrm{xCH}_{\mathrm{ar}}\right) .{ }^{13} \mathrm{C} \mathrm{NMR}$



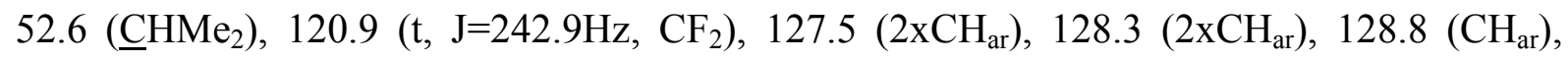
$133.2\left(\mathrm{C}_{\text {ar,quat }}\right), 162.3(\mathrm{t}, \mathrm{J}=30.6 \mathrm{~Hz}, \mathrm{C}=\mathrm{N}) .{ }^{19} \mathrm{~F}$ NMR $\left(\mathrm{CDCl}_{3}\right): \delta-99.8(2 \mathrm{~F}, \mathrm{t}, \mathrm{J}=17,0 \mathrm{~Hz}) . \mathrm{IR}$ $(\mathrm{NaCl}): v_{\max } 1657 \mathrm{~cm}^{-1}$. MS (ES+): m/z(\%): $240\left(\mathrm{M}+\mathrm{H}^{+}, 100\right)$. Copies of ${ }^{1} \mathrm{H} \mathrm{NMR}$ and ${ }^{13} \mathrm{C}$ NMR spectra are included in chapter III of the supporting information file.

\section{Synthesis of $\beta, \beta$-difluoroamines 10}

$N$-(2,2-Difluoro-1-phenylethyl)- $N$-isopropylamine 10a. For the synthetic procedures of the reductions of $\alpha, \alpha$-difluoroimines $\mathbf{9}$ towards amines $\mathbf{1 0}$ is referred to the synthesis of amines 5 from $\alpha$-fluoroimines 3 . Amine 10a was purified by flash chromatography using a short plug of silicagel (hexane/EtOAc 95:5, $\mathrm{R}_{\mathrm{f}}=0.42$ ), yield 82\%. ${ }^{1} \mathrm{H} \mathrm{NMR}\left(\mathrm{CDCl}_{3}\right): \delta 1.03$ $(3 \mathrm{H}, \mathrm{d}, \mathrm{J}=6.3 \mathrm{~Hz}, \mathrm{Me}), 1.04(3 \mathrm{H}, \mathrm{d}, \mathrm{J}=6.3 \mathrm{~Hz}, \mathrm{Me}), 1.45$ (1H, s(br), NH), 2.69 (1H, sept, $\left.\mathrm{J}=6.3 \mathrm{~Hz}, \mathrm{C} \underline{\mathrm{H}}(\mathrm{Me})_{2}\right), 4.03\left(1 \mathrm{H}, \mathrm{ddd}, \mathrm{J}_{\mathrm{HF}}=13.3 \mathrm{~Hz}, \mathrm{~J}_{\mathrm{HF}}=10.6 \mathrm{~Hz}, \mathrm{~J}_{\mathrm{HH}}=4.4 \mathrm{~Hz}, \mathrm{CHCHF}_{2}\right), 5.79$ $\left(1 \mathrm{H}, \mathrm{dt}, \mathrm{J}_{\mathrm{HF}}=56.4 \mathrm{~Hz}, \mathrm{~J}_{\mathrm{HH}}=4.4 \mathrm{~Hz}, \mathrm{CHF}_{2}\right), 7.25-7.40\left(5 \mathrm{H}, \mathrm{m}, 5 \mathrm{xCH}_{\mathrm{ar}}\right) .{ }^{13} \mathrm{C} \mathrm{NMR}\left(\mathrm{CDCl}_{3}\right.$, int. ref.=77.13ppm): $\delta 21.9(\mathrm{Me}), 24.1(\mathrm{Me}), 45.5\left(\underline{\mathrm{CH}}(\mathrm{Me})_{2}\right), 62.3\left(\mathrm{t}, \mathrm{J}=87.0 \mathrm{~Hz}, \underline{\mathrm{CCF}_{2}}\right), 117.2(\mathrm{t}$, $\left.\mathrm{J}=245.2 \mathrm{~Hz}, \mathrm{CF}_{2}\right), 128.5\left(\mathrm{CH}_{\mathrm{ar}}\right), 128.5\left(2 \mathrm{xCH}_{\mathrm{ar}}\right), 128.7\left(2 \mathrm{xCH}_{\mathrm{ar}}\right), 136.5\left(\mathrm{C}_{\mathrm{ar}, \text { quat }}\right){ }^{19} \mathrm{~F} \mathrm{NMR}$ $\left(\mathrm{CDCl}_{3}\right): \delta-123.7\left(1 \mathrm{~F}, \mathrm{ddd}, \mathrm{J}_{\mathrm{FF}}=277.0 \mathrm{~Hz}, \mathrm{~J}_{\mathrm{HF}}=56.7 \mathrm{~Hz}, \mathrm{~J}_{\mathrm{HF}}=10.6 \mathrm{~Hz}\right) ;-125.44 \quad(1 \mathrm{~F}$, ddd, $\left.\mathrm{J}_{\mathrm{FF}}=277.0 \mathrm{~Hz}, \mathrm{~J}_{\mathrm{HF}}=56.4 \mathrm{~Hz}, \mathrm{~J}_{\mathrm{HF}}=13.3 \mathrm{~Hz}\right) . \mathrm{IR}(\mathrm{NaCl}): v_{\max } 3339 \mathrm{~cm}^{-1} . \mathbf{M S}(\mathrm{ES}+): \mathrm{m} / \mathrm{z}(\%): 200$ $\left(\mathrm{M}+\mathrm{H}^{+}, 100\right)$. Copies of ${ }^{1} \mathrm{H}$ NMR and ${ }^{13} \mathrm{C}$ NMR spectra are included in chapter III of the supporting information file.

$N$-(2,2-Difluoro-1-phenylpropyl)- $N$-isopropylamine 10b. Purification by acid-base extraction $\left(\mathrm{Et}_{2} \mathrm{O} / \mathrm{HCl} ; \mathrm{NaOH}\right)$, yield 90\%. ${ }^{1} \mathrm{H} \mathrm{NMR}\left(\mathrm{CDCl}_{3}\right): \delta 1.01(3 \mathrm{H}, \mathrm{d}, \mathrm{J}=6.3 \mathrm{~Hz}, \mathrm{Me})$, $1.02(3 \mathrm{H}, \mathrm{d}, \mathrm{J}=6.3 \mathrm{~Hz}, \mathrm{Me}), 1.53\left(3 \mathrm{H}, \mathrm{t}, \mathrm{J}=19.0 \mathrm{~Hz}, \mathrm{CH}_{3} \mathrm{CF}_{2}\right), 2.62(1 \mathrm{H}$, sept, $\mathrm{J}=6.3 \mathrm{~Hz}$, 
$\mathrm{NCH}), 3.99(1 \mathrm{H}, \mathrm{t}, \mathrm{J}=11.6 \mathrm{~Hz}, \mathrm{NCH}), 7.26-7.38\left(5 \mathrm{H}, \mathrm{m}, 5 \mathrm{x} \mathrm{CH} \mathrm{CH}_{\mathrm{ar}}\right) .{ }^{13} \mathrm{C} \mathrm{NMR}\left(\mathrm{CDCl}_{3}\right.$, int. ref.=77.00ppm): $\delta 20.8\left(\mathrm{t}, \mathrm{J}=27.1 \mathrm{~Hz}, \mathrm{CH}_{3}\right), 21.5\left(\mathrm{CH}_{3}\right), 24.1\left(\mathrm{CH}_{3}\right), 45.4(\mathrm{NCH}), 64.9(\mathrm{t}, \mathrm{J}=$ $\left.25.4 \mathrm{~Hz}, \underline{\mathrm{C}} \mathrm{HCF}_{2}\right), 124.1\left(\mathrm{t}, \mathrm{J}=242.9 \mathrm{~Hz}, \mathrm{CF}_{2}\right), 128.0\left(\mathrm{CH}_{\mathrm{ar}}\right), 128.2\left(2 \mathrm{x} \mathrm{CH}_{\mathrm{ar}}\right), 128.6(2 \mathrm{x}$ $\left.\mathrm{CH}_{\mathrm{ar}}\right), 137.6\left(\mathrm{C}_{\text {quat }}\right) .{ }^{19} \mathrm{~F}$ NMR $\left(\mathrm{CDCl}_{3}\right): \delta-98.0\left(\mathrm{~s}(\mathrm{br}), \mathrm{CF}_{2}\right) . \mathrm{IR}(\mathrm{NaCl}): v_{\max } 3337 \mathrm{~cm}^{-1} . \mathrm{MS}$ $(\mathrm{ES}+) \mathrm{m} / z(\%): 214\left(\mathrm{M}+\mathrm{H}^{+}, 100\right)$. Copies of ${ }^{1} \mathrm{H}$ NMR and ${ }^{13} \mathrm{C}$ NMR spectra are included in chapter III of the supporting information file.

$\mathrm{N}$-(2,2-Difluoro-1-phenylbutyl)- $\mathrm{N}$-isopropylamine 10c. Flash chromatography (hexane/EtOAc/ $\mathrm{Et}_{3} \mathrm{~N}$ 94:4:2, $\left.\mathrm{R}_{\mathrm{f}}=0.33\right)$, yield 60\%. ${ }^{1} \mathrm{H} \mathrm{NMR}\left(\mathrm{CDCl}_{3}\right): \delta$ 0.96-1.01 $(9 \mathrm{H}, \mathrm{m}$, $3 x \mathrm{Me}), 1.52(1 \mathrm{H}, \mathrm{s}(\mathrm{br}), \mathrm{NH}), 1.68-2.04\left(2 \mathrm{H}, \mathrm{m}, \mathrm{C}_{2} \mathrm{Me}\right), 2.60\left(1 \mathrm{H}, \mathrm{sept}, \mathrm{J}=6.3 \mathrm{~Hz}, \mathrm{C} \underline{\mathrm{H}}(\mathrm{Me})_{2}\right)$, $3.99\left(1 \mathrm{H}, \mathrm{d}, \mathrm{J}_{\mathrm{HF}}=11.5 \mathrm{~Hz}, \mathrm{CHCF}_{2}\right), 7.24-7.41\left(5 \mathrm{H}, \mathrm{m}, 5 \mathrm{xCH}_{\mathrm{ar}}\right) .{ }^{13} \mathrm{C} \mathrm{NMR}\left(\mathrm{CDCl}_{3}\right.$, int. ref.=77.13ppm): $\delta 6.0\left(\mathrm{CH}_{2} \underline{\mathrm{Me}}\right), 21.6(\mathrm{CH}(\underline{\mathrm{Me}}) \mathrm{Me}), 24.3(\mathrm{CH}(\mathrm{Me}) \underline{\mathrm{Me}}), 27.3(\mathrm{t}, \mathrm{J}=25.4 \mathrm{~Hz}$, $\left.\underline{\mathrm{CH}}_{2} \mathrm{CF}_{2}\right), 45.4\left(\underline{\mathrm{CH}}(\mathrm{Me})_{2}\right), 63.8(\mathrm{t}, \mathrm{J}=25.4 \mathrm{~Hz}, \underline{\mathrm{CHCF}})_{2}, 124.9\left(\mathrm{t}, \mathrm{J}=245.8 \mathrm{~Hz}, \mathrm{CF}_{2}\right), 128.0$ $\left(\mathrm{CH}_{\mathrm{ar}}\right), 128.4\left(2 \mathrm{xCH}_{\mathrm{ar}}\right), 128.8\left(2 \mathrm{xCH}_{\mathrm{ar}}\right), 137.7\left(\mathrm{C}_{\mathrm{ar} \text {,quat }}\right) .{ }^{19} \mathrm{~F}$ NMR $\left(\mathrm{CDCl}_{3}\right): \delta-109.06(2 \mathrm{~F}$, $\left.\mathrm{s}(\mathrm{br}), \mathrm{CF}_{2}\right)$. IR $(\mathrm{NaCl}): v_{\max } 3340 \mathrm{~cm}^{-1} . \mathrm{MS}(\mathrm{ES}+): \mathrm{m} / \mathrm{z}(\%): 228\left(\mathrm{M}+\mathrm{H}^{+}, 100\right)$. Copies of ${ }^{1} \mathrm{H}$ $\mathrm{NMR}$ and ${ }^{13} \mathrm{C}$ NMR spectra are included in chapter III of the supporting information file.

$\mathrm{N}$-Cyclohexyl- $\mathrm{N}$-(2,2-difluoro-1-phenylbutyl)amine 10d. Purification by acid-base extraction $\left(\mathrm{Et}_{2} \mathrm{O} / \mathrm{HCl} ; \mathrm{NaOH}\right)$, yield 94\%. ${ }^{1} \mathrm{H} \mathrm{NMR}\left(\mathrm{CDCl}_{3}\right): \delta 0.98(3 \mathrm{H}, \mathrm{t}, \mathrm{J}=7.6 \mathrm{~Hz}$, $\mathrm{CH}_{2} \underline{\mathrm{C}}_{3}$ ), 1.01-1.23 (4H, m, 4 x CH $\left.\mathrm{CH}_{\text {cyclohexyl }}\right), 1.43-2.04\left(8 \mathrm{H}, \mathrm{m}, 6 \times \mathrm{CH}_{\text {cyclohexyl }}\right.$ and $\mathrm{CH}_{2} \mathrm{CF}_{2}$ ), 2.16-2.30 (1H, m, NCH), $4.05\left(1 \mathrm{H}, \mathrm{t}, \mathrm{J}=12.4 \mathrm{~Hz}, \mathrm{CHCF}_{2}\right), 7.28-7.36\left(5 \mathrm{H}, \mathrm{m}, 5 \mathrm{x} \mathrm{CH}_{\mathrm{ar}}\right){ }^{13} \mathrm{C}$ NMR $\left(\mathrm{CDCl}_{3}\right.$, int. ref.=77.00ppm): $\delta 5.8\left(\mathrm{t}, \mathrm{J}=5.2 \mathrm{~Hz}, \mathrm{CH}_{3}\right), 24.4\left(\mathrm{CH}_{2}\right), 24.9\left(\mathrm{CH}_{2}\right), 26.0$ $\left(\mathrm{CH}_{2}\right), 27.0\left(\mathrm{t}, \mathrm{J}=25.4 \mathrm{~Hz}, \underline{\mathrm{CH}}_{2} \mathrm{CF}_{2}\right), 32.3\left(\mathrm{CH}_{2}\right), 34.6\left(\mathrm{CH}_{2}\right), 53.0(\mathrm{NCH}), 63.0(\mathrm{t}, \mathrm{J}=24.8 \mathrm{~Hz}$, $\left.\underline{\mathrm{C}} \mathrm{HCF}_{2}\right), 124.8\left(\mathrm{t}, \mathrm{J}=245.8 \mathrm{~Hz}, \mathrm{CF}_{2}\right), 127.8\left(\mathrm{CH}_{\mathrm{ar}}\right), 128.2\left(2 \times \mathrm{CH}_{\mathrm{ar}}\right), 128.7\left(2 \times \mathrm{CH}_{\mathrm{ar}}\right), 137.8$ $\left(\mathrm{C}_{\text {ar,quat }}\right){ }^{19} \mathrm{~F}$ NMR $\left(\mathrm{CDCl}_{3}\right): \delta-109.10(2 \mathrm{~F}, \mathrm{~s}(\mathrm{br}))$. IR $(\mathrm{NaCl}): v_{\max } 3338 \mathrm{~cm}^{-1}$. MS (ES+): $\mathrm{m} / \mathrm{z}(\%): 268\left(\mathrm{M}+\mathrm{H}^{+}, 100\right)$. Copies of ${ }^{1} \mathrm{H} \mathrm{NMR}$ and ${ }^{13} \mathrm{C}$ NMR spectra are included in chapter III of the supporting information file.

$\boldsymbol{N}$-(2,2-Difluoro-1-phenylpentyl)- $\boldsymbol{N}$-isopropylamine 10e. Yield $81 \%$. ${ }^{1} \mathrm{H} \quad \mathrm{NMR}$ $\left(\mathrm{CDCl}_{3}\right): \delta 0.90(3 \mathrm{H}, \mathrm{t}, \mathrm{J}=7.4 \mathrm{~Hz}, \mathrm{Me}), 0.99(3 \mathrm{H}, \mathrm{d}, \mathrm{J}=6.2 \mathrm{~Hz}, \mathrm{Me}), 1.00(3 \mathrm{H}, \mathrm{d}, \mathrm{J}=6.2 \mathrm{~Hz}, \mathrm{Me})$, 1.41-1.60 (2H, m, $\left.\underline{\mathrm{C}}_{2} \mathrm{Me}\right), 1.63-1.97\left(2 \mathrm{H}, \mathrm{m}, \mathrm{CH}_{2} \mathrm{CF}_{2}\right), 2.59\left(1 \mathrm{H}\right.$, sept, J=6.2Hz, $\left.\underline{\mathrm{HMe}}_{2}\right)$, $3.97\left(1 \mathrm{H}, \mathrm{t}, \mathrm{J}=12.4 \mathrm{~Hz}, \mathrm{CH}_{\mathrm{ar}}\right), 7.27-7.39\left(5 \mathrm{H}, \mathrm{m}, 5 \mathrm{xCH}_{\mathrm{ar}}\right) .{ }^{13} \mathrm{C} \mathrm{NMR}\left(\mathrm{CDCl}_{3}\right): \delta 13.9(\mathrm{Me})$, $15.0\left(\mathrm{t}, \mathrm{J}=4.6 \mathrm{~Hz}, \underline{\mathrm{C}} \mathrm{H}_{2} \mathrm{Me}\right), 21.5(\underline{\mathrm{MeCH}}), 24.2(\underline{\mathrm{MeCH}}), 35.9\left(\mathrm{t}, \mathrm{J}=24.2 \mathrm{~Hz}, \underline{\mathrm{CH}_{2}} \mathrm{CF}_{2}\right), 45.4$


$128.7\left(2 \mathrm{xCH}_{\mathrm{ar}}\right), 137.6\left(\mathrm{C}_{\mathrm{ar}, \text { quat }}\right) .{ }^{19} \mathrm{~F} \mathrm{NMR}\left(\mathrm{CDCl}_{3}\right): \delta-106.9(2 \mathrm{~F}, \mathrm{~m}) . \mathrm{IR}(\mathrm{NaCl}): v_{\max } 3339$ $\mathrm{cm}^{-1}$. MS (ES+): m/z(\%): $241\left(\mathrm{M}+\mathrm{H}^{+}, 100\right)$. Copies of ${ }^{1} \mathrm{H}$ NMR and ${ }^{13} \mathrm{C}$ NMR spectra are included in chapter III of the supporting information file. 
III. Copies of ${ }^{1} \mathrm{H}$ NMR and ${ }^{13} \mathrm{C}$ NMR spectra of compounds $2 \mathbf{e}, \mathbf{2 g}, \mathbf{2 i}, \mathbf{3 a - c}, \mathbf{3 f}$, 3g, 4a-e, 5a-c, 6b and 8a-c 












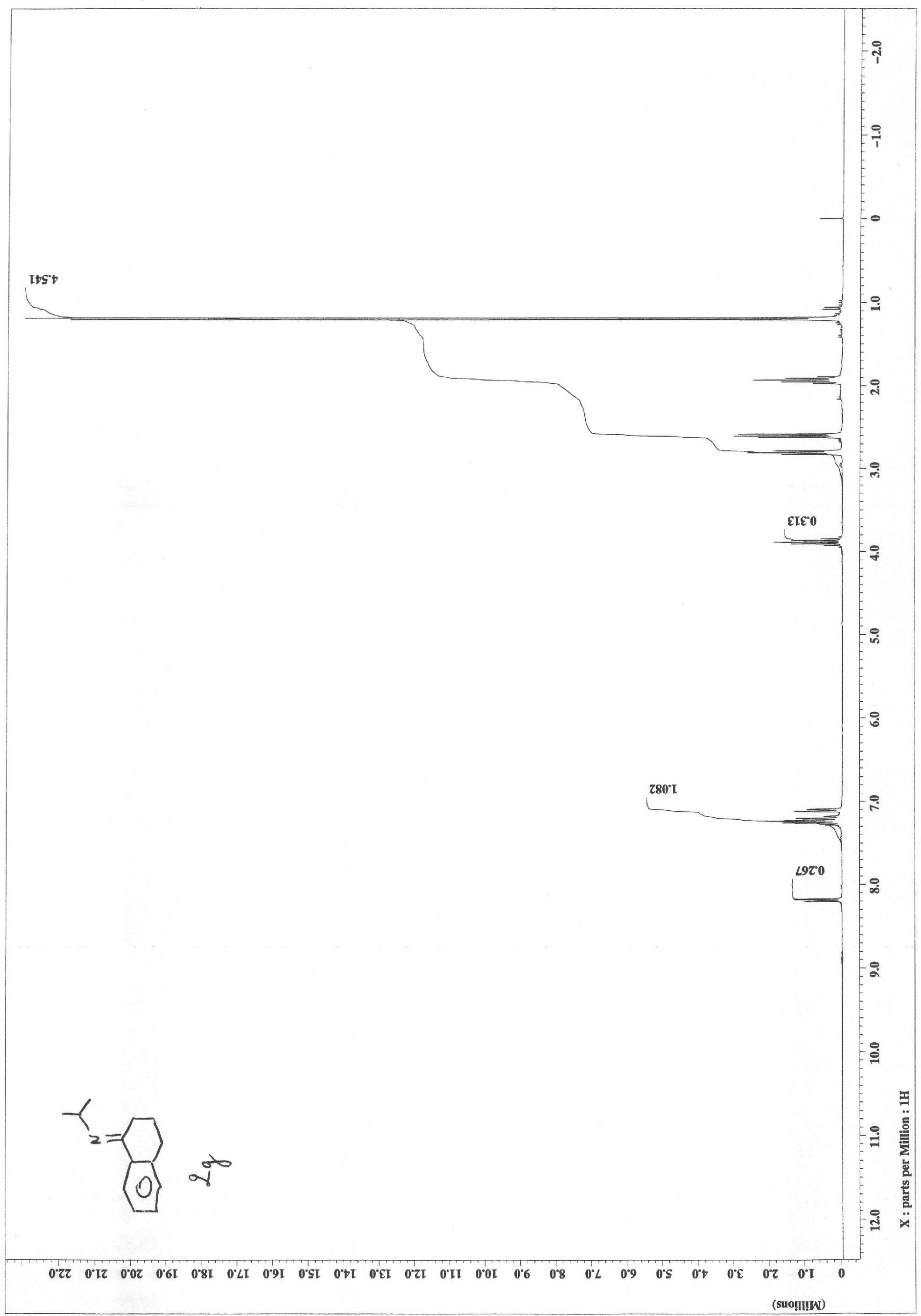









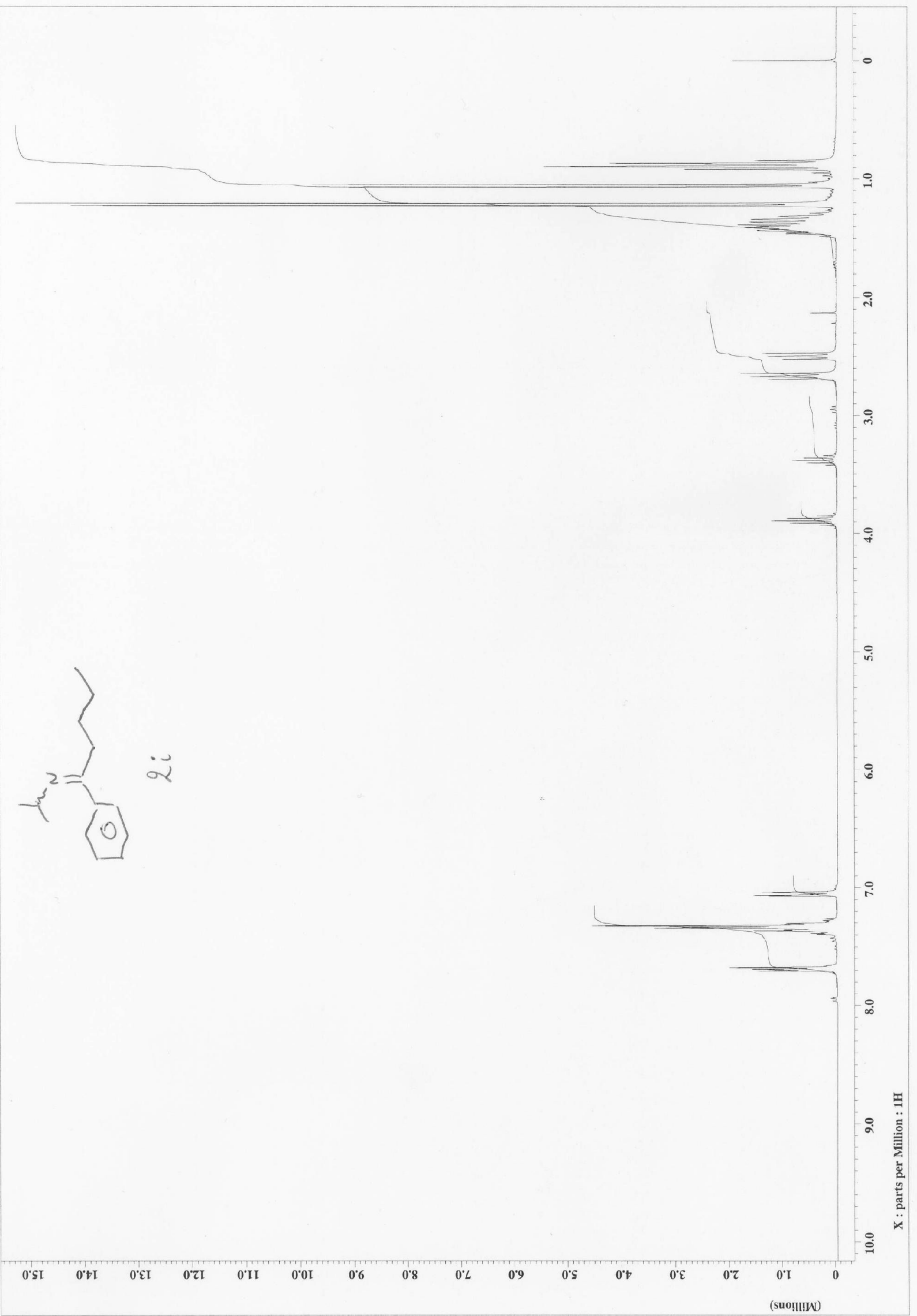




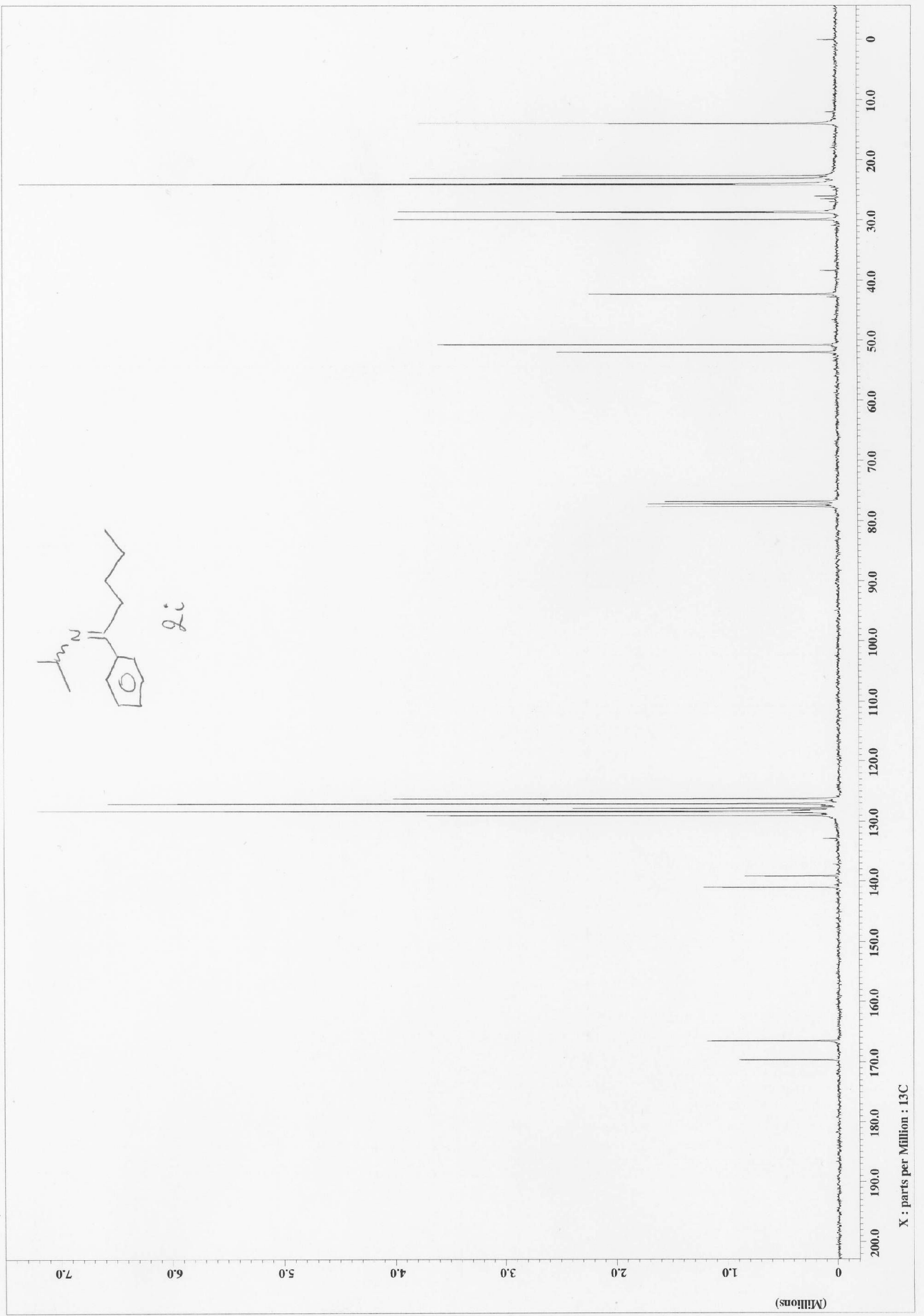




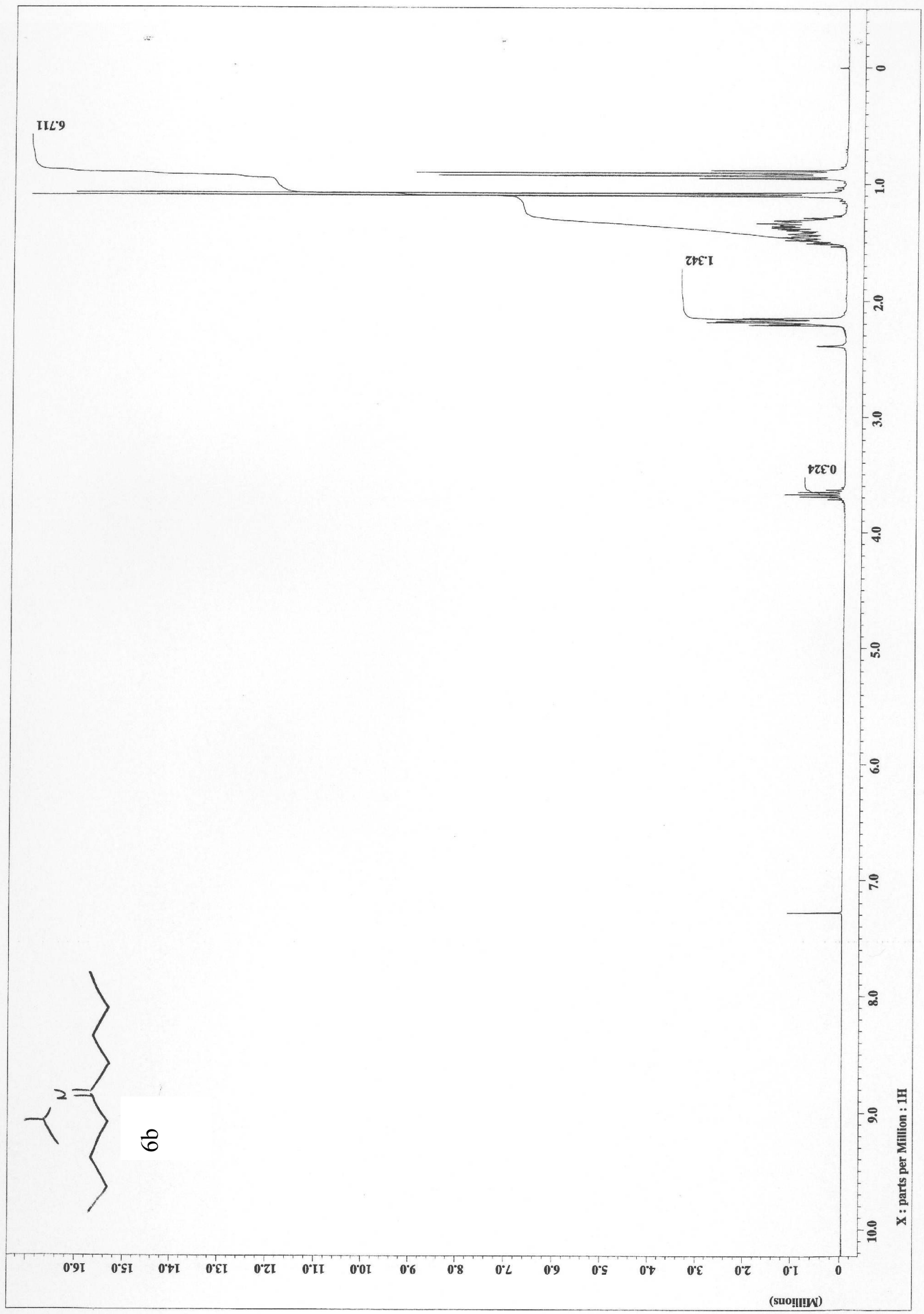




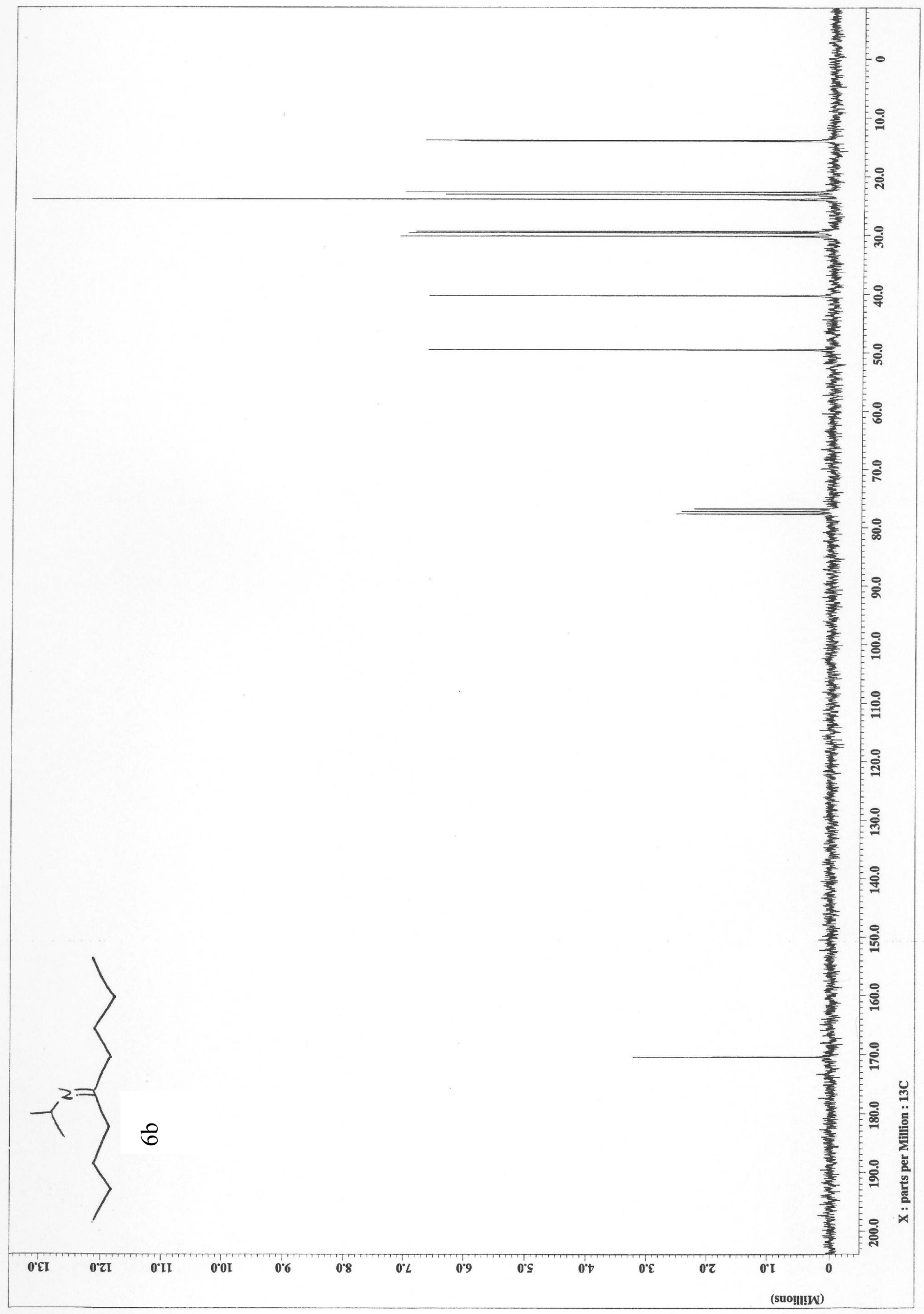




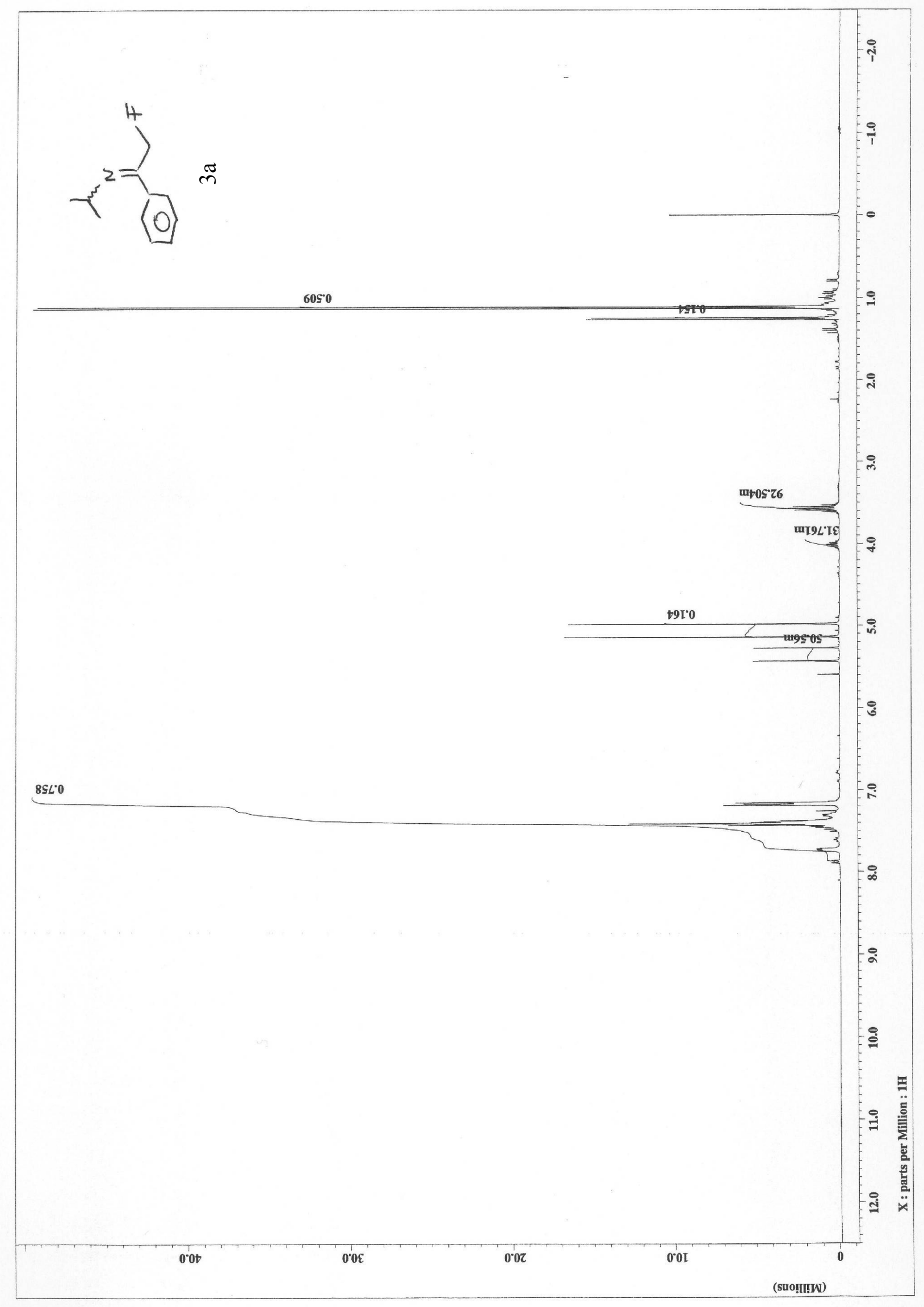









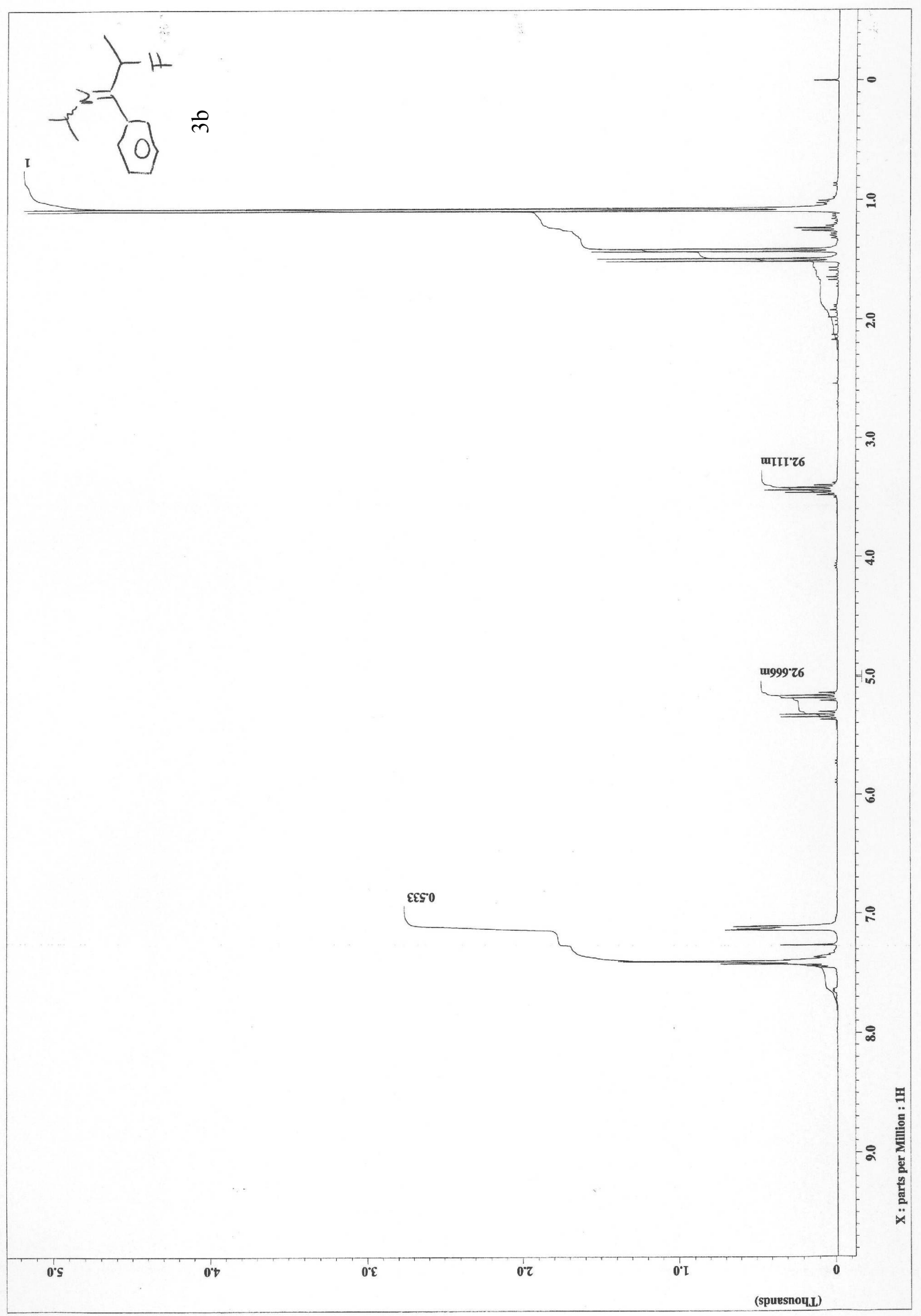




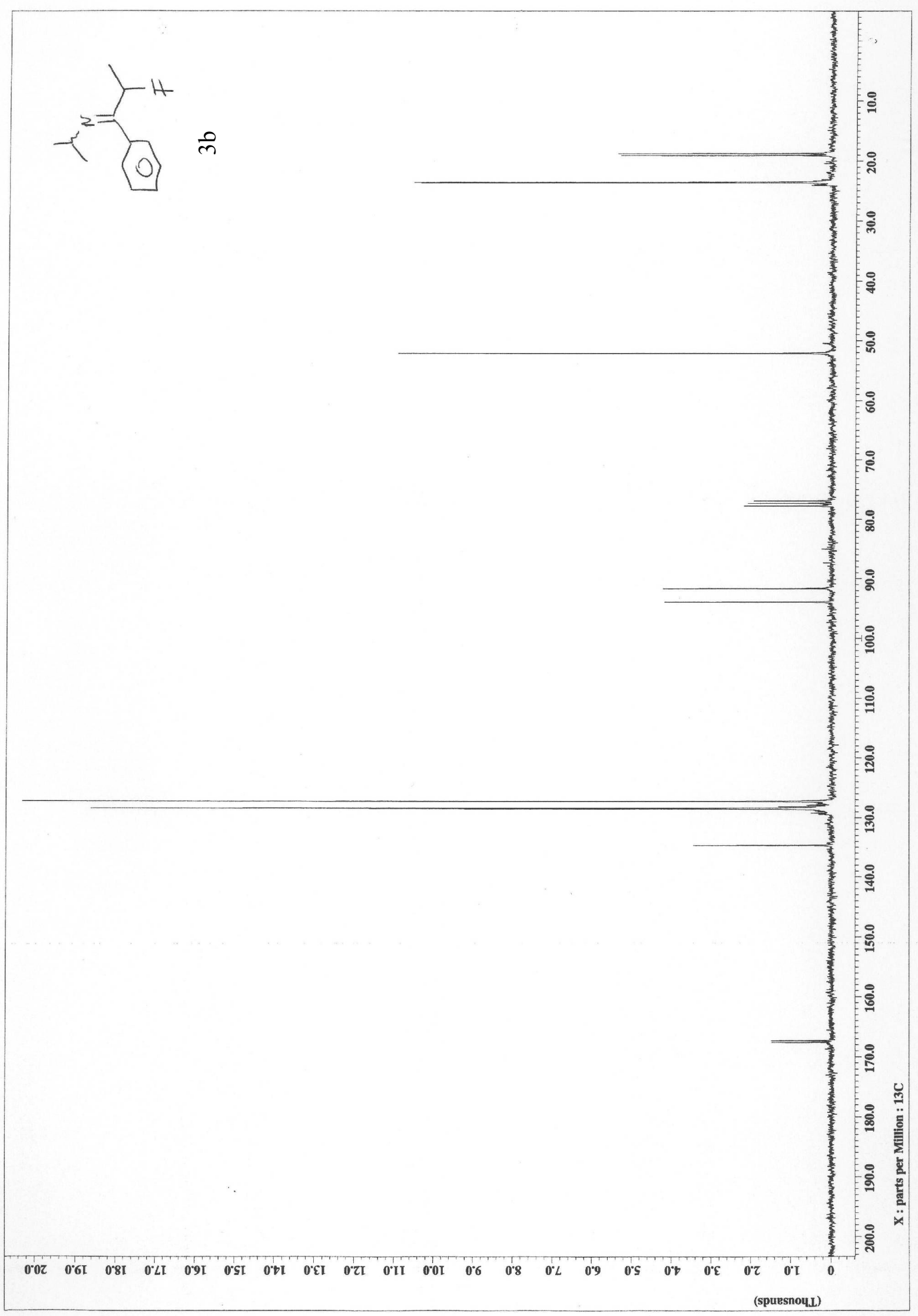




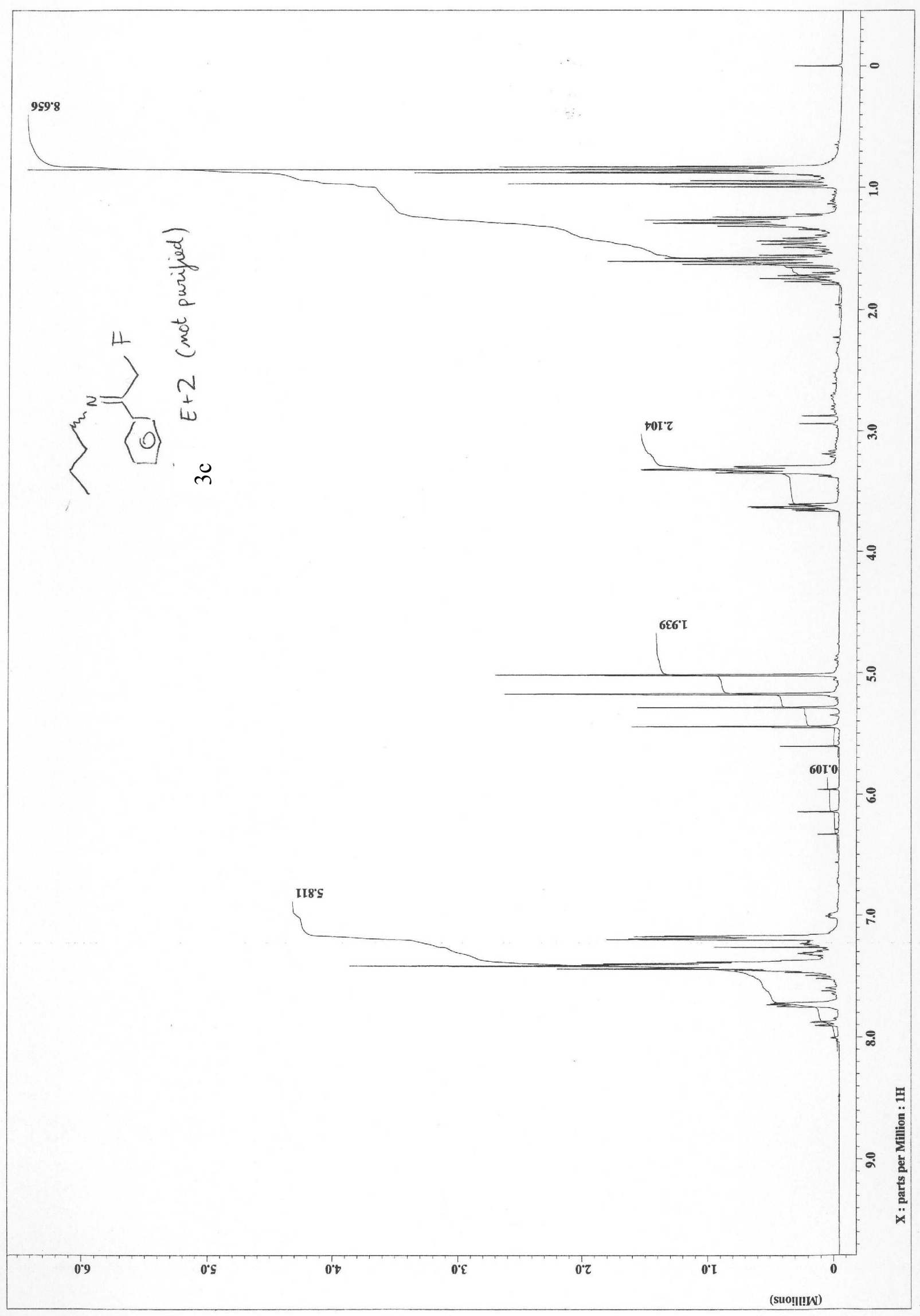




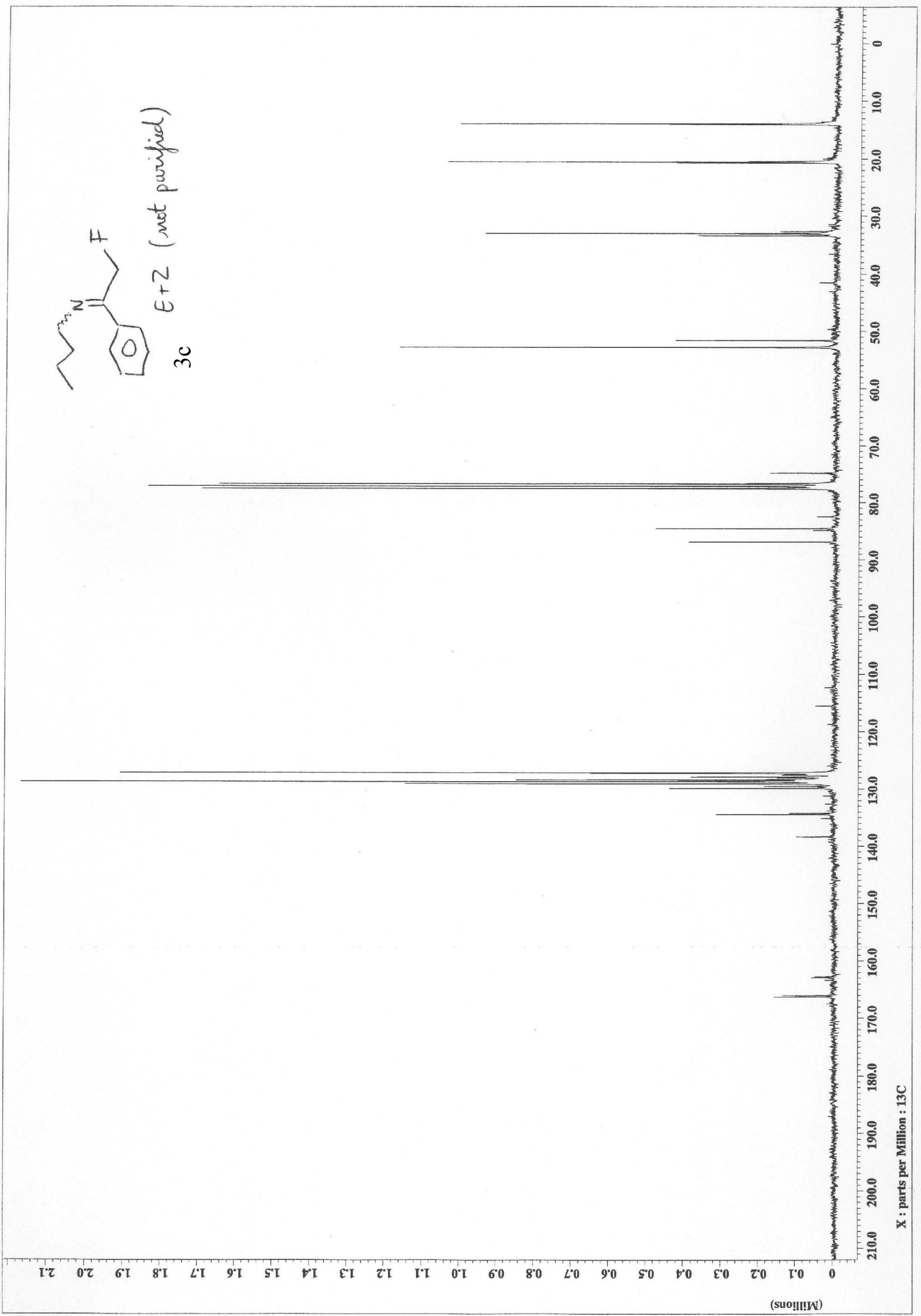




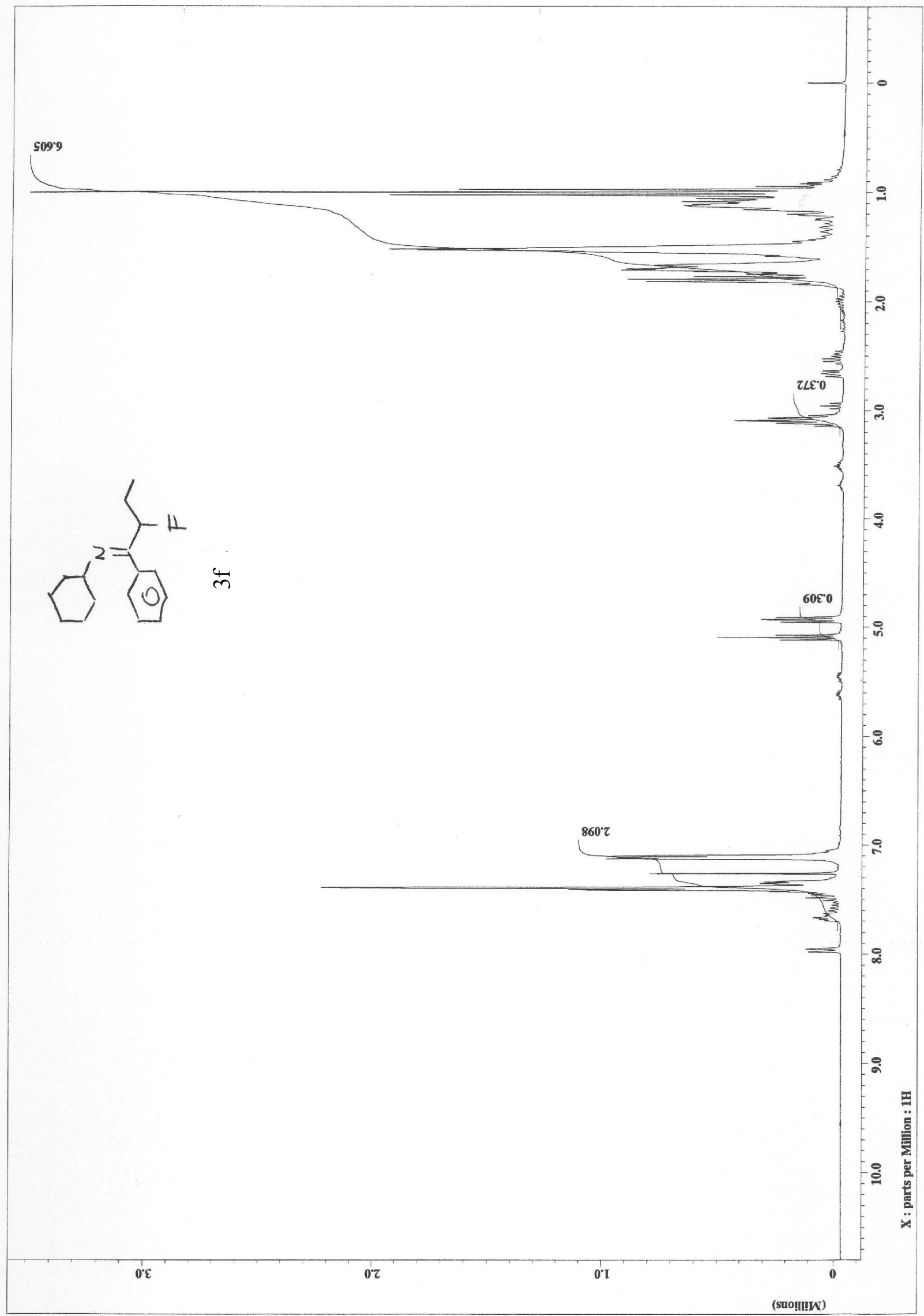




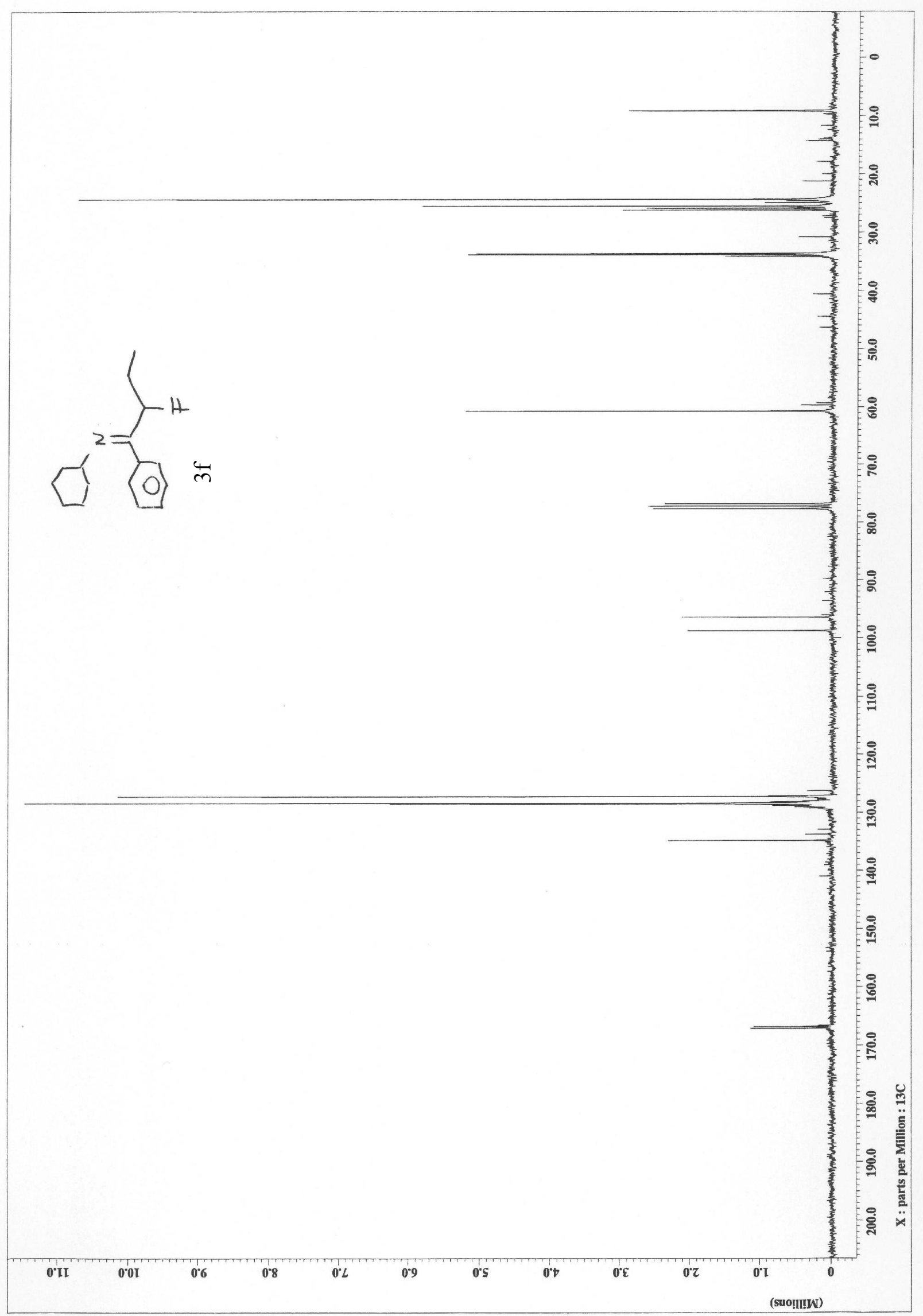




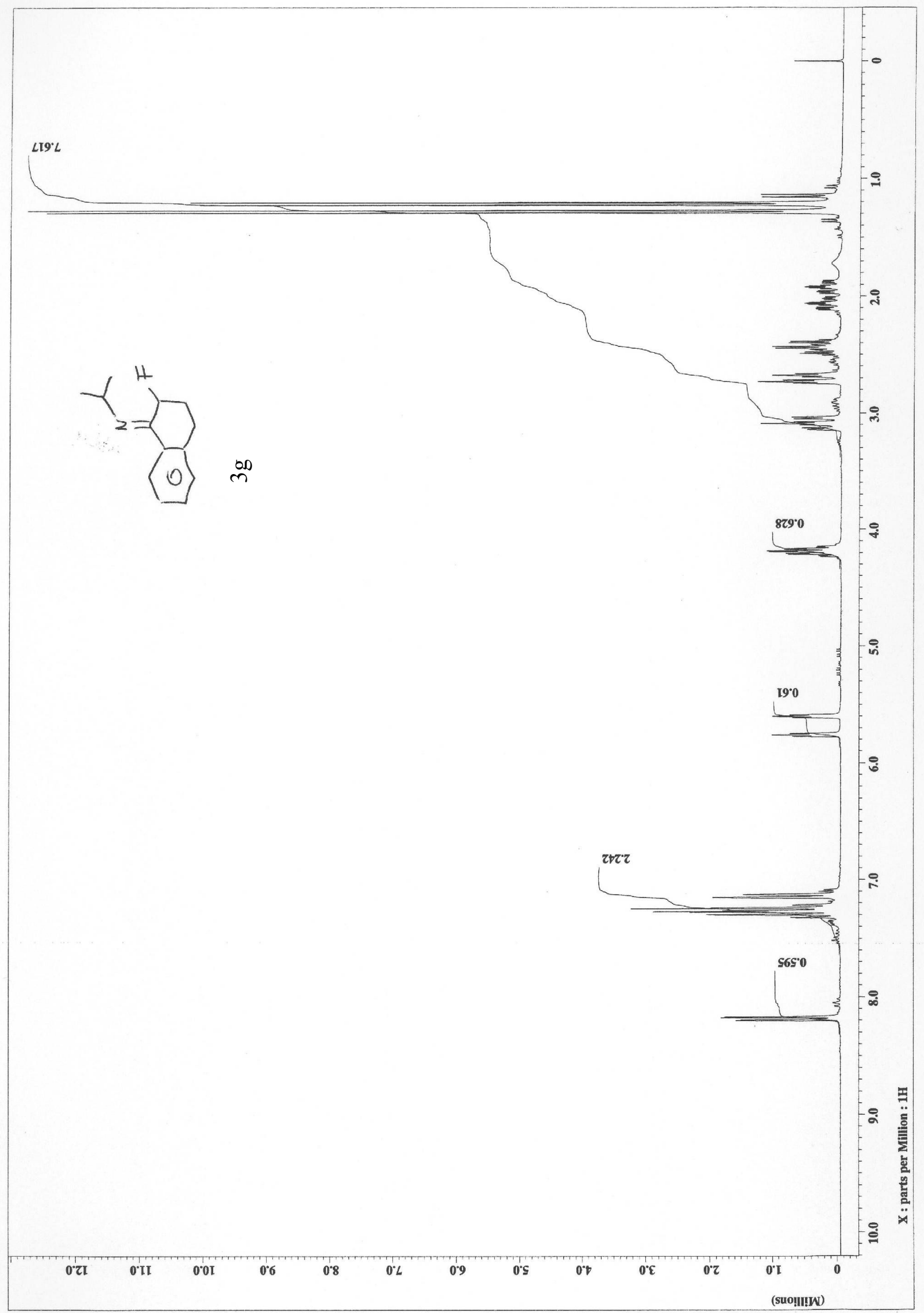




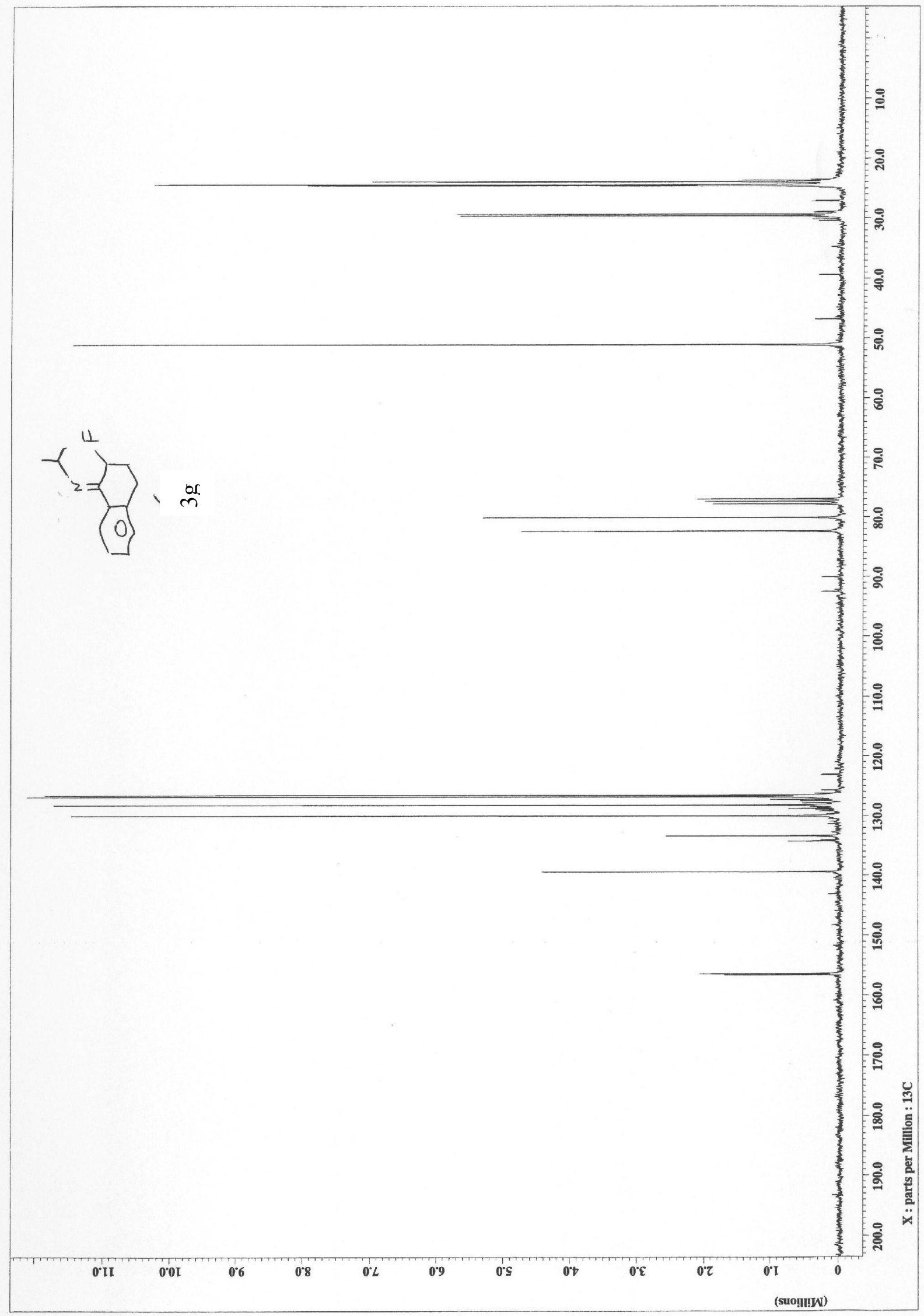


S-32

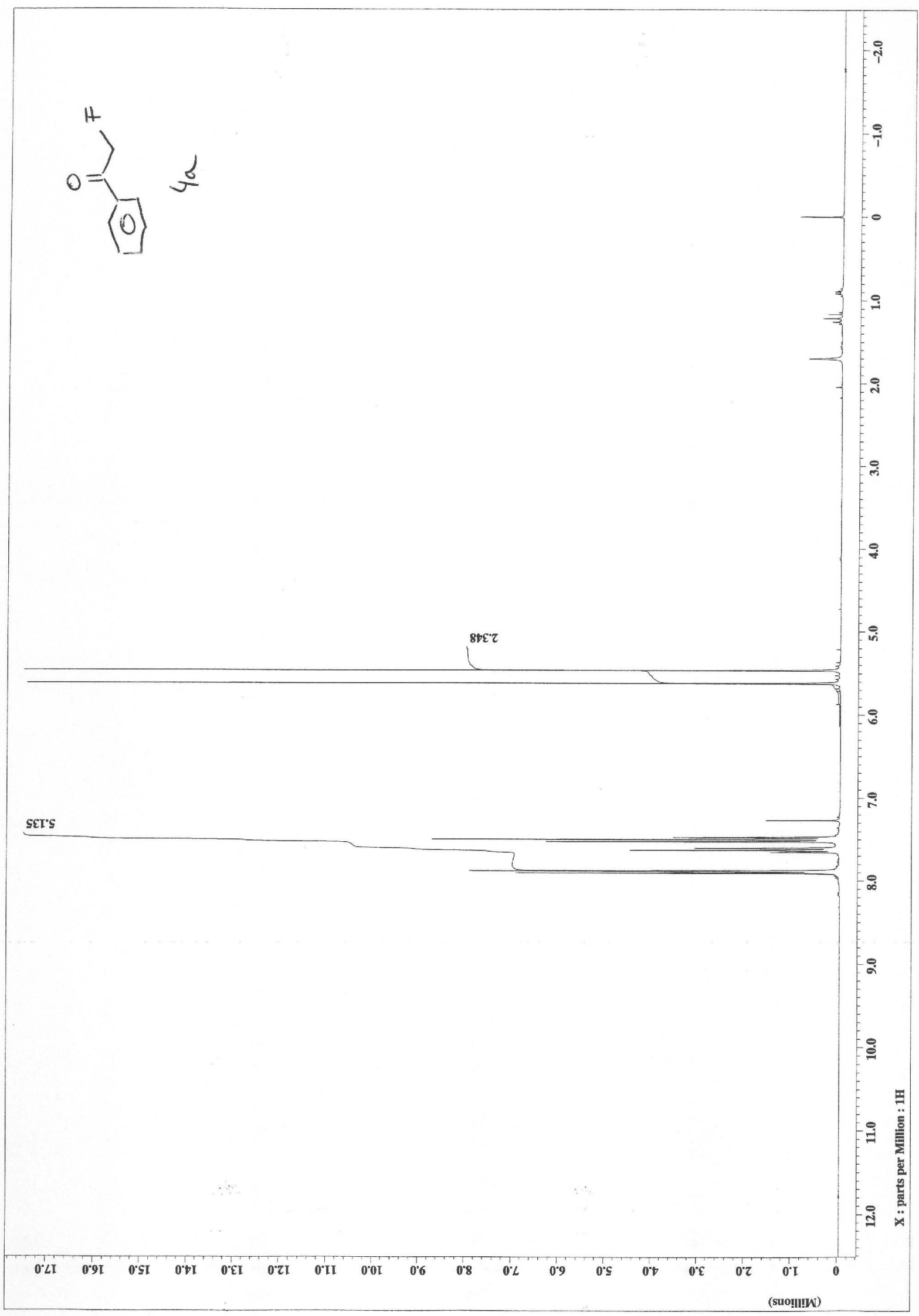




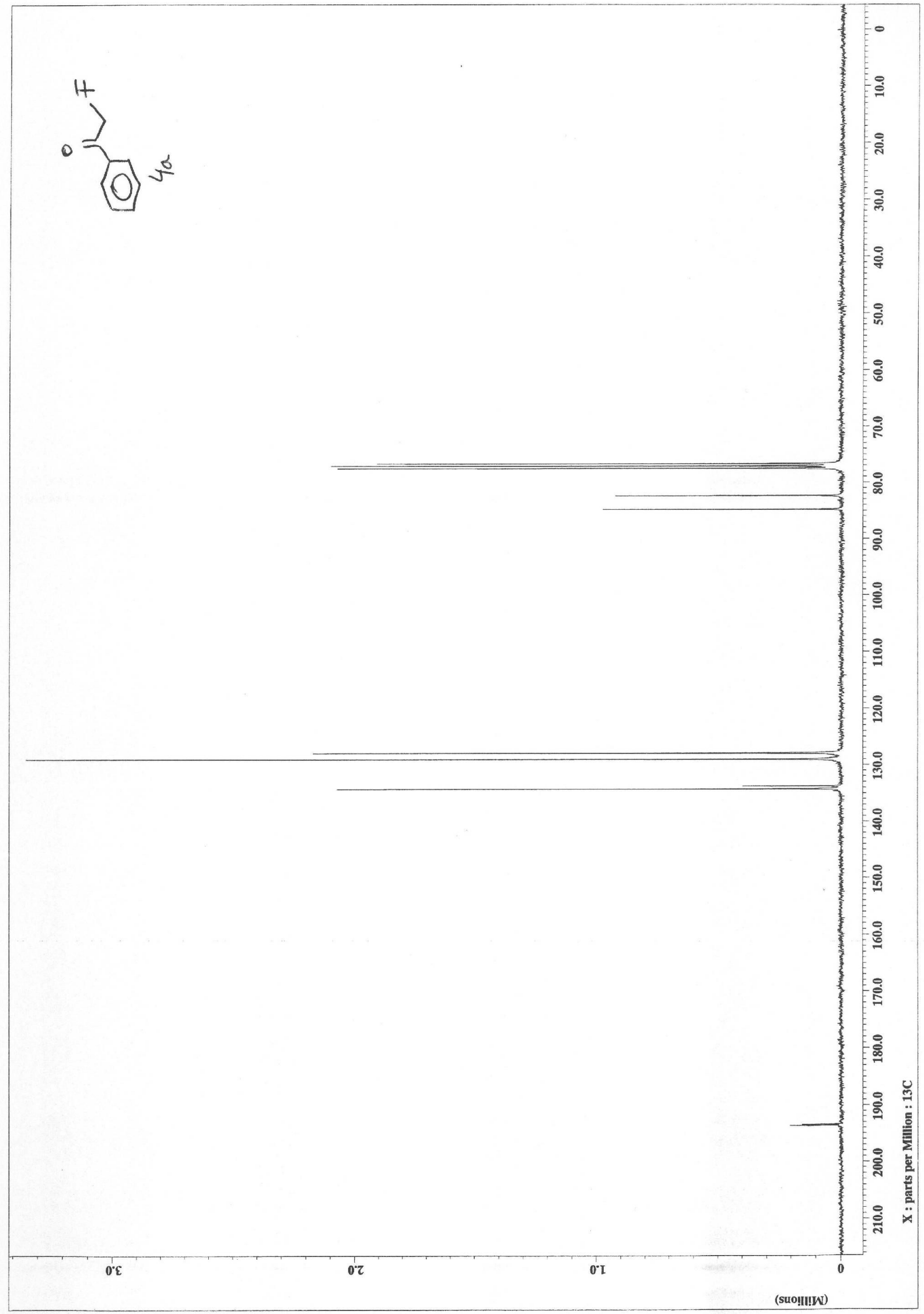




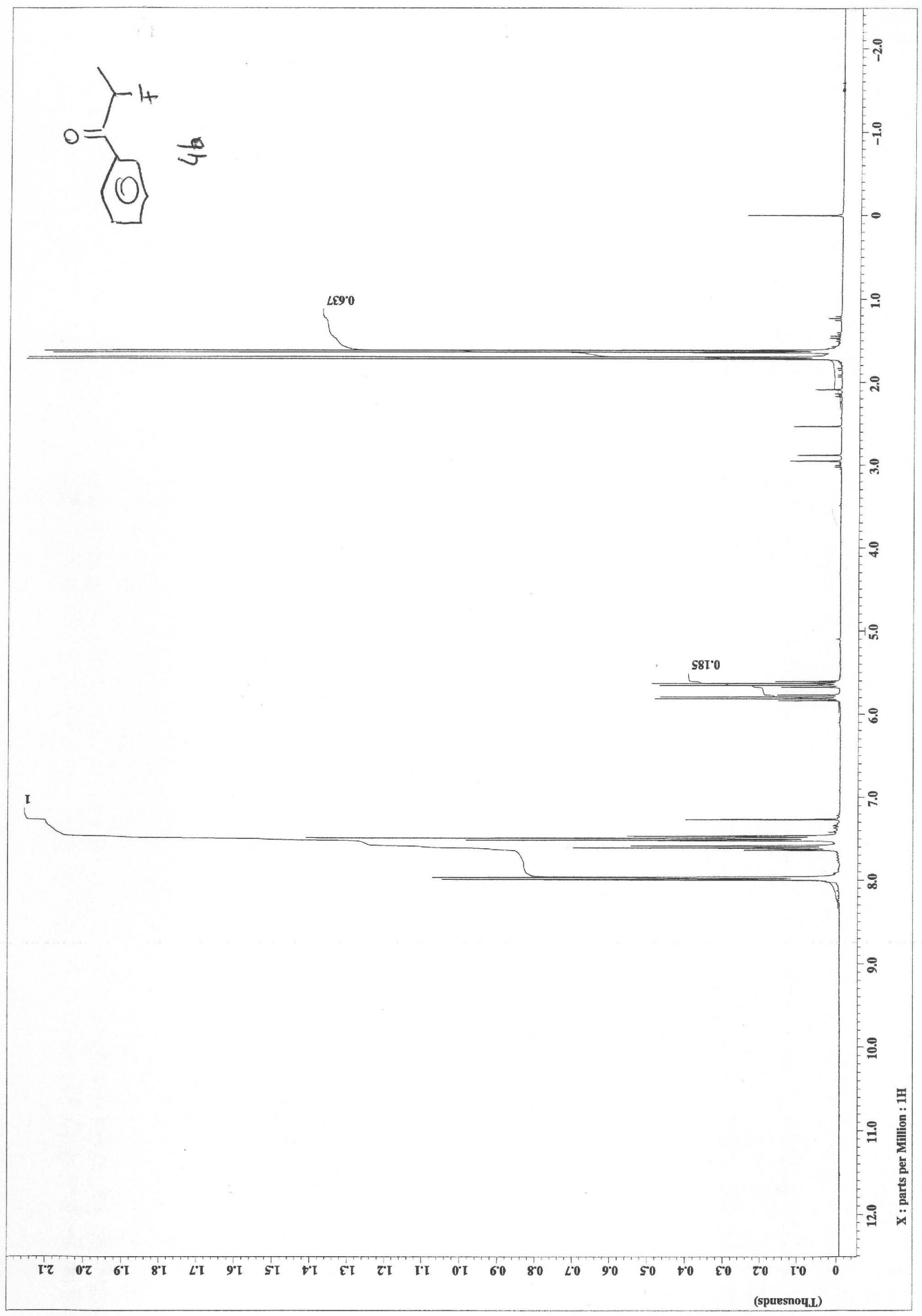









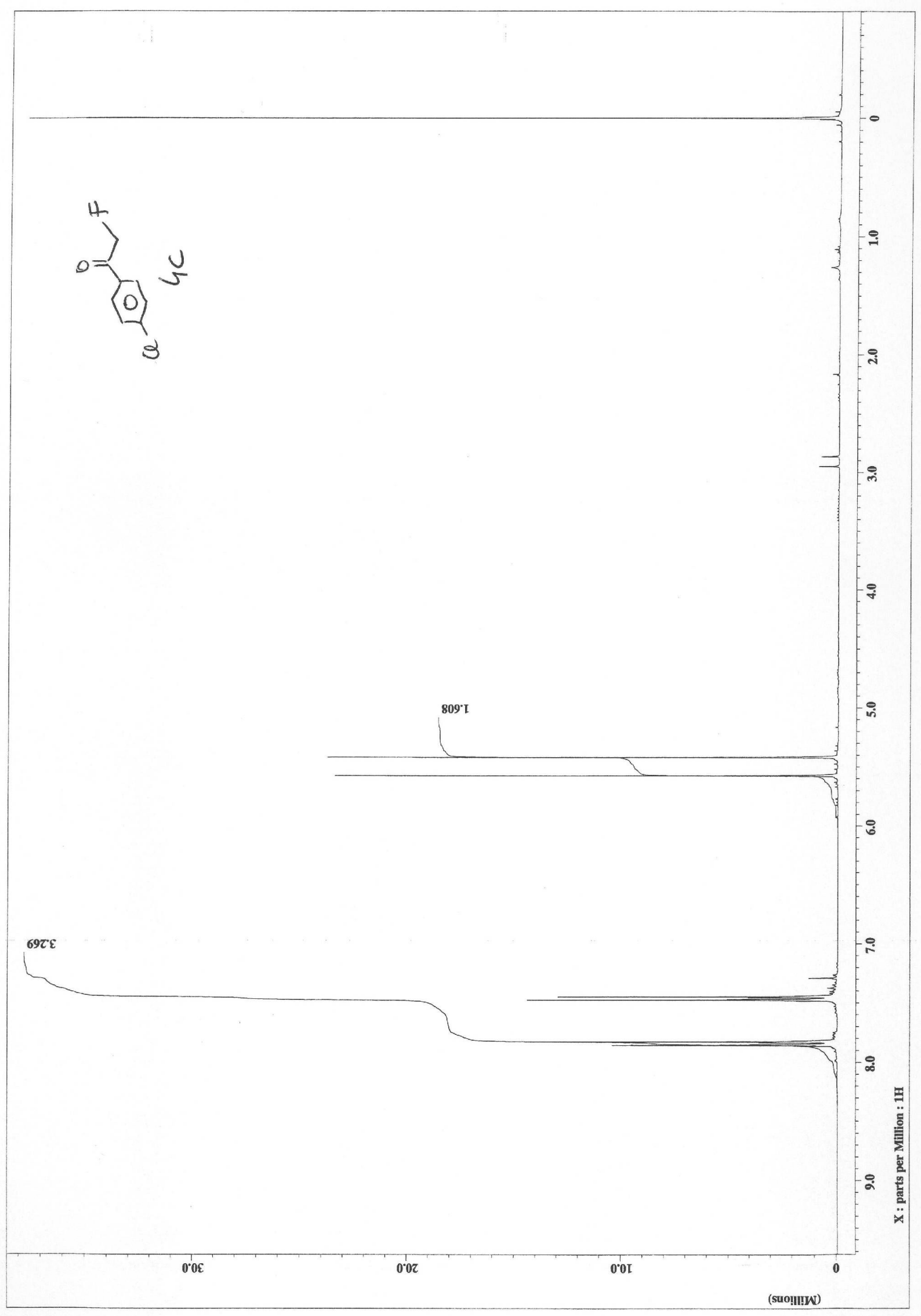




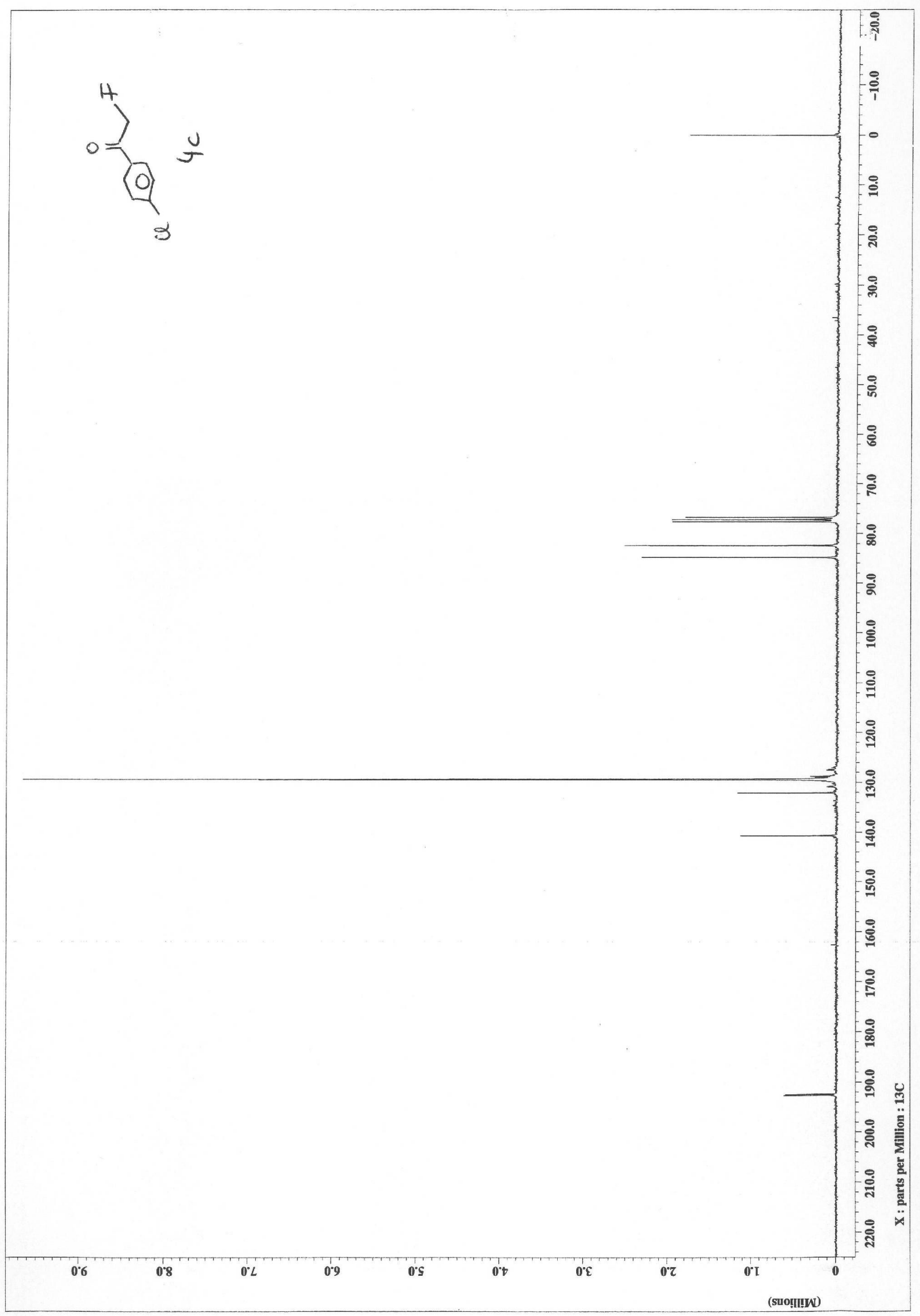







S-39

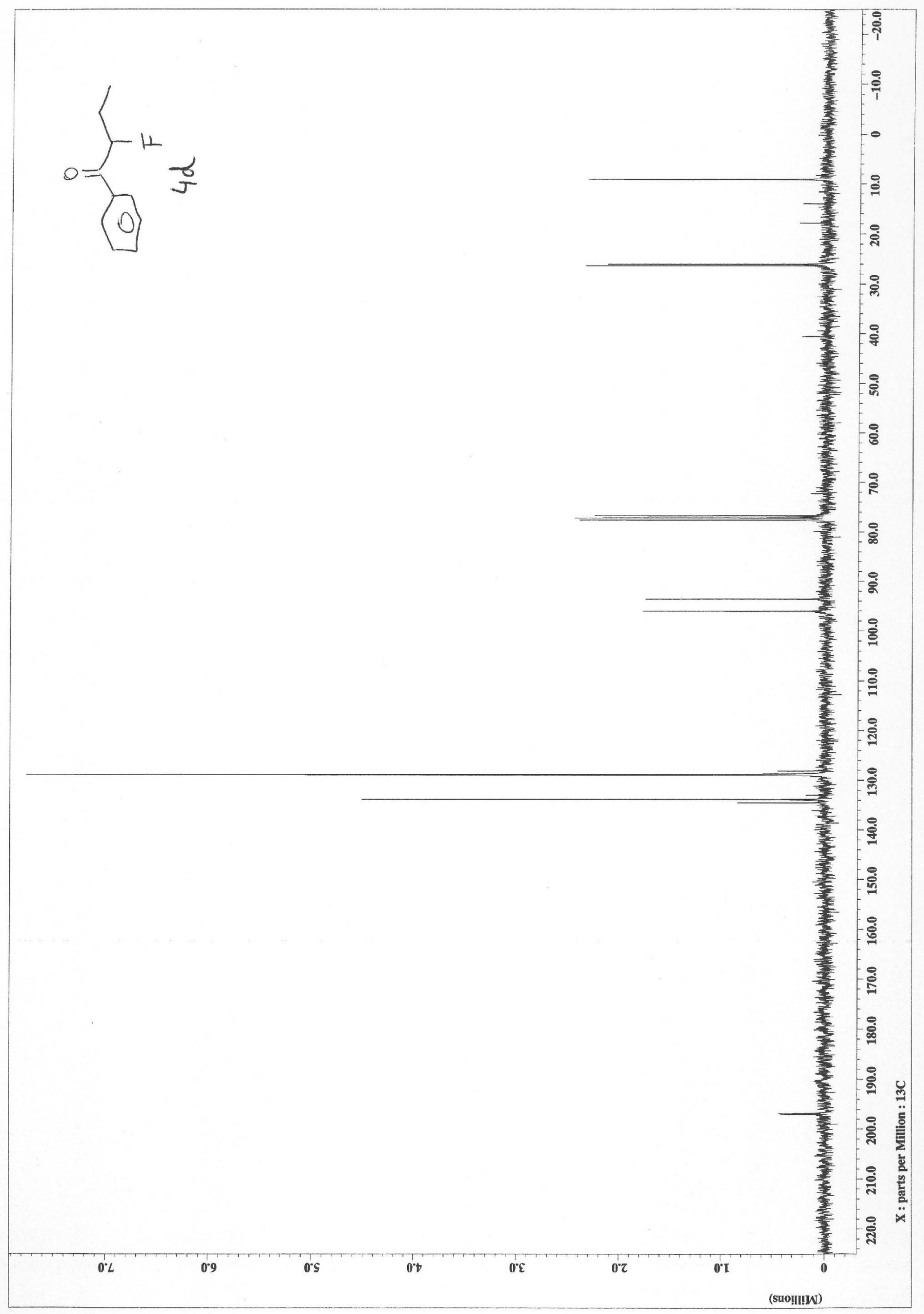




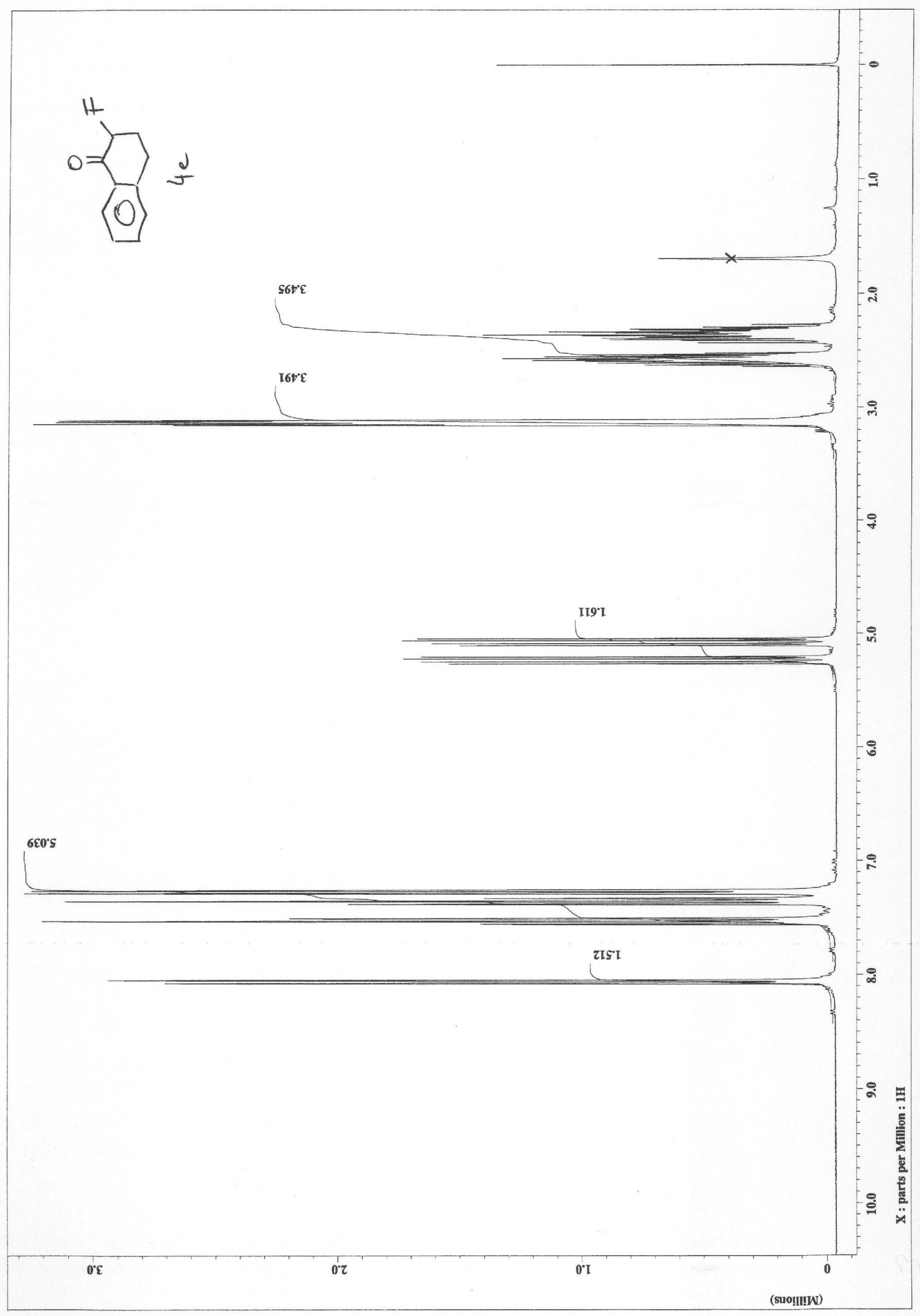




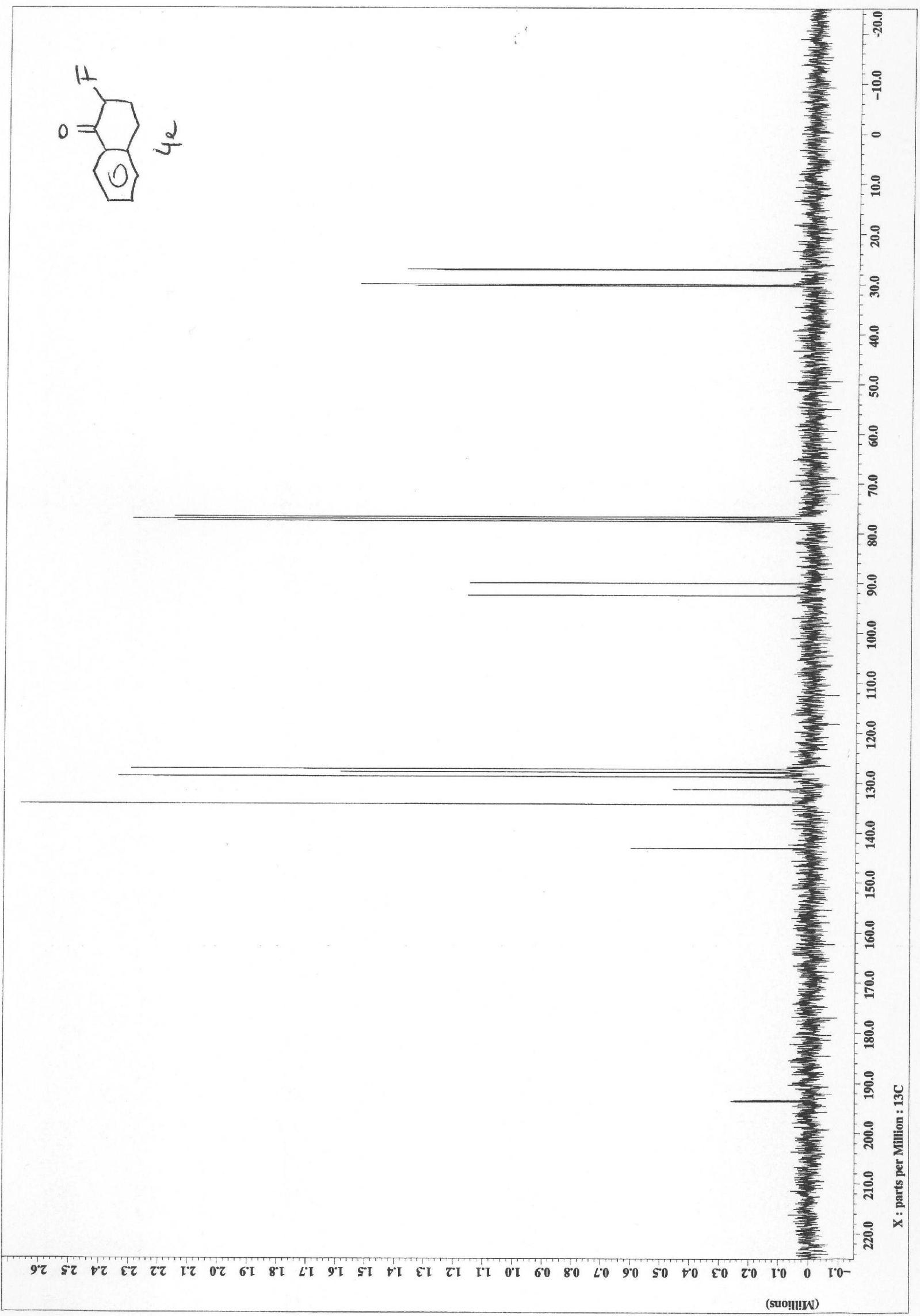




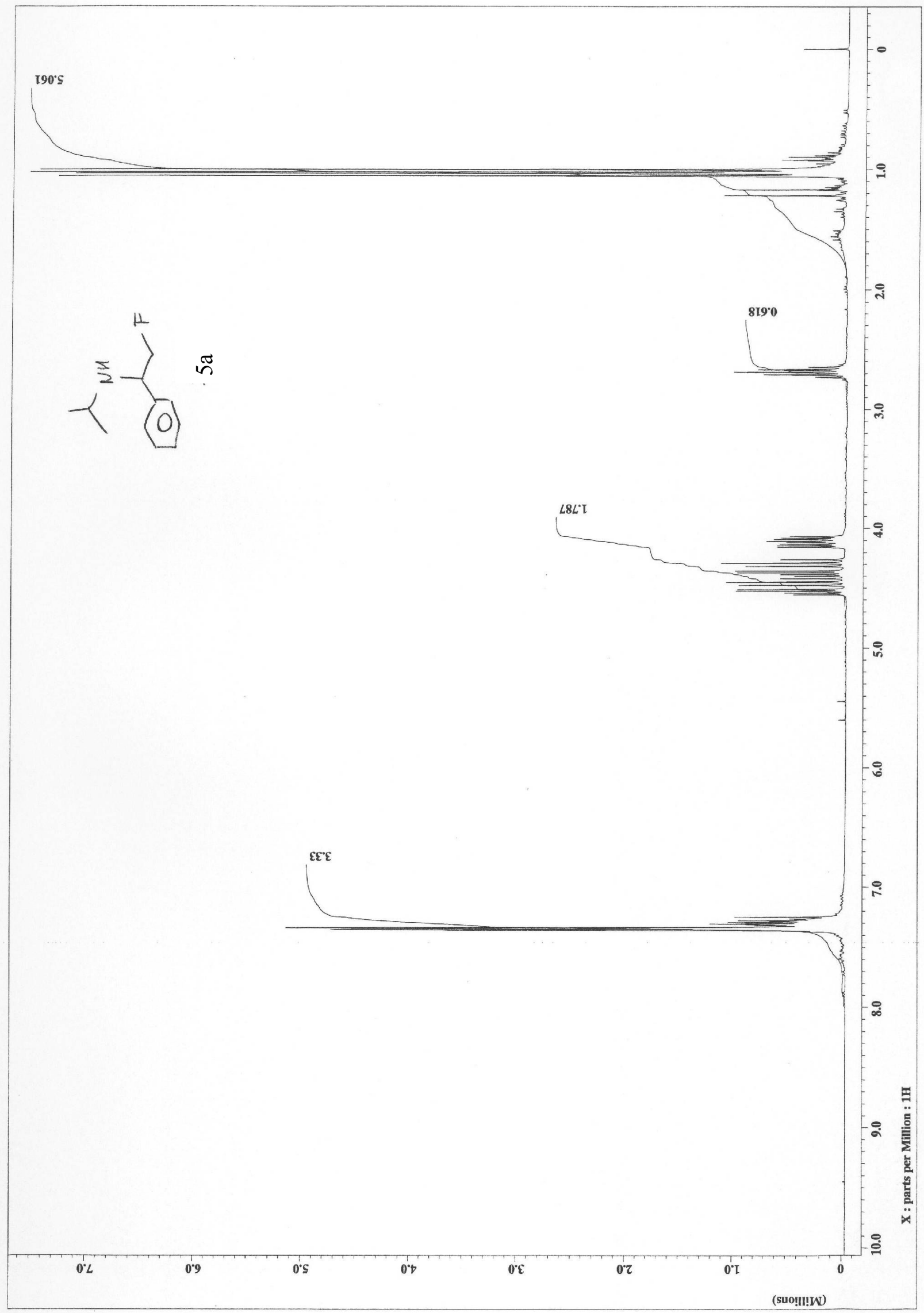




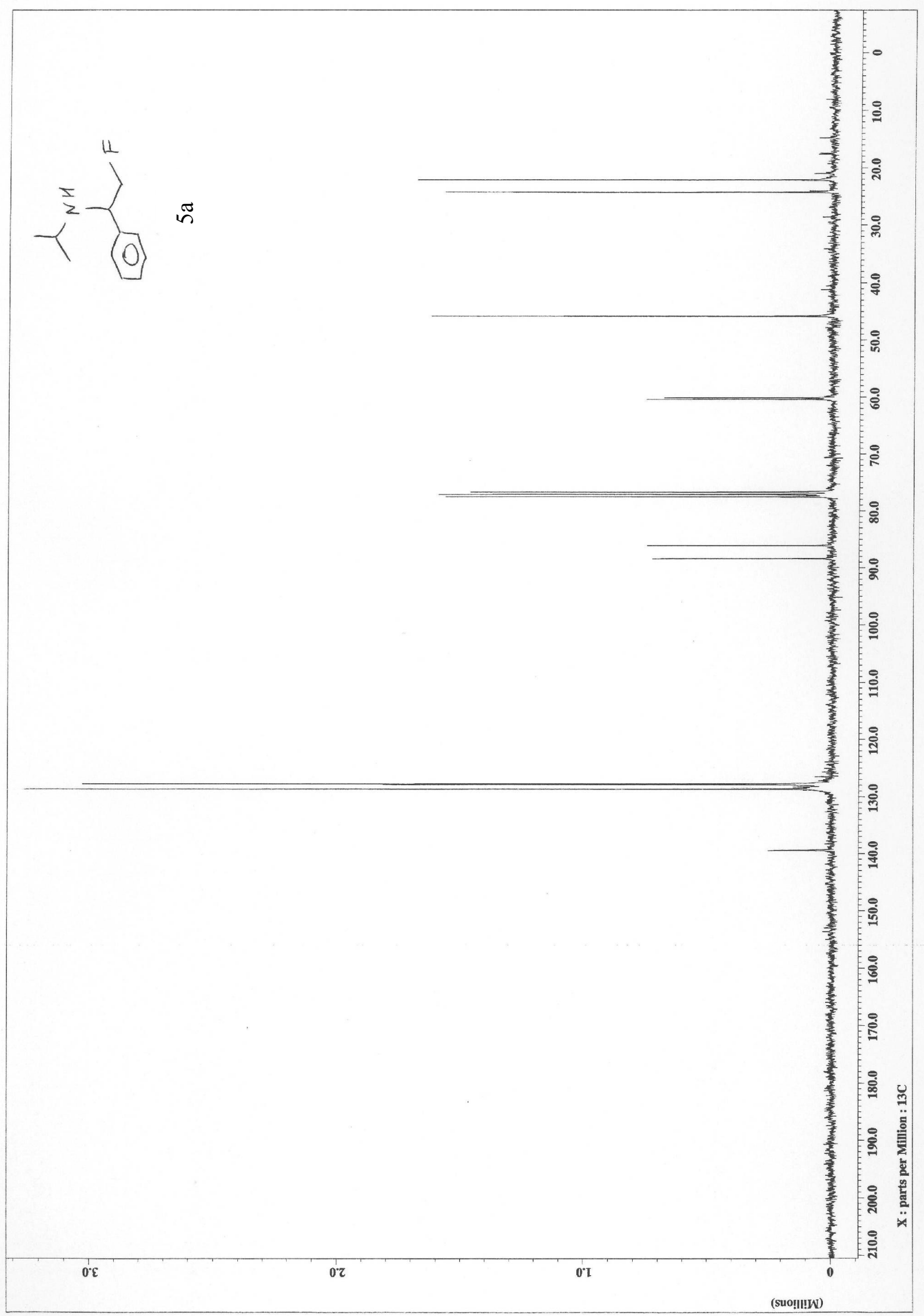




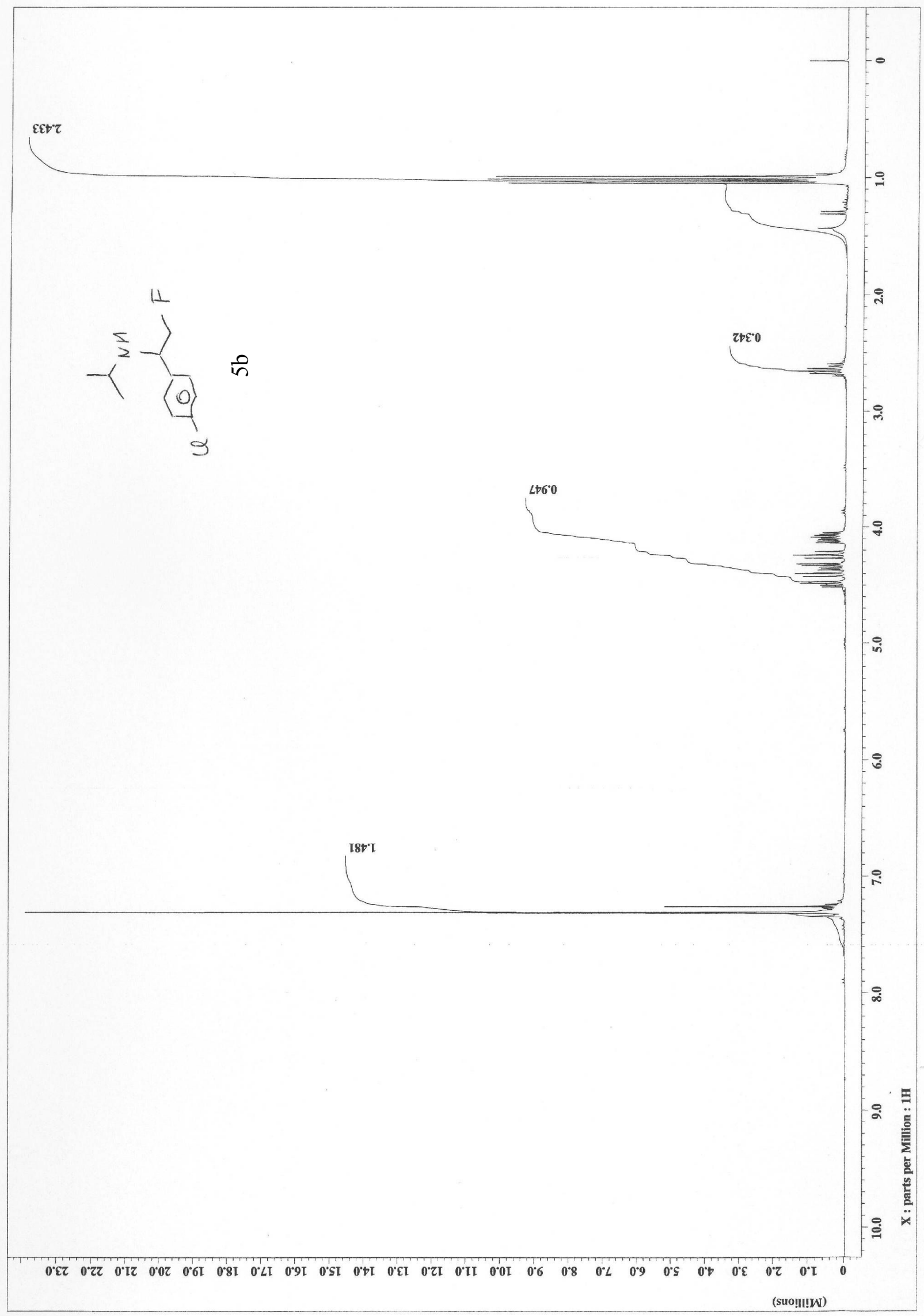









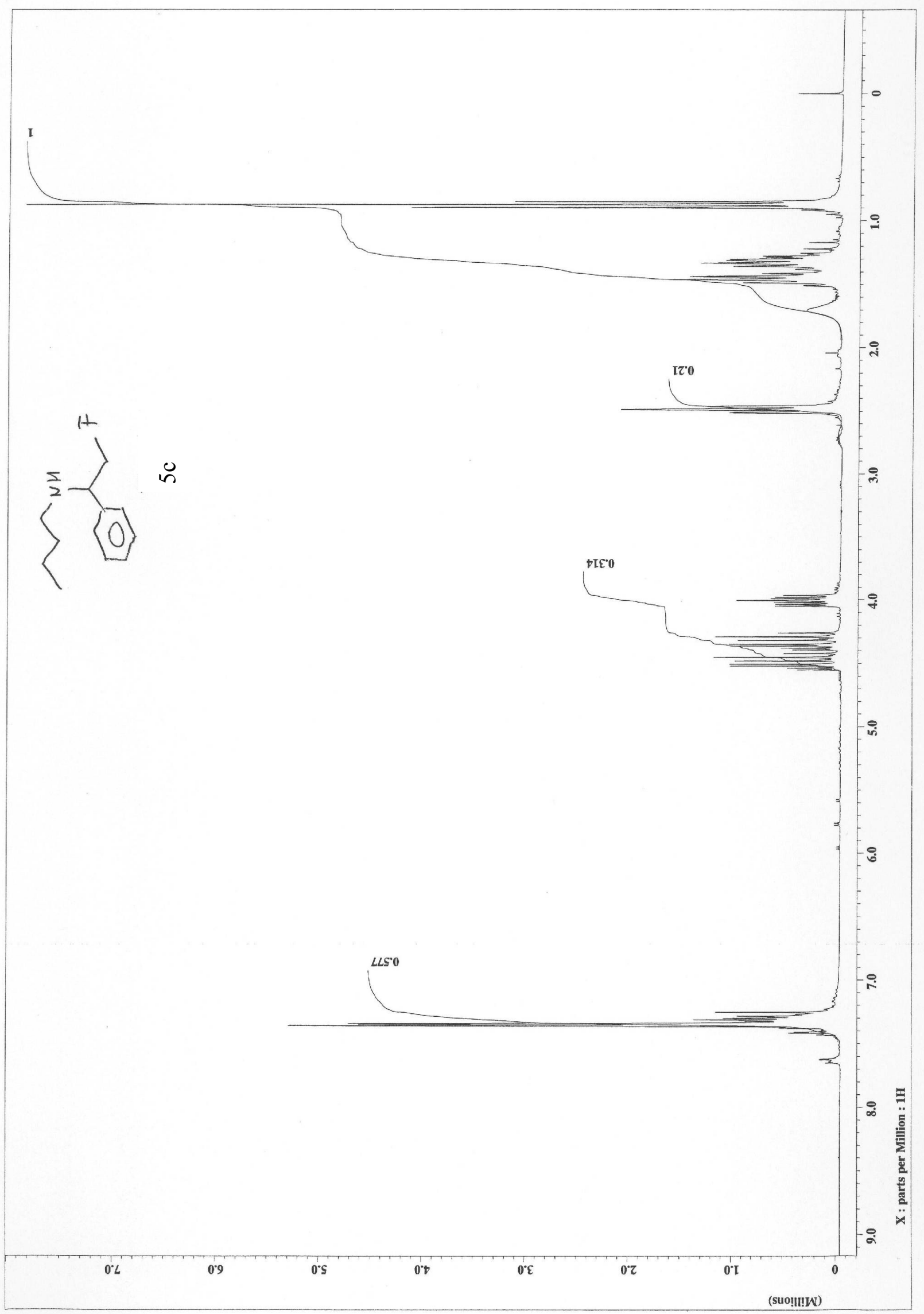









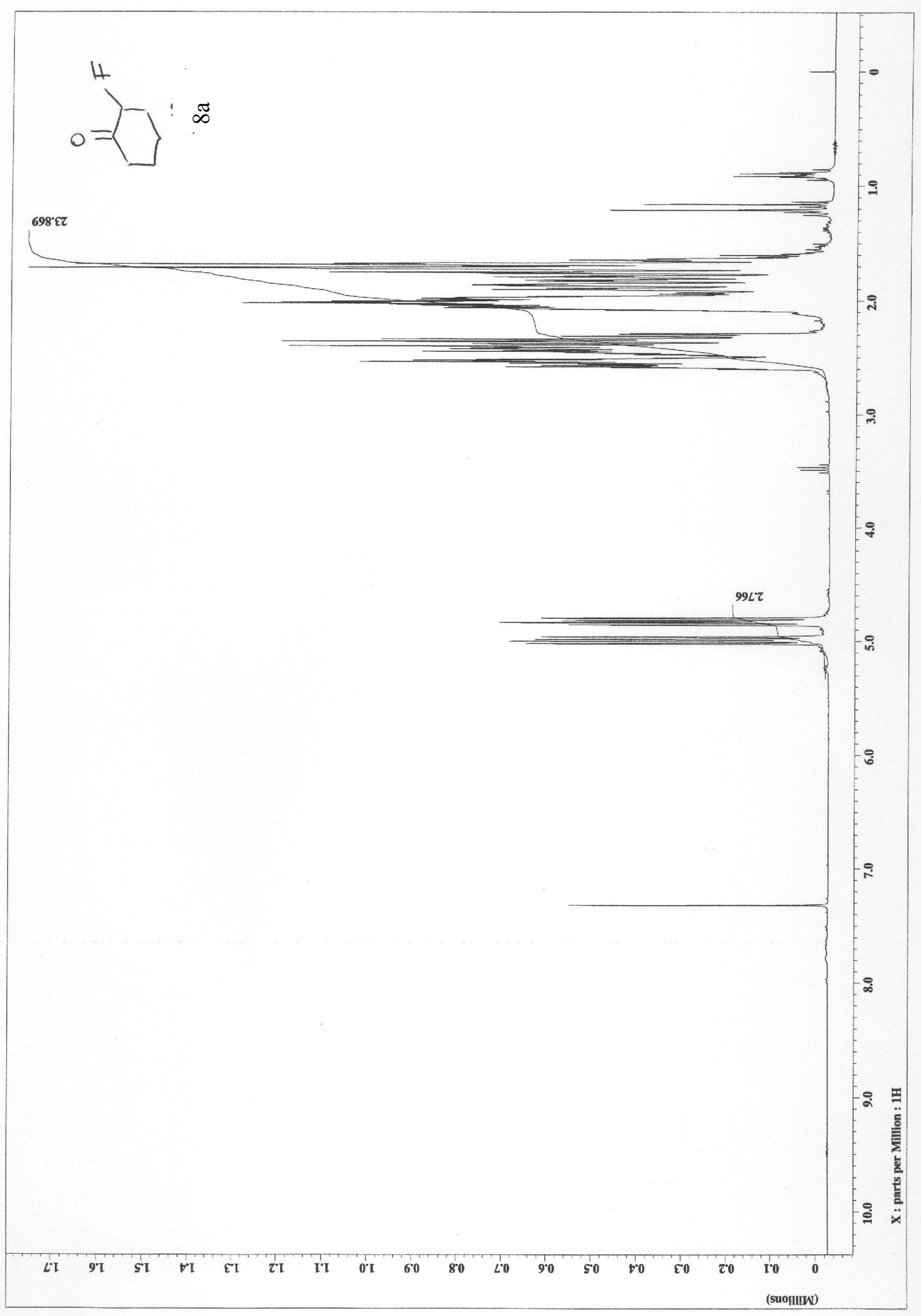




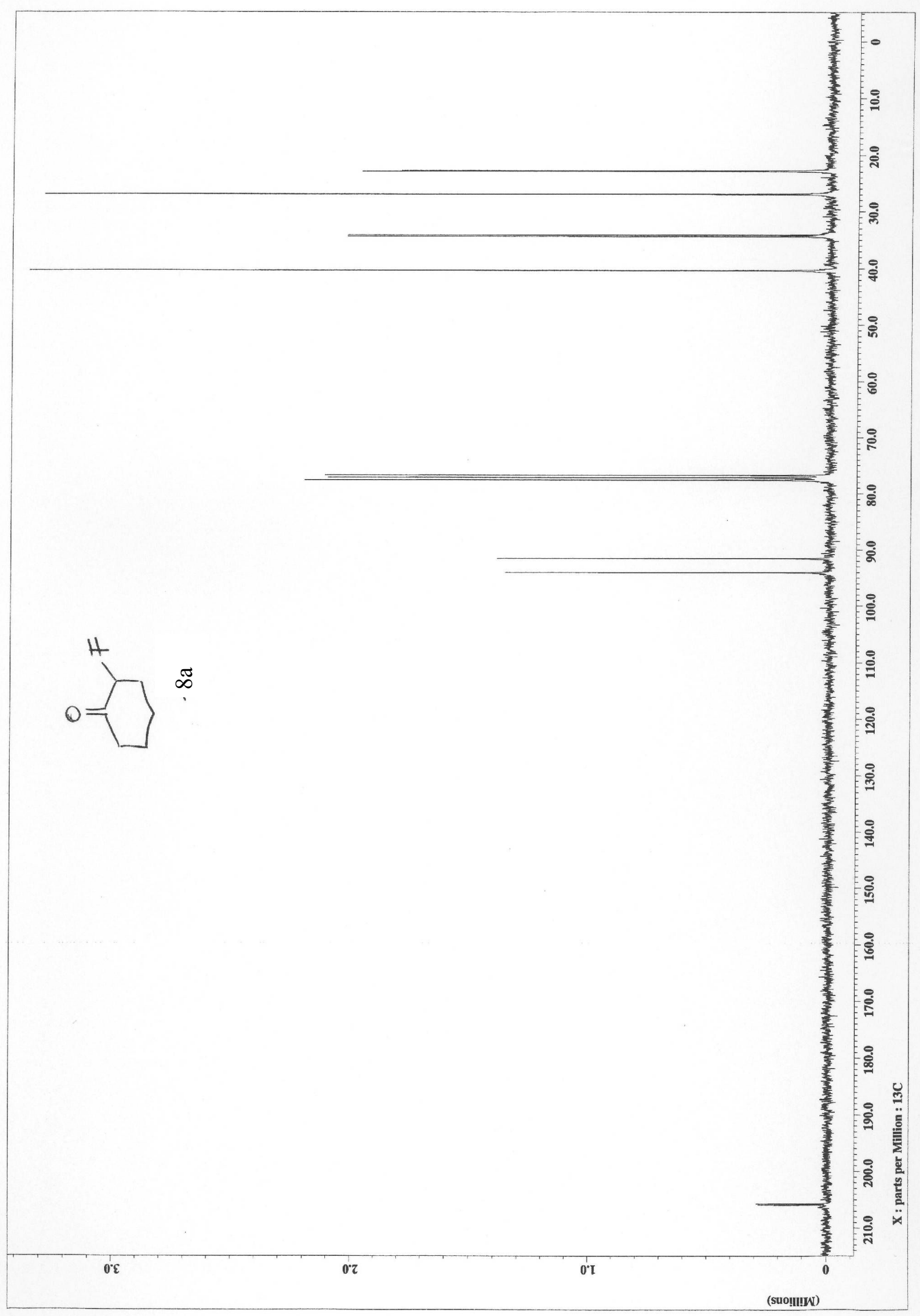




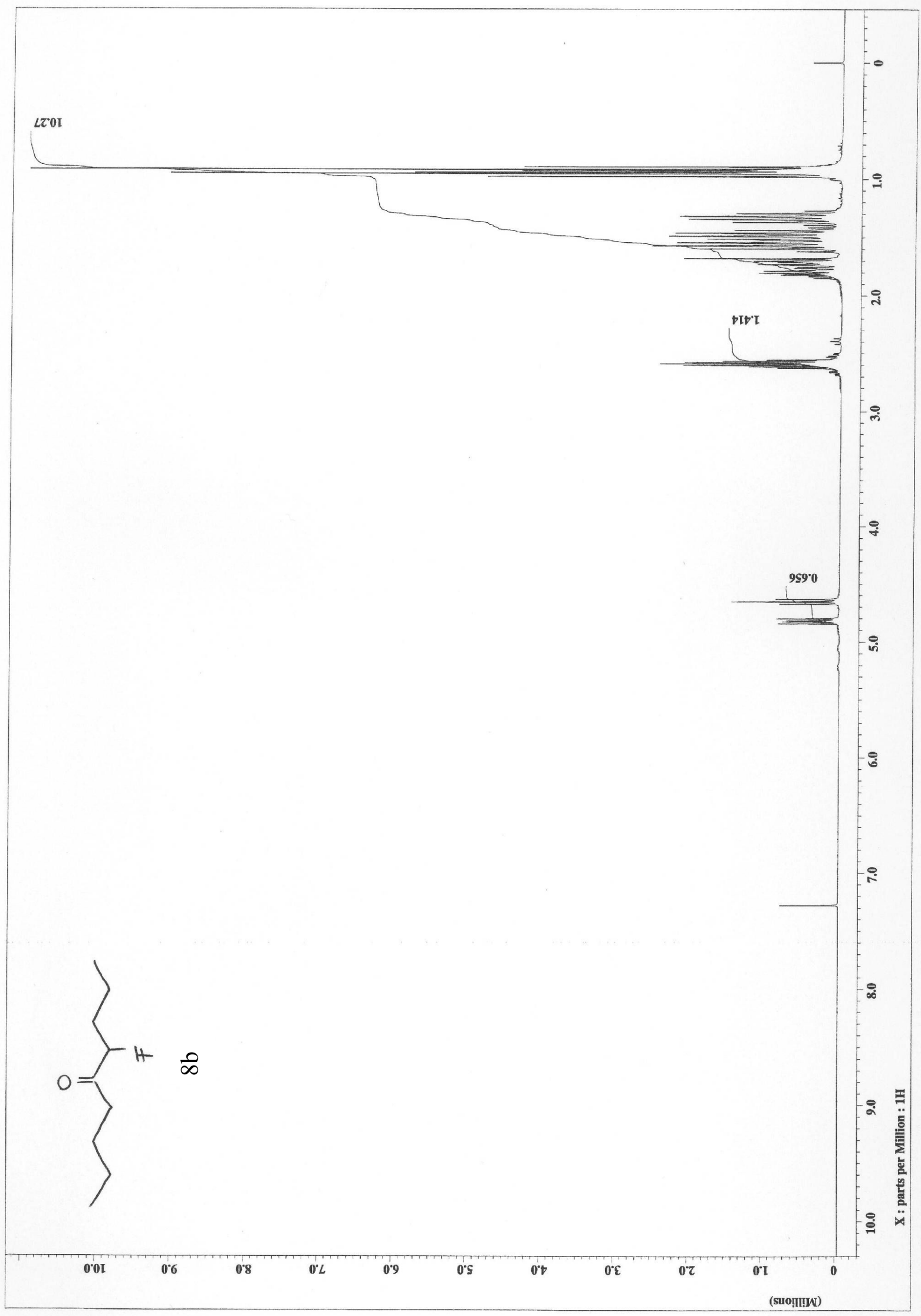




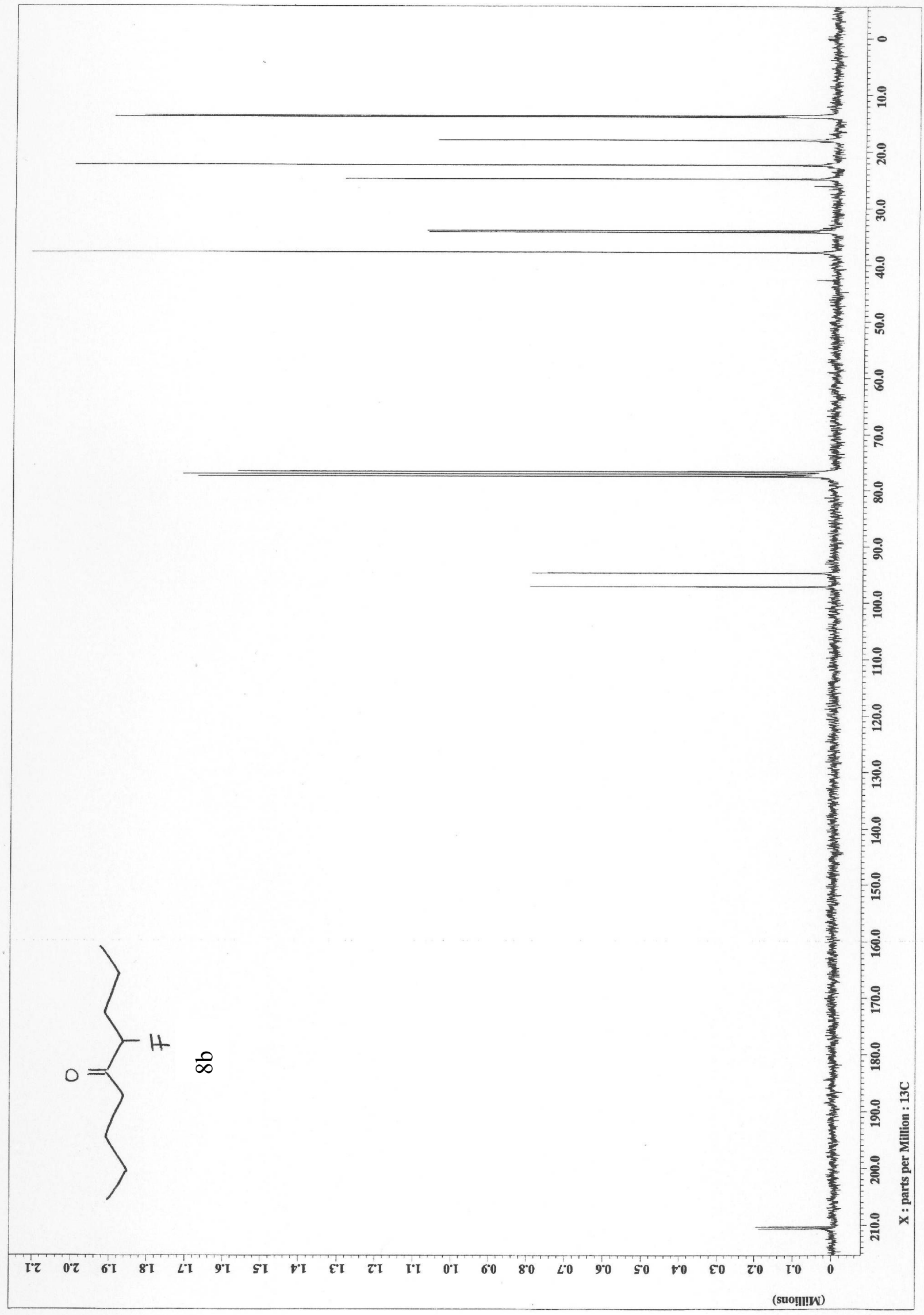




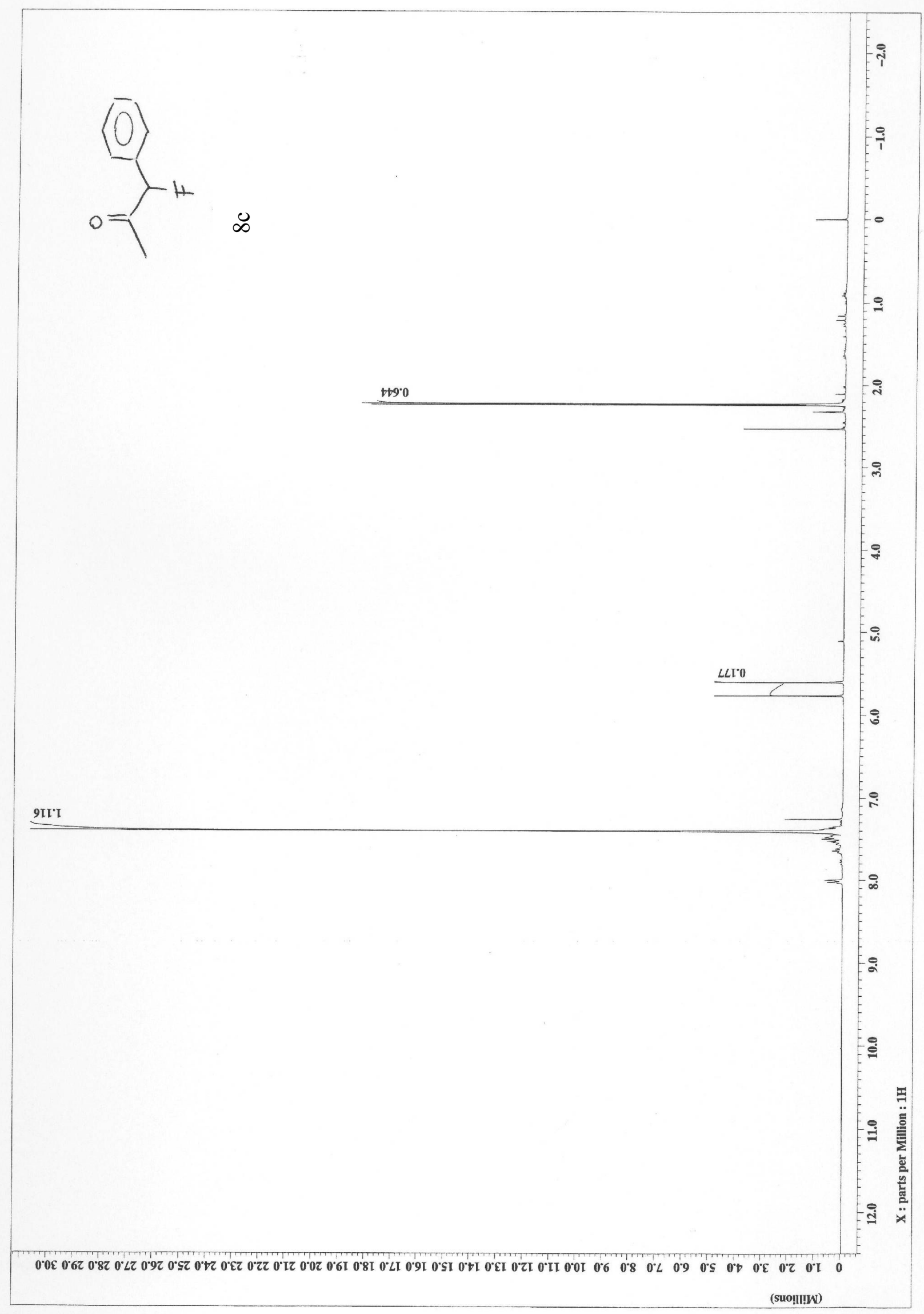




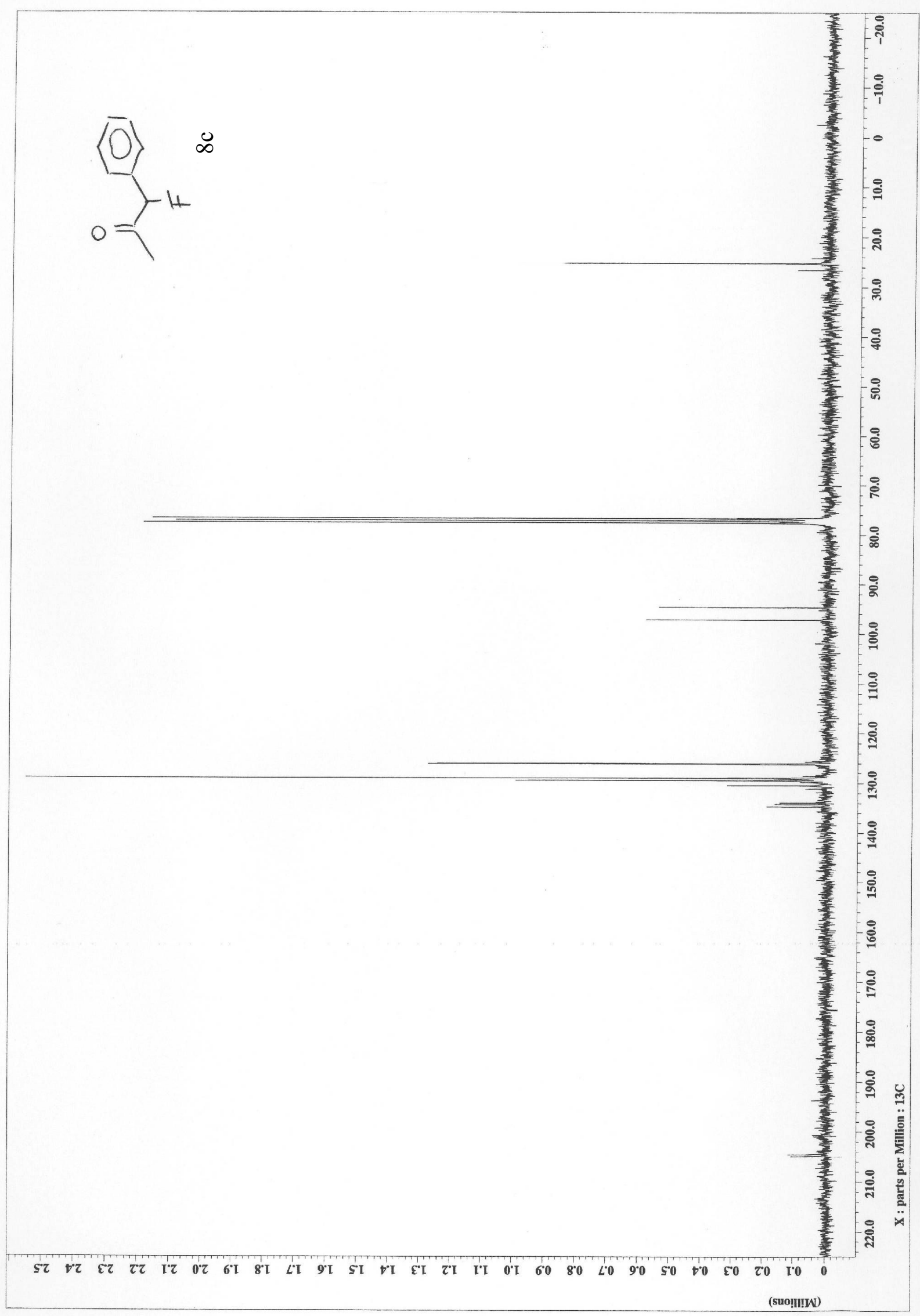




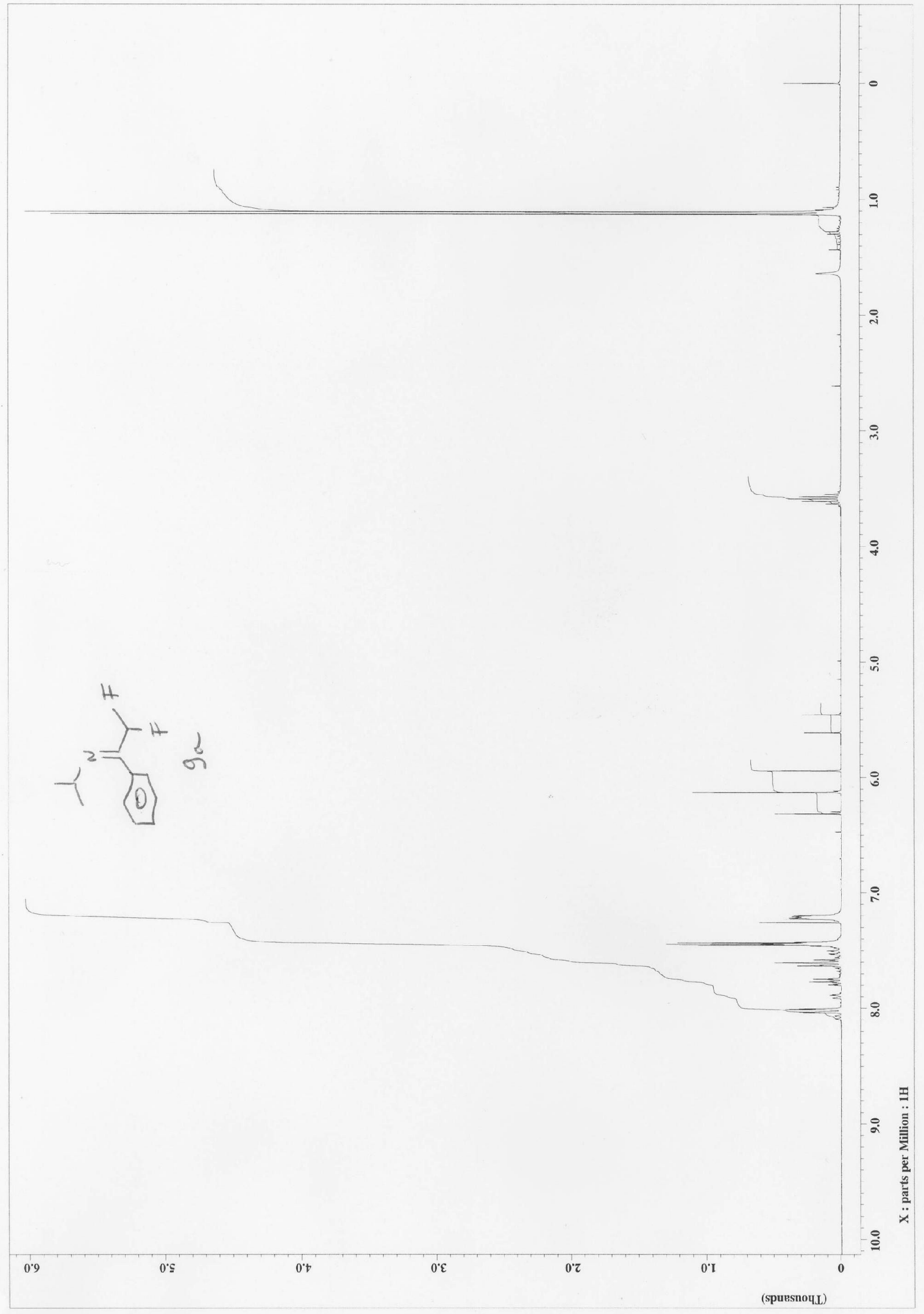









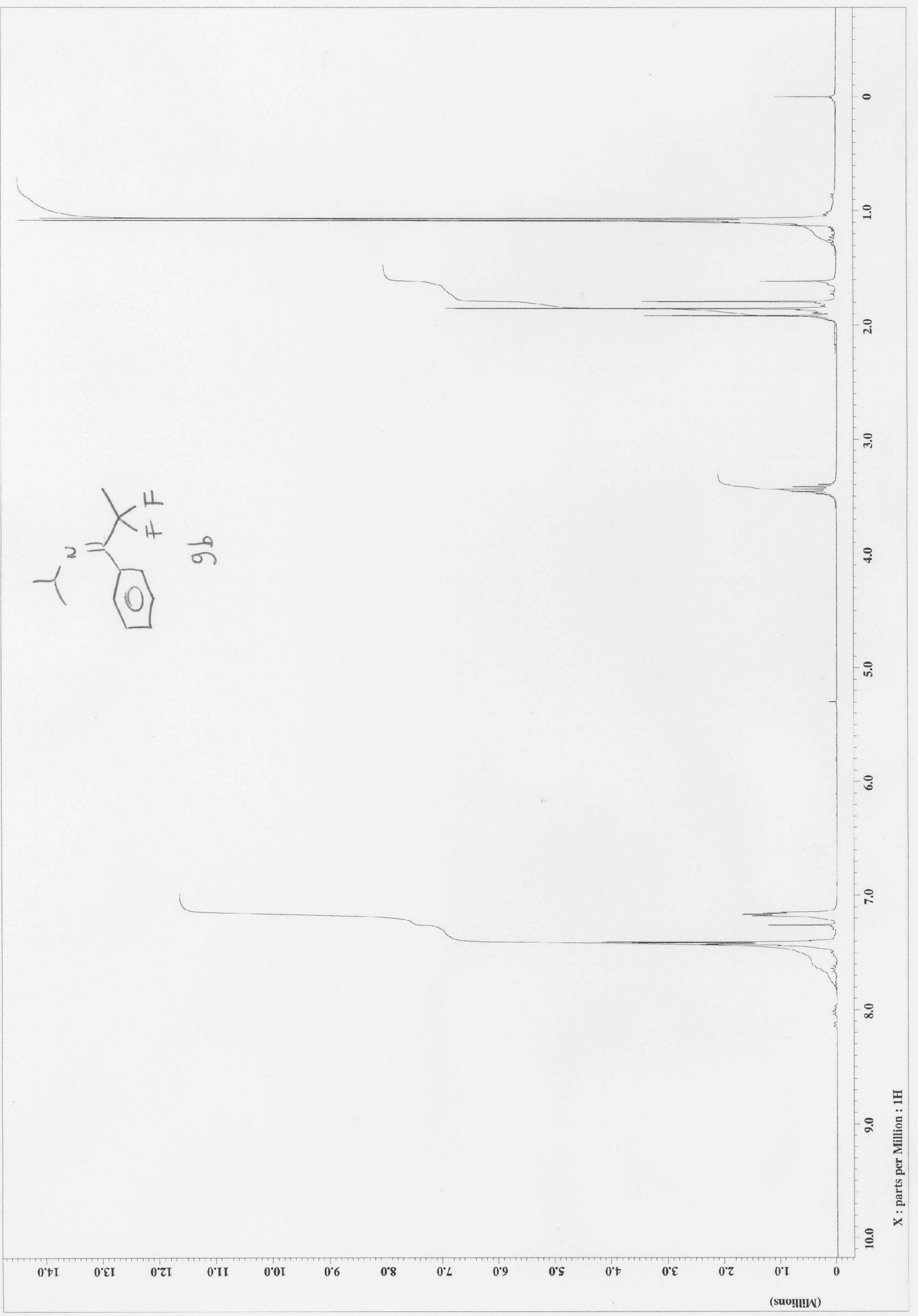




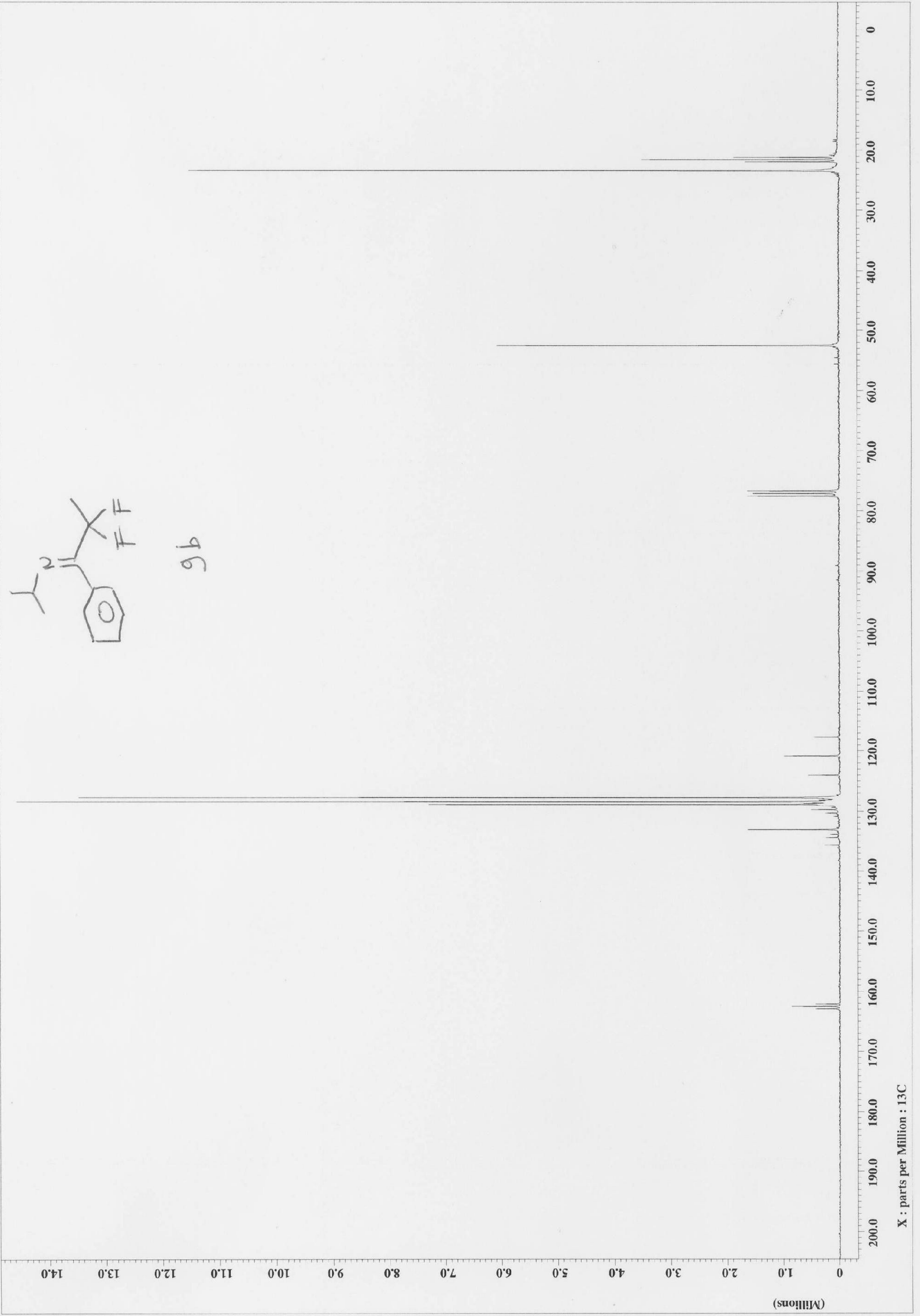




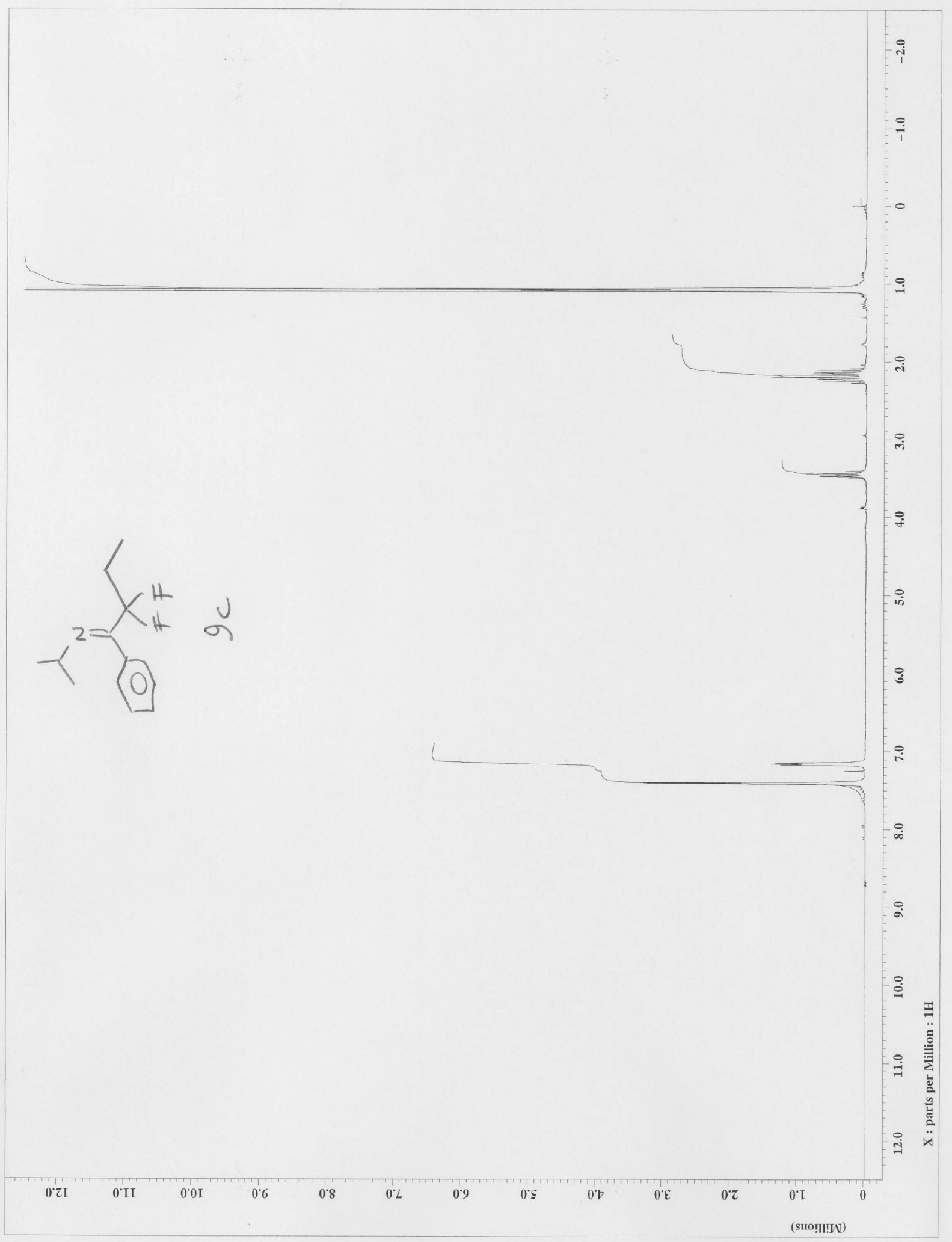




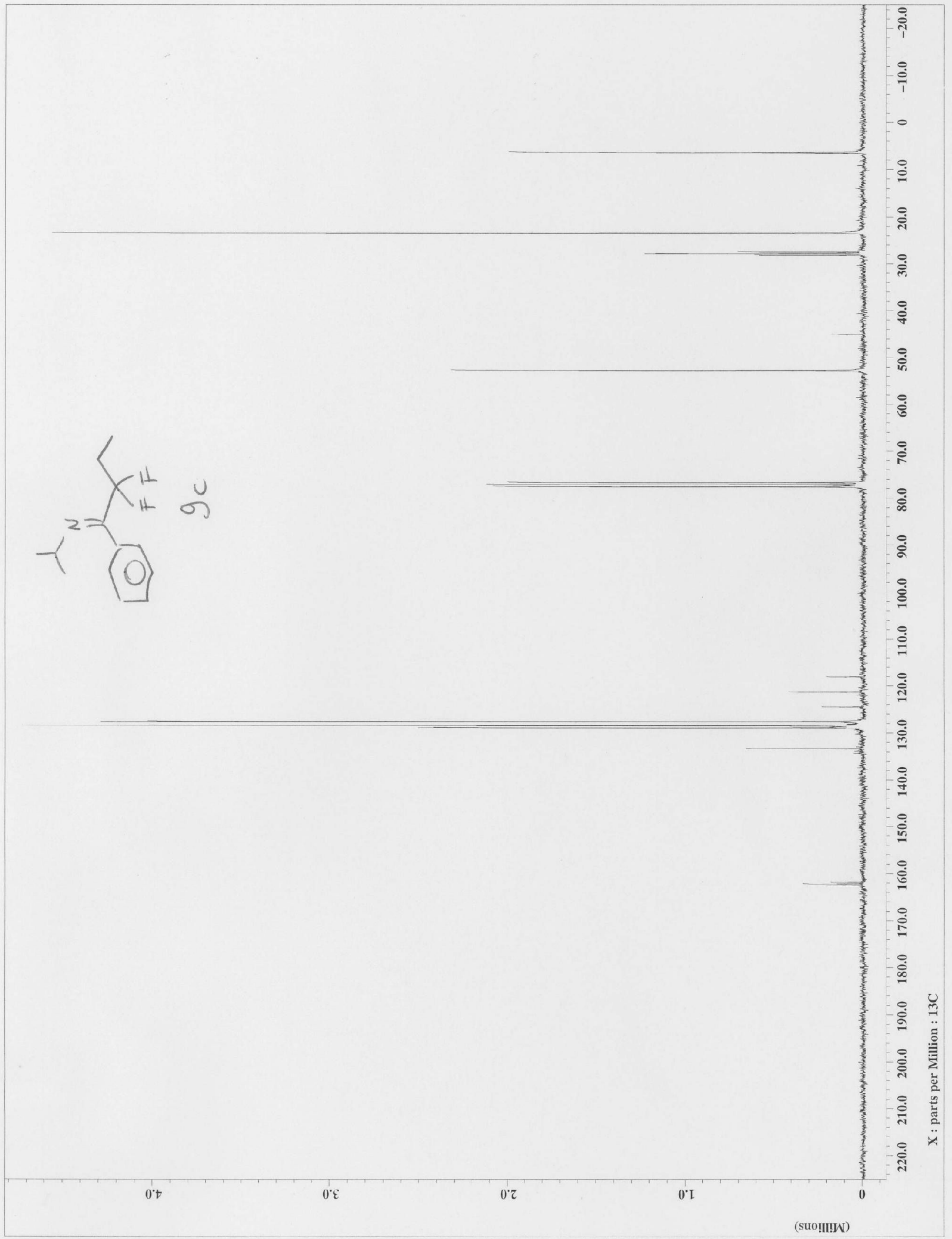




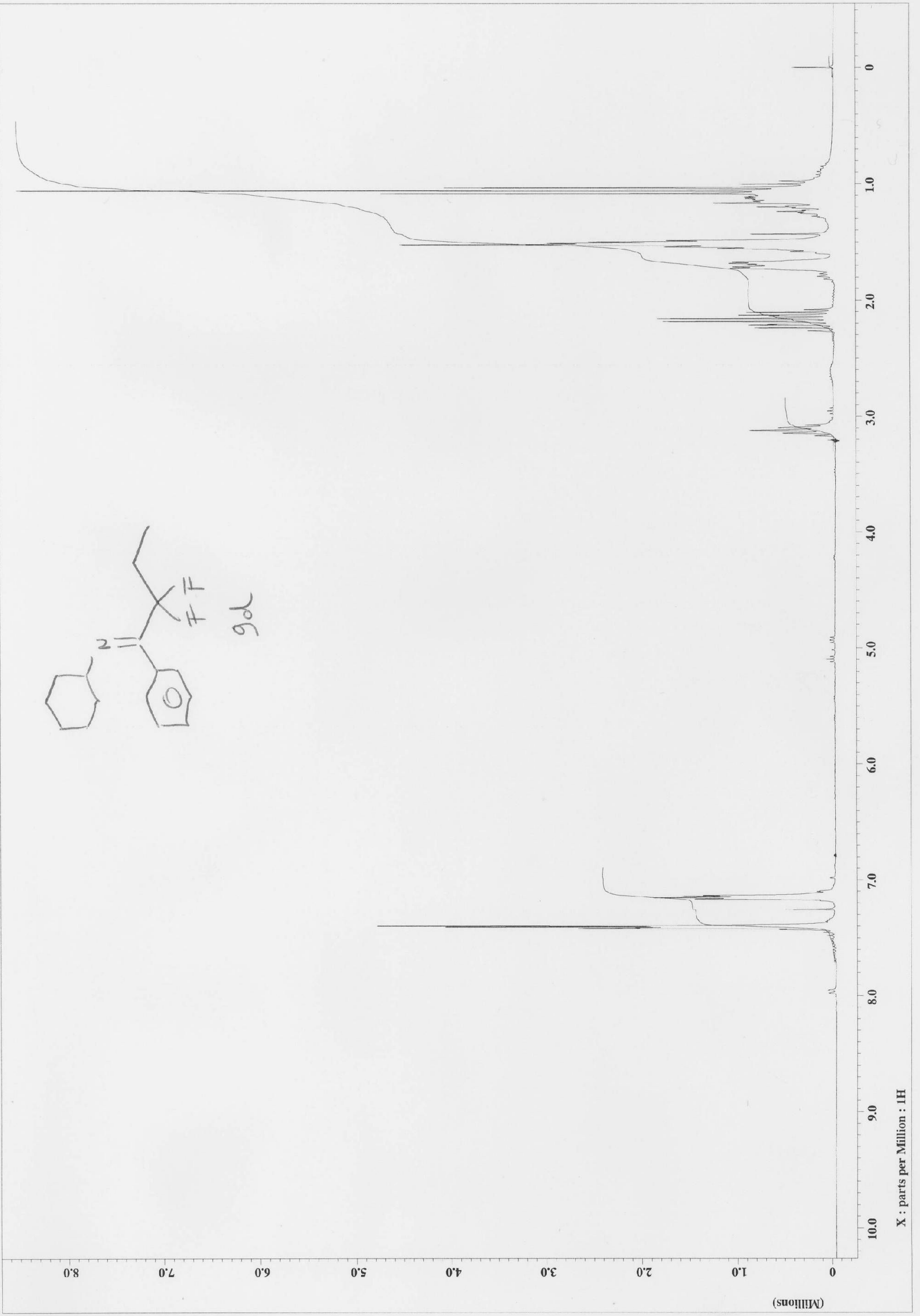




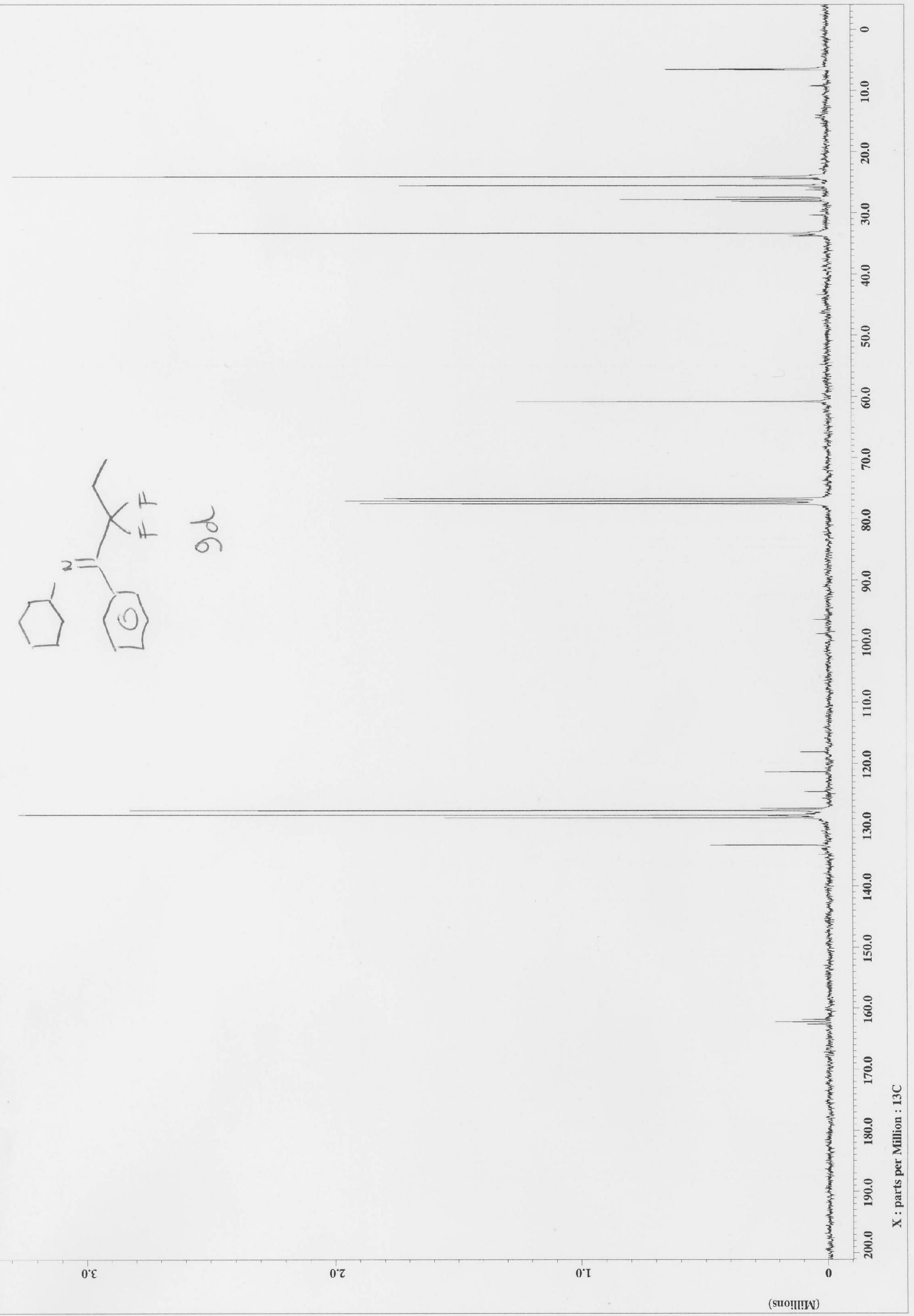




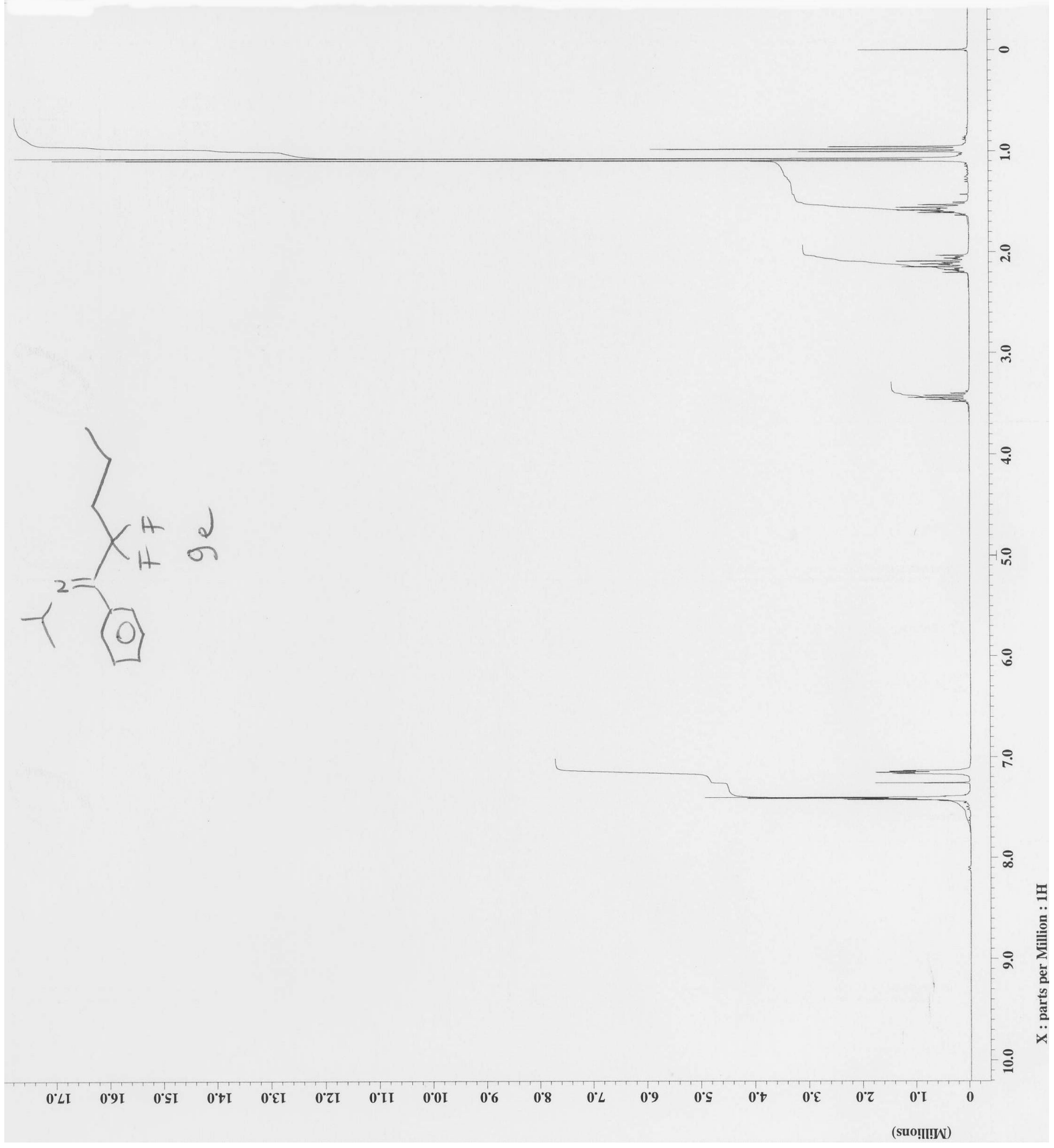




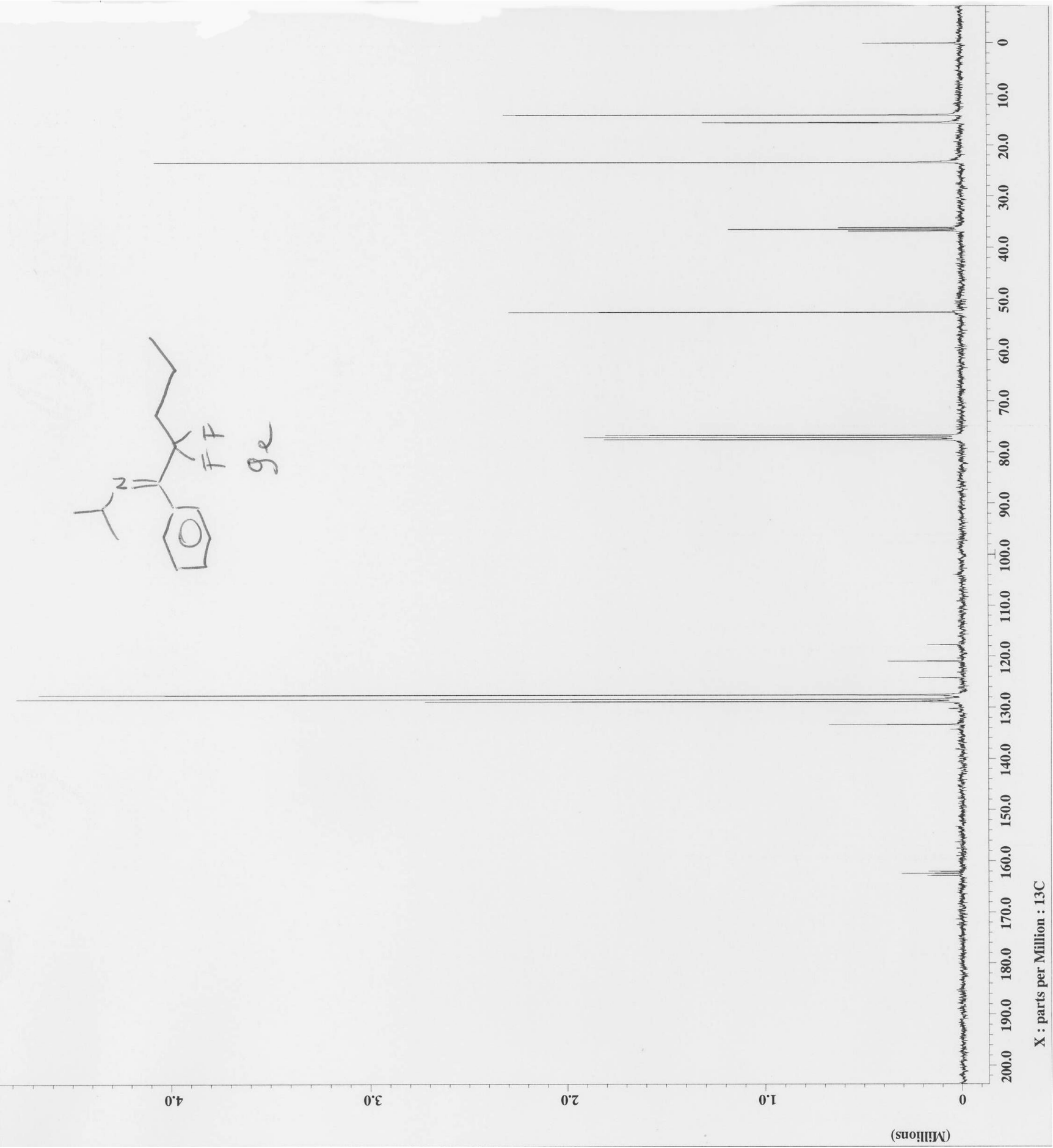




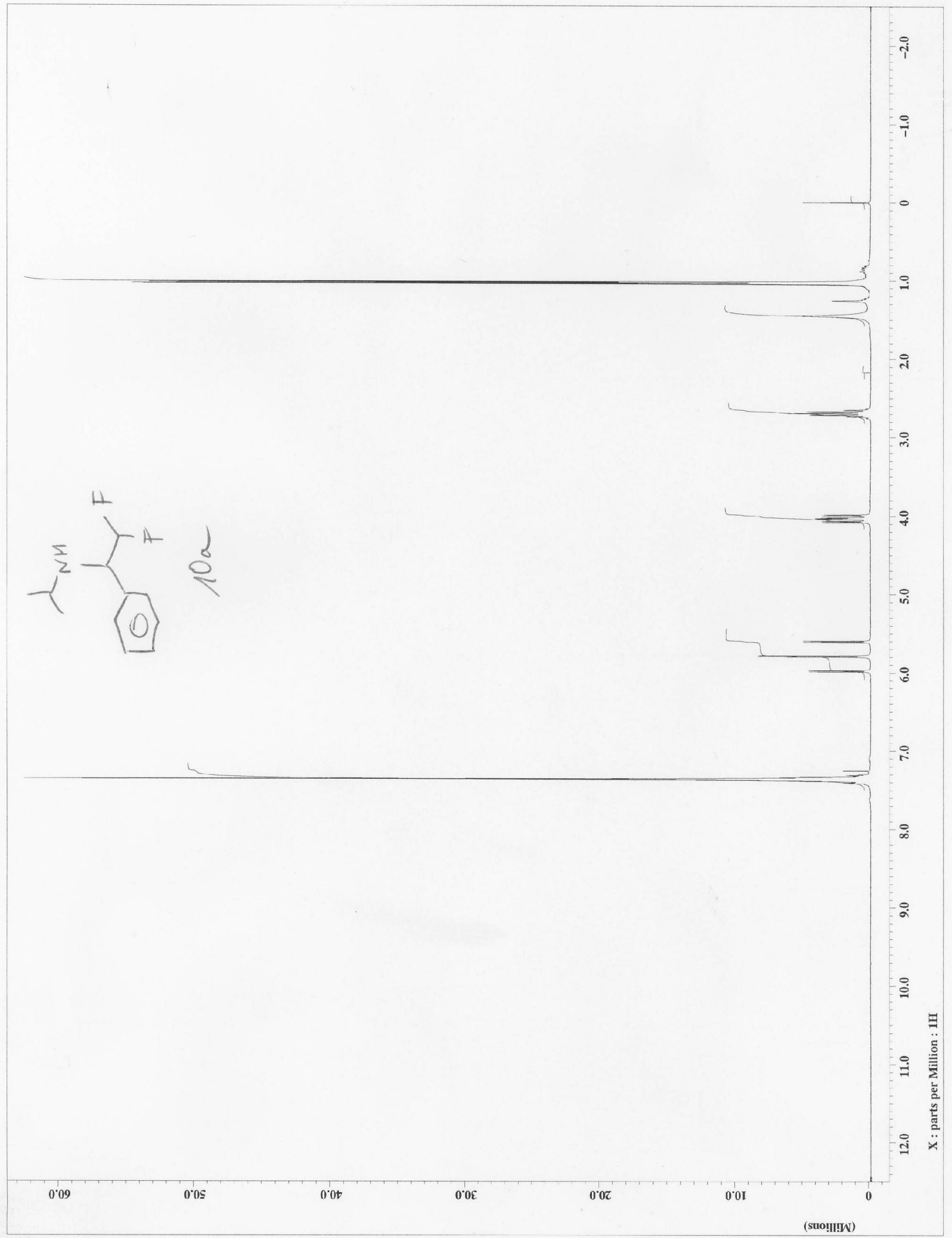




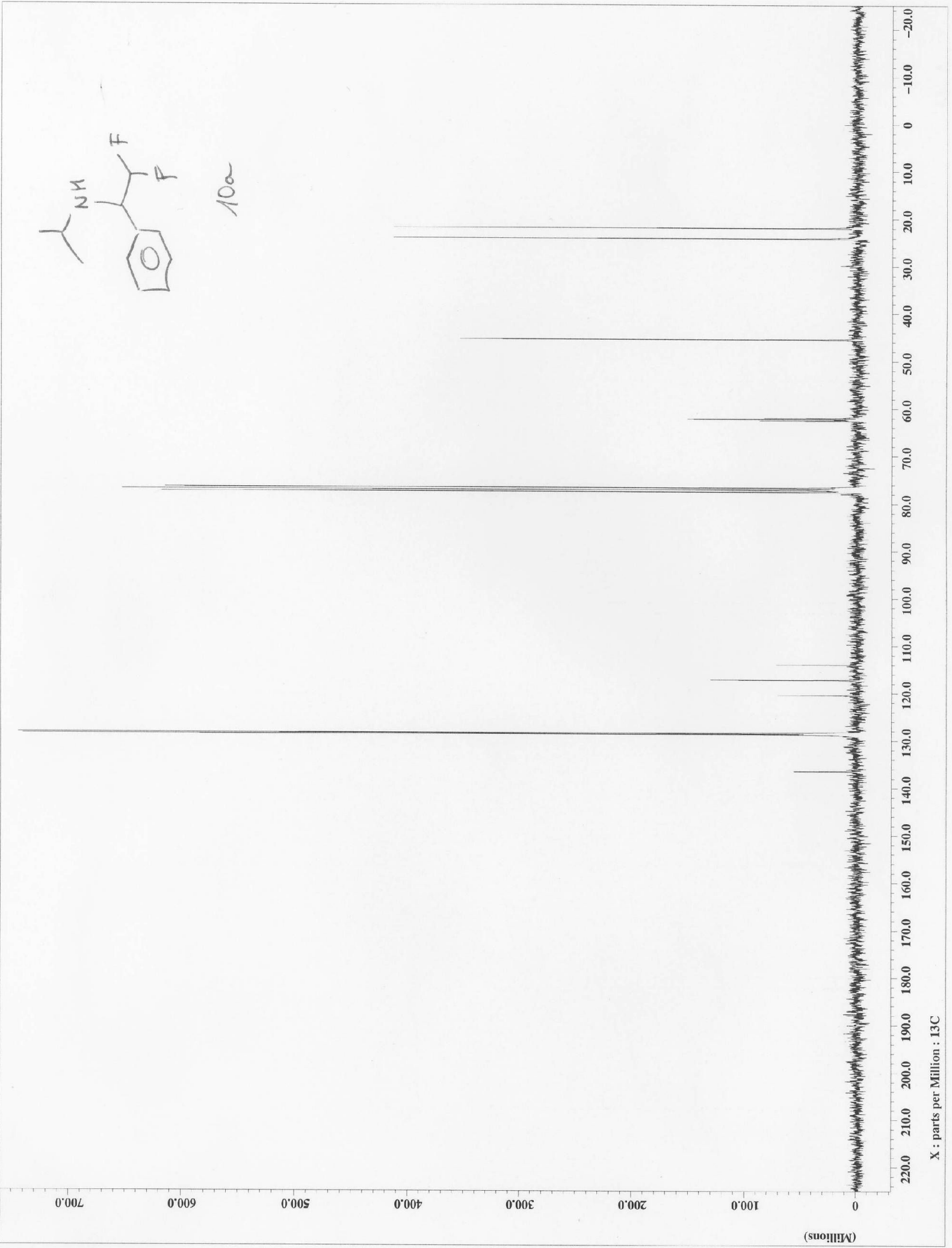




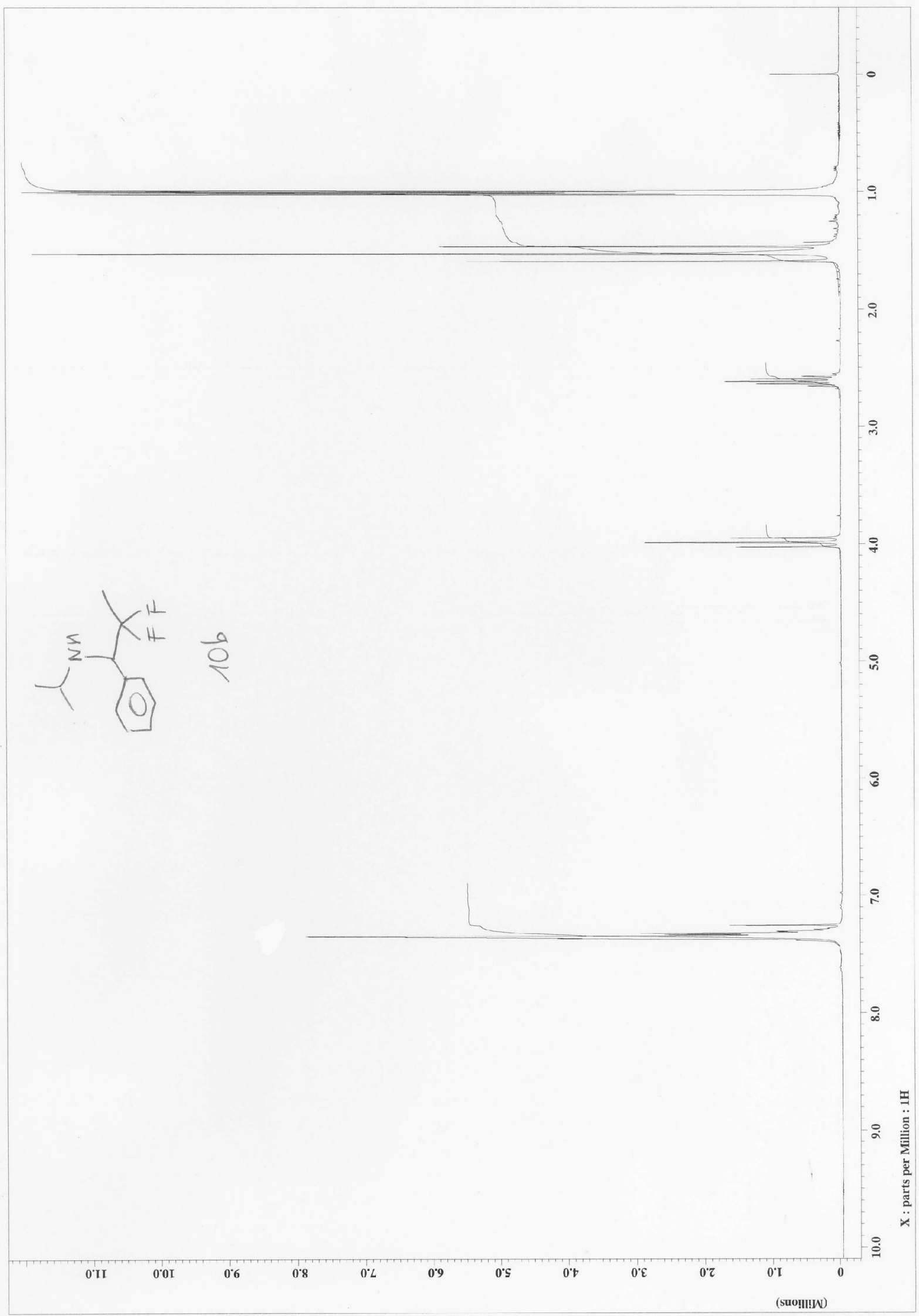




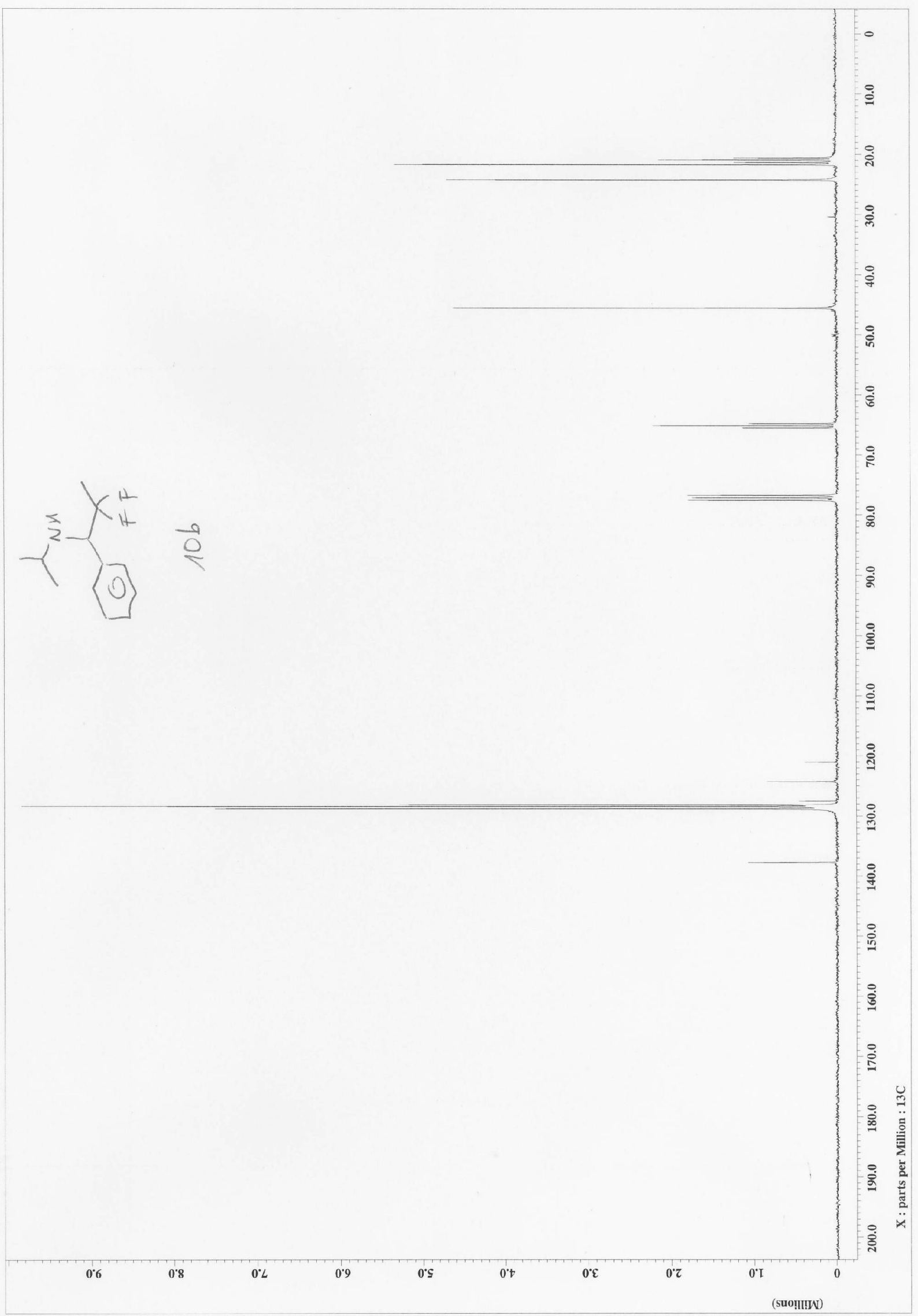









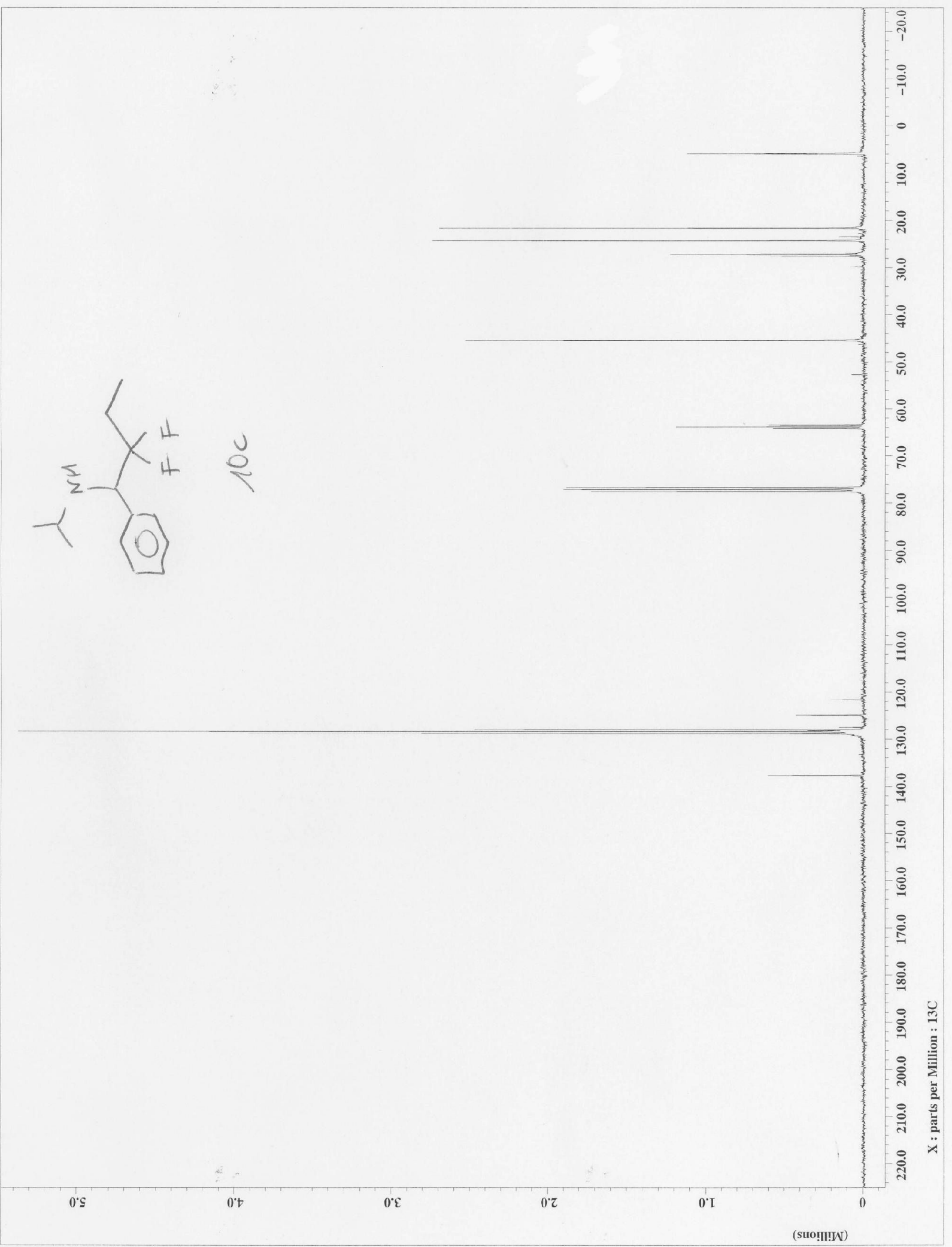




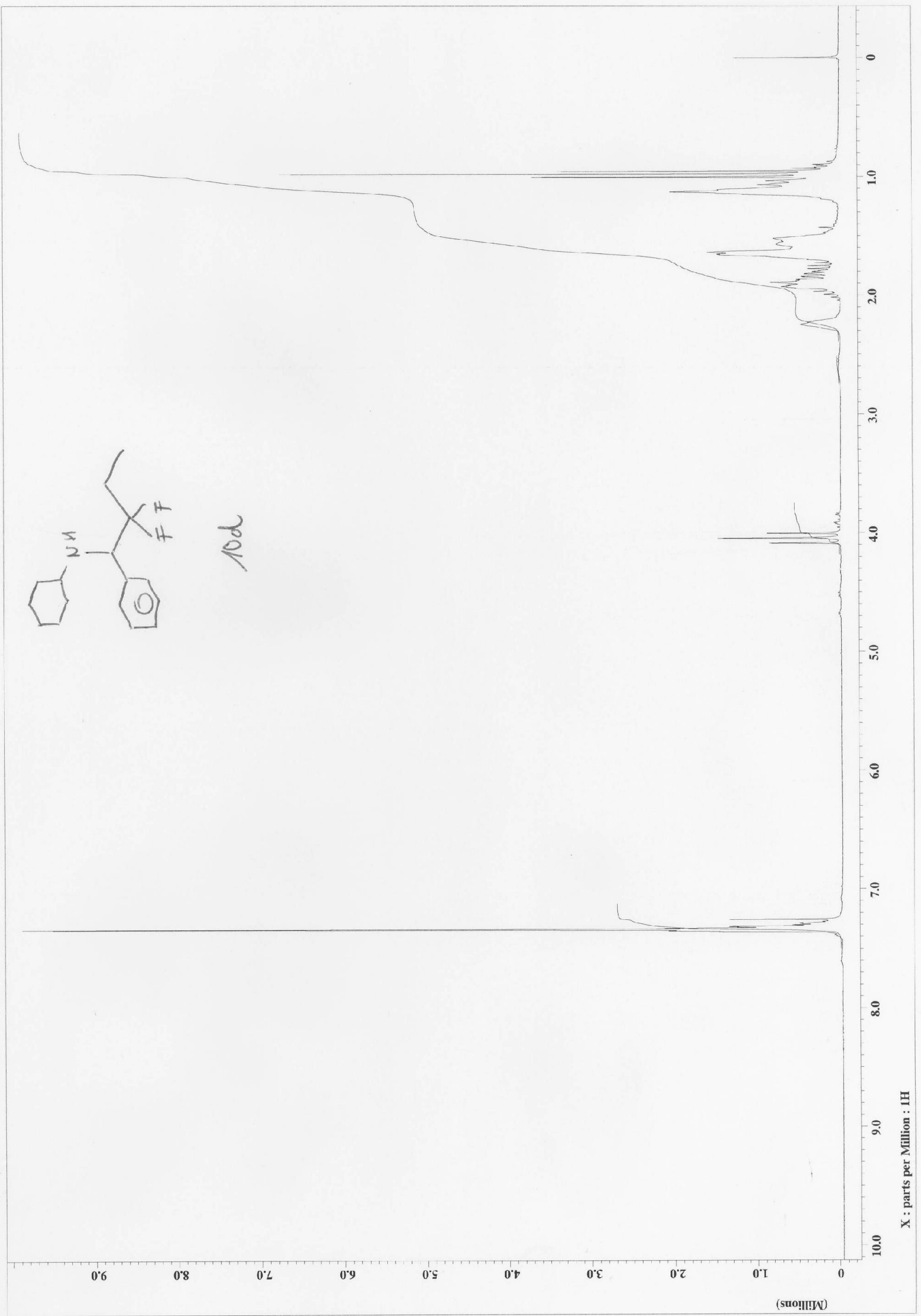




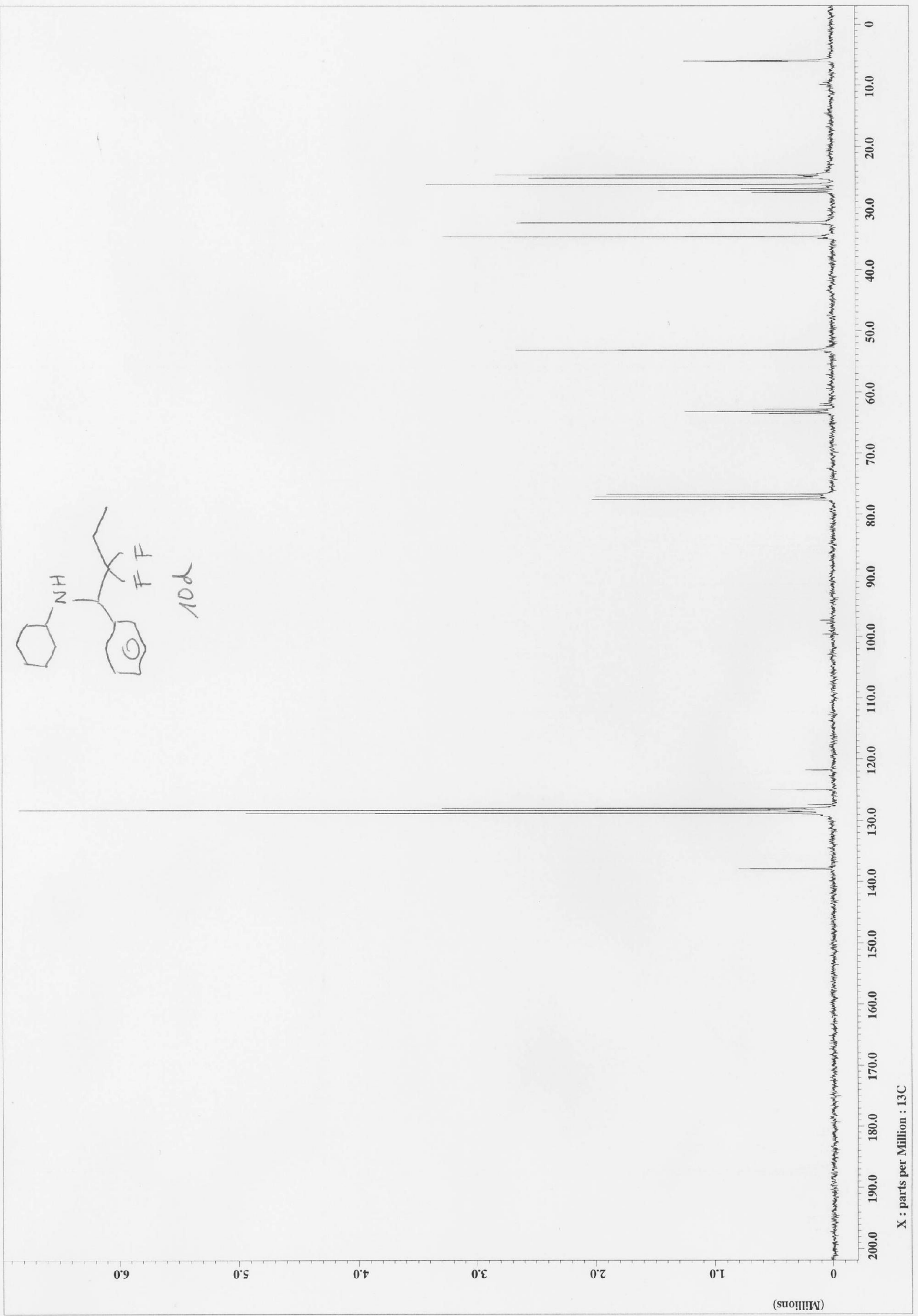




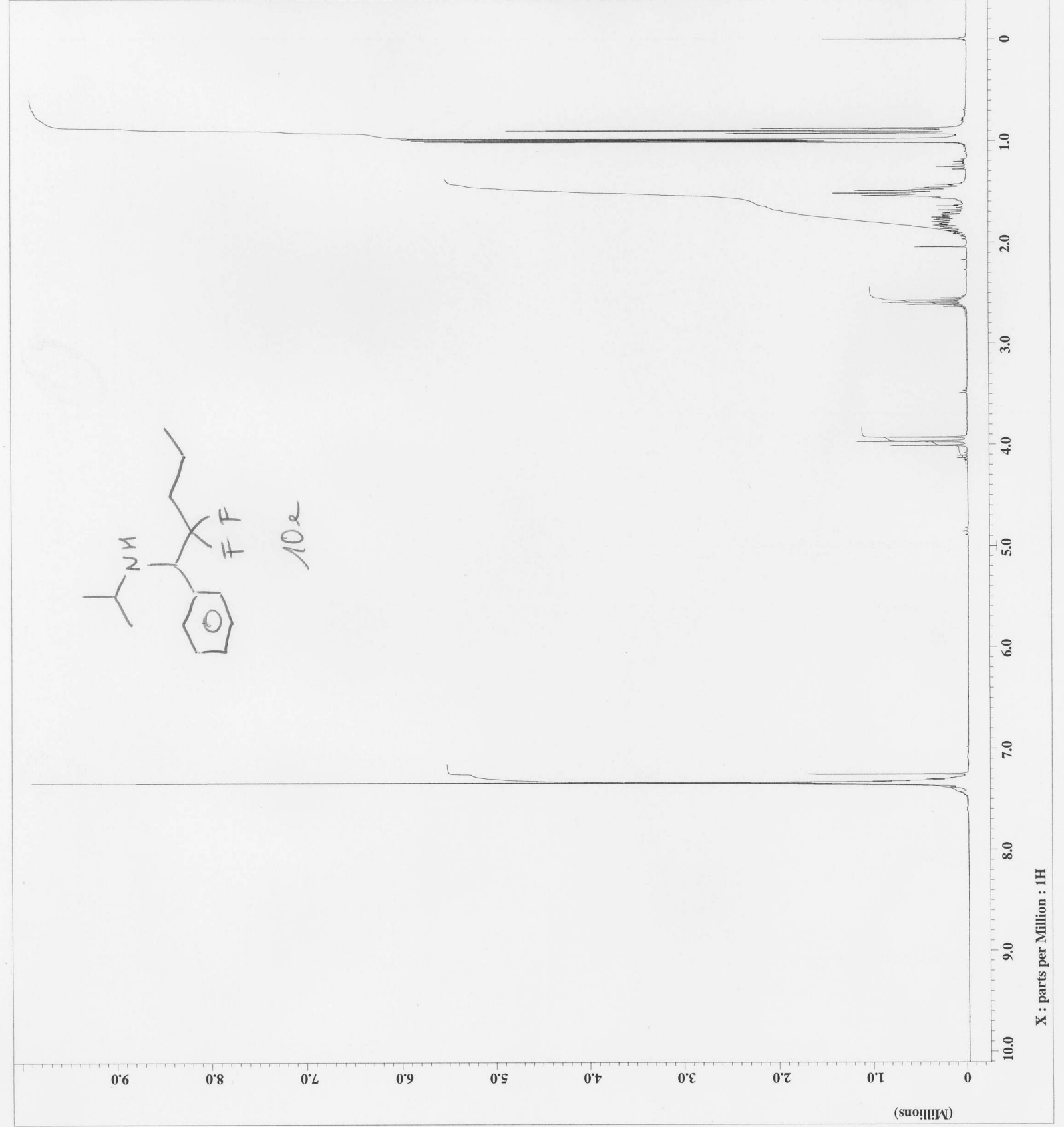


S-73

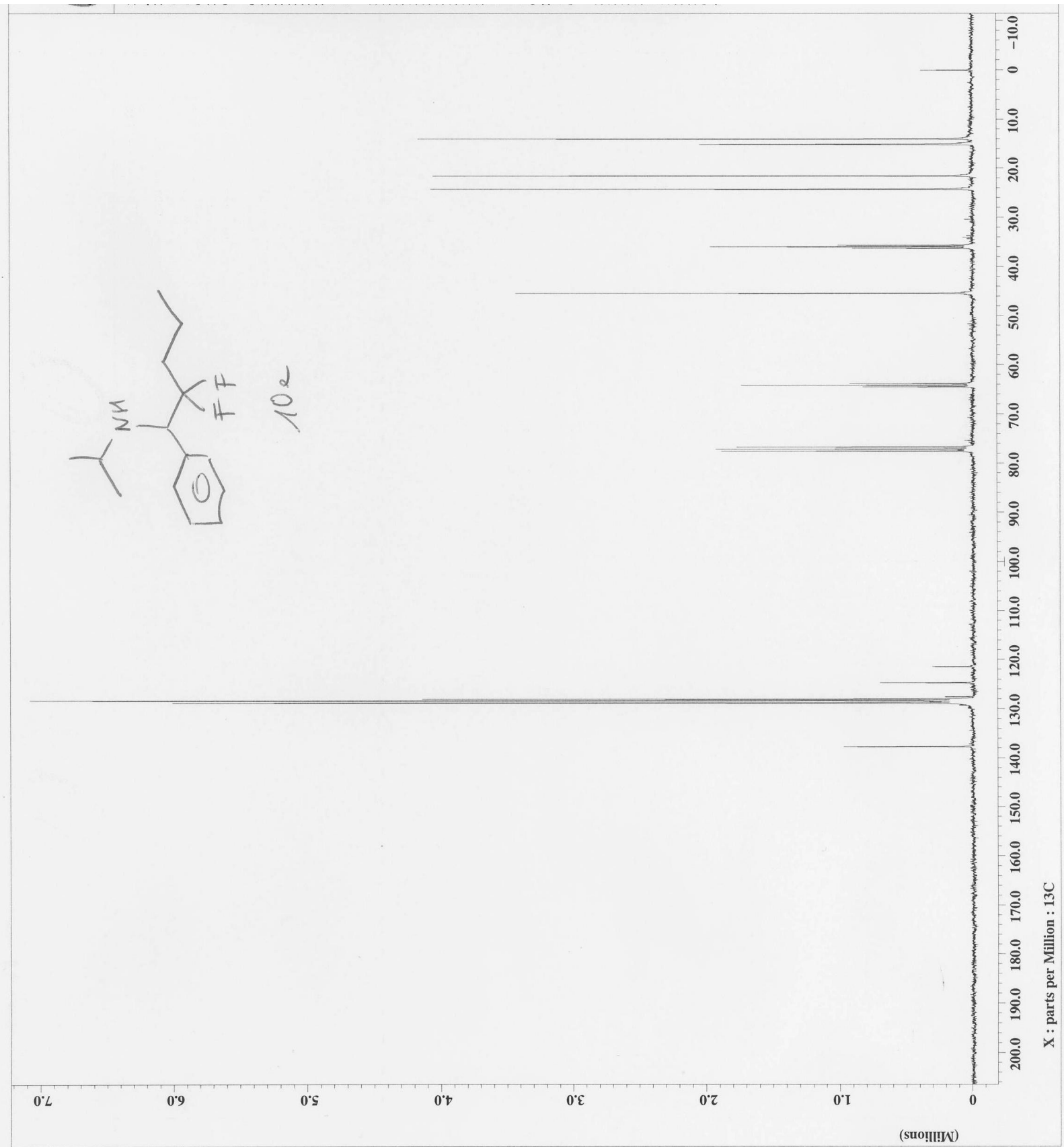

